\title{
Youth Aspirations and Expectations of Economic Recovery of Jammu and Kashmir in India after Abrogation of Article 370
}

\author{
Sofia Aslam ${ }^{\circledR}$, Falendra Kumar Sudan* \\ Department of Economics, University of Jammu, Jammu, India \\ Email: fk_sud@rediffmail.com
}

Received: 8 June 2021; Revised: 3 September 2021; Accepted: 4 September 2021

\begin{abstract}
The paper intends to explore the perceptions of the youth regarding the economic recovery of Jammu and Kashmir in India after the abrogation of Article 370, and to draw the policy implications for the speedier economic recovery of conflict-ridden regions. The study has been confined to the Jammu region and focused on four selected districts of the Jammu region viz Jammu, Kathua, Rajouri, and Poonch. A cluster sampling technique has been used to select respondents from the selected districts. The study has been restricted to the educated youth of selected districts as they represent the hope for the future and they can better interpret economic recovery. The primary data has been collected through a questionnaire survey of the youth of selected districts. A total of 120 educated youth (graduates, postgraduates, research degree holders belonging to different religious groups viz. Hindus and Muslims: 40 each, and Christians and Sikhs: 20 each) have been selected based on their approximate proportional representation in the total population. Data has been analyzed using simple descriptive statistics. The paper reveals that economic recovery requires significant development of businesses and local economies. Young population is the heartbeat and energetic force for economic development. Therefore, their participation in economic recovery is vital to give right direction to the economy specifically since the revocation of the special status of Jammu and Kashmir. The developmental aspirations of the youth focused on agriculture reforms, investment in manufacturing, transportation, health, education, infrastructure, banking, and transparency in all development planning and implementation. Youth education and training and self-employment including the development of tourism, horticulture and handicrafts sectors were prioritized for economic recovery post-abrogation of Article 370. Addressing persistently high unemployment rate in Jammu and Kashmir has been ranked as one of the top priority areas for economic recovery. There is a need to rejuvenate the sick industrial units for creating sustained employment opportunities for youth. Tourism, handicrafts and horticulture sectors should be developed to reduce poverty, promote exports and generate employment for the youth through substantial funding from the Central government and private sector.
\end{abstract}

Keywords: conflict, economic recovery, youth, expectations, Jammu and Kashmir, India

JEL Codes: A14, D70, D74, D84, F43, J13

Copyright (C2021 Falendra Kumar Sudan, et al.

DOI: https://doi.org/10.37256/redr.222021980

This is an open-access article distributed under a CC BY license

(Creative Commons Attribution 4.0 International License)

https://creativecommons.org/licenses/by/4.0/ 


\section{Introduction}

Economic recovery refers to the process of recovery of businesses and local economies after the disaster (Chang \& Rose, 2012). Economic recovery is the rebuilding of the socioeconomic framework and reconstruction of the enabling conditions for the functioning economy (Rose \& Krausmann, 2013). It is a process of return from insecurity and inconsistency to a normal development path through a self-sustaining process of economic governance. Financial recovery and creating jobs are the enabling priorities to start reconstruction (Jomo \& Chowdhury, 2020). Public expenditure can adequately address horizontal inequalities and promote comprehensive development. Private investment fosters public investment and promotes economic development. Economic reforms should be compatible with the political and economic realities of the country (Nayyar, 2008).

Successful economic recovery requires robust governance for peace building. In the broader perspective, economic recovery means attainment of higher socioeconomic well-being, including food security, public health, shelter, educational systems, a social safety net for all citizens, reconstruction of physical and social infrastructure, employment generation, well regulated markets, legal and regulatory reforms, robust trade and investment, and transparent banking and financial institutions (Amsami, 2019). India can tap its human and natural resource potential through better institutions to facilitate poverty reduction and ameliorating youth employment. Economic performance can be restored by reconstruction of infrastructure, accessibility of capital markets, labor market, management of public finance, and availability of government services (Chang et al., 2012).

Young people are an important human resource and energetic force for development. Youth participation in economic recovery is vital for all round development of the economy including industrial development and long-term stability. Youth is considered as an important resource for economic growth and brings huge cost-effective benefits. Youth of Jammu and Kashmir in India also aspires to make a positive contribution towards the economic recovery of conflict-ridden society but their ability to do so is hampered by the ongoing political insecurity and educational and employment challenges.

Jammu and Kashmir, which has been a subject of conflict between India, China and Pakistan since the lines of partition were drawn. Later on, Jammu and Kashmir was given the unique status of Article 370 drafted in Part 21 of the Constitution of India, which granted Jammu and Kashmir powers to make laws, except for defense, external affairs and, communications. It was stated that the provisions concerning Article 370 are temporary, not permanent. In 1990, the rapid surge in militancy in the Kashmir valley forced the Kashmiri Pandits (a minority Hindu community) to migrate in large numbers to Jammu and other parts of the country. Over the period, numerous armed conflicts occur in Jammu and Kashmir, which deteriorated the psychological and economic conditions of the masses. On $5^{\text {th }}$ August 2019, the Government of India renounced the Article 370 of the Constitution of India and on $31^{\text {st }}$ October 2019, Jammu and Kashmir State was divided into the two Union Territories viz. the Union Territory of Jammu and Kashmir and Union Territory of Ladakh for speedier economic development and better governance.

A number of studies have been carried out on the economic recovery of different countries even in India including Jammu and Kashmir. However, the studies conducted on the economy of Jammu and Kashmir have been conducted before the abrogation of Article 370. With the scrapping of Article 370, there is a need to know the perceptions of stakeholders regarding economic recovery in Jammu and Kashmir. There is also a need to know what policies the government should implement in the future to improve the condition of Jammu and Kashmir economy. Besides, no extant studies have been undertaken to know the youth's perceptions and expectations related to the economic recovery in Jammu and Kashmir after abrogation of Article 370. Therefore, the present study is an attempt in this direction and fills the knowledge gaps on the youth perceptions and expectations linked to the economic recovery in Jammu and Kashmir in the aftermath of Article 370. Against the above backdrop, the paper intends to explore the aspirations and expectations of youth on economic recovery of Jammu and Kashmir in the aftermath of the abrogation of Article 370 with a goal to offer policy implications. The study has been confined to four districts of Jammu region and used cluster sampling technique to select the sample population and collection of primary data and information. Simple descriptive statistics have been used to analyze the data.The study reveals that economic recovery in aftermath of abrogation of the Article 370 needs all round development of the conflict-ridden economy of Jammu and Kashmir through active participation of all the stakeholders including the youth. Youth's developmental aspirations should be given special attention in holistic development of all sectors. Transparency and good governance should be the key consideration in economic recovery policies and programs. Youth employment should be the cornerstone of all developmental 
intervention in the economic recovery of Jammu and Kashmir.

\section{Review of literature}

Economic recovery is a process of socioeconomic transformation (Strohmaier et al., 2019; UN, 2020). Economic recovery needs a better understanding of indigenous drivers (UNDP, 2008), which provide the most effective ways for the post-war rehabilitation efforts (ILO, 2010). Robust governance and institutions are essential for economic recovery (Liu et al., 2018). Economic recovery requires substantial public expenditure (Mills, 1934; Zouhar et al., 2021), more effective development strategies (Kedia et al., 2020), social sector programs, industrial development and diversification (Thanoon et al., 2014), increasing the aggregate demand (Summers, 2010), rejuvenating the private sectors, and broader participation in growth (ILO, 2014).

In the recent past, economic growth remained low in Jammu and Kashmir due to ongoing conflict. The economy moves towards stagnation and common people suffer the most (Mahapatra \& Shekhawat, 2008; Singh, 2017). The Article 370 was the root cause of corruption, state alienation, terrorism, and poverty (Shah, 2018). The abrogation of Article 370 will help to stabilize Jammu and Kashmir economy by integrating into India through economic recovery and reducing poverty.

Youth unemployment is pervasive worldwide due to lack of skills, work experience, job search abilities, and financial resources (Moore, 2019). India is no exception in rising youth unemployment. Similarly, youth unemployment is alarming in Jammu and Kashmir (Nengroo, 2015) due to changing occupational patterns from agriculture to the service sector without taking into account the development of the secondary sector (Khan, 2013). Inadequate development of the private sector forced the educated unemployed youth towards the government jobs. Therefore, the government should take necessary steps to increase the employment opportunities for youth in Jammu and Kashmir (Qadri \& Kasab, 2017). The abrogation of Article 370 will enable the private investors to invest in industrial sectors, which will increase youth employment in Jammu and Kashmir.

Health facilities are much better in Jammu and Kashmir than the rest of the Indian States (Mir \& Bhat, 2018). Even then, the majority of poor people faced health infrastructure constraints due to lack of private investment due to restrictions of Article 370. Education promotes human capital development and overall economic development (Sreenivasul, 2013). Inadequate educational development mars development process in Jammu and Kashmir due to poor infrastructure (Shah, 2018). The Article 370 restricts the youth of Jammu and Kashmir from effective career competition.

Development of physical infrastructure is vital for economic development. Infrastructure development contributed significantly in the reduction of economic disparities and abysmal poverty in Jammu and Kashmir. Strong economic growth requires robust infrastructure (Gachunga \& Kuso, 2019). Violent conflict in Jammu and Kashmir has caused serious damage to the existing infrastructure, which needs to be rebuilt to revive the economy of Jammu and Kashmir (Mahapatra \& Shekhawat, 2008). Revocation of Article 370 will open opportunities for growth and development. Corruption impacts economic growth directly or indirectly and reduces human capital and private investment in Jammu and Kashmir (Dimant \& Tosoto, 2018; Bhattacharyya \& Jha, 2013; Mir, 2019).

Tourism plays a significant role in economic development of India (Khatik \& Nag, 2012) and Jammu and Kashmir (Mir, 2014). The scrapping of Article 370 presents an immense opportunity for Indians' travel and tourism sector in Jammu and Kashmir as the outsiders will now be able to make real estate investments. Indian handicraft industry is labor-intensive and contributed significantly to exports (Bhat \& Yadav, 2016). Jammu and Kashmir rely largely on the handicrafts sector, which brings enormous foreign exchange earnings (Bhat, 2019) and creates wealth and jobs (Bhat, 2017).

Investment in horticulture sector contributes significantly to production and productivity and improves the living standard of people and generates employment (Islam \& Shrivastava, 2017). Horticulture industry in Jammu and Kashmir has huge potential for employment and revenue generation, and poverty reduction (Darzi, 2016). Horticulture sector in Jammu and Kashmir faces severe challenges like debilitated infrastructure, weak supply chain, poor marketing strategy, problem of wastage, and higher costs (Lone, 2014). The revocation of Article 370 will boost agriculture including horticulture development in all three regions viz. Jammu, Kashmir, and Ladakh.

Investment is significant for economic recovery, which increases foreign investments (Breuer et al., 2010; Erden \& Holcombe, 2006) and public investment to stimulate private investment. After the aftermath of Article 370 in Jammu and Kashmir, the venture opportunities will increase. Economic recovery in Jammu and Kashmir is possible by allowing 
the private sectors to invest in different sectors like tourism, transportation, hydropower generation, dairy products, sericulture, horticulture, information technology, food security, handicrafts, and agriculture by harmonious policy framework (Upadhyay, 2019).

Macroeconomic policies play an essential role in economic growth (Perkins, 1979) by rebalancing the economy (Mohanty, 1997). Credit is significant for the financial advancement of the country (Bhosale, 2014). Commercial banks substantially contribute to industrial development. Economic growth of India generally relies upon the banking sector (Rehman, 2016). Banking sector contributes immensely to the economy of Jammu and Kashmir (Abrol, 2014).

Violence results in lack of employment opportunities and a poor educational system (Keen, 2003). Youth bulges led to rising anxieties and vulnerabilities to violent conflict involving youth in terrorism. Jammu and Kashmir is one of the most conflict-ridden areas in the world. The development scenario of Jammu and Kashmir has been severely affected by the continued violence (Bhat, 2019), which require successful implementation of developmental policies for speedier economic recovery (Mahapatra \& Shekhawat, 2008).

The literature reviewed clearly makes it evident that no study exists to explore the perception, attitudes and aspirations of youth in Jammu and Kashmir specifically after the abrogation of Article 370. With the scrapping of Article 370 , there is a need to understand the youth's perceptions regarding economic recovery of Jammu and Kashmir with a goal to know what policies the government should implement in the future to improve the economic condition and speedier economic recovery.

\section{A framework of youth aspirations and economic recovery}

Aspirations refer to the capacity to steer social life and support desires, preferences, and choices to achieve the intended goals in a society (Appadurai, 2004). Aspirations can be ex-post or ex-ante. Ex-post aspirations are futureoriented and can be fulfilled in near future. Ex-ante aspirations are linked to instantaneous fulfillment of goals. Aspirations are the targets to be achieved with investment of time, energy or capital. In this study, youth aspiration is different from inoperative fantasies and desires. The likelihood of aspirations failure is termed as aspiration gap (Ray, 2006). Aspirations failure may be attributed to a behavioral bias (Dalton et al., 2016). Aspirations can be realized through concerted efforts for positive development outcomes (Lybbert \& Wydick, 2018). Successful aspirations are realized through a genuine goal for the future, initiation of required steps to achieve the target and chalk out the road map to fulfill the goal (Snyder, 2002). Aspirations based on achievable goals, situations and endeavors aligned to expectations can bring victorious results (Dalton et al., 2016). Therefore, aspirations are based on preferences, while expectations are linked to practical perceptions. Figure 1 shows the framework of youth aspirations and economic recovery.

In this study, aspiration refers to the ex-post aspirations of educated youth from the government for economic revival in the aftermath of abrogation of the Article 370 of the Constitution of India. It also refers to follow the aspired goals and to work actively to achieve the goals by the younger generation. Aspirations are linked to ones actions and interactions to achieve the targeted goals. Aspirations can determine educational and occupational achievements. Job aspirations can impel the preference for education, employment goals, and salaries including lifetime outcomes. In the context of abrogation of the Article 370, youth aspirations shape how economic recovery is achieved through government interventions. It helps in understanding how aspirations affect the lives of youth and why aspirations are at the center of economic recovery in the aftermath of the Article 370. Therefore, this study investigates youth aspirations vis-à-vis economic recovery in Jammu and Kashmir in post-abrogation of the Article 370.

Very high or very low aspirations dampen enthusiasm to achieve the goals. Likewise, aspirations matched with expectations can bring higher outcomes. Therefore, the economic development policies and governance in the postabrogation of 370 phase should be built in a manner compatible with aid to the youth realize their aspirations of speedier economic recovery. This can also provide a potential roadmap to achieve their career goals and the development efforts can efficiently activate the inspiring power of youth aspirations.

The capacity to aspire depends on socioeconomic background of individuals. For instance, education silhouettes how youth performs in society. Education and learning establish how youth behave and what they judge to be achievable. Education can develop youth's capability to succeed and achieve the targets. Youth's self-efficacy significantly determines their aspirations (Sen, 1985). Capabilities can significantly influence the real opportunities 
(Robeyns, 2016), which also depends on social situations (Drèze \& Sen, 2002) and an individual's own experiences (Dalton et al., 2016). Therefore, youth education and learning and their capabilities to contribute to economic recovery shape future policies in post-abrogation of the Article 370.

Aspirations can build the future well-being of youth to meet their career expectations based on development policies to be pursued by the government. If youth perceive that they have the capability to bring significant transformation in their lives through concerted effort (Lybbert \& Wydick, 2018; Dalton et al., 2016), they can chart the roadmap to achieve the goals at their own (Ray, 2006; Appadurai, 2004; Bandura, 1977) or through government policies (Lybbert \& Wydick, 2018). Therefore, youth can most likely to tap the opportunities offered by the Abrogation of the Article 370 through government policies.

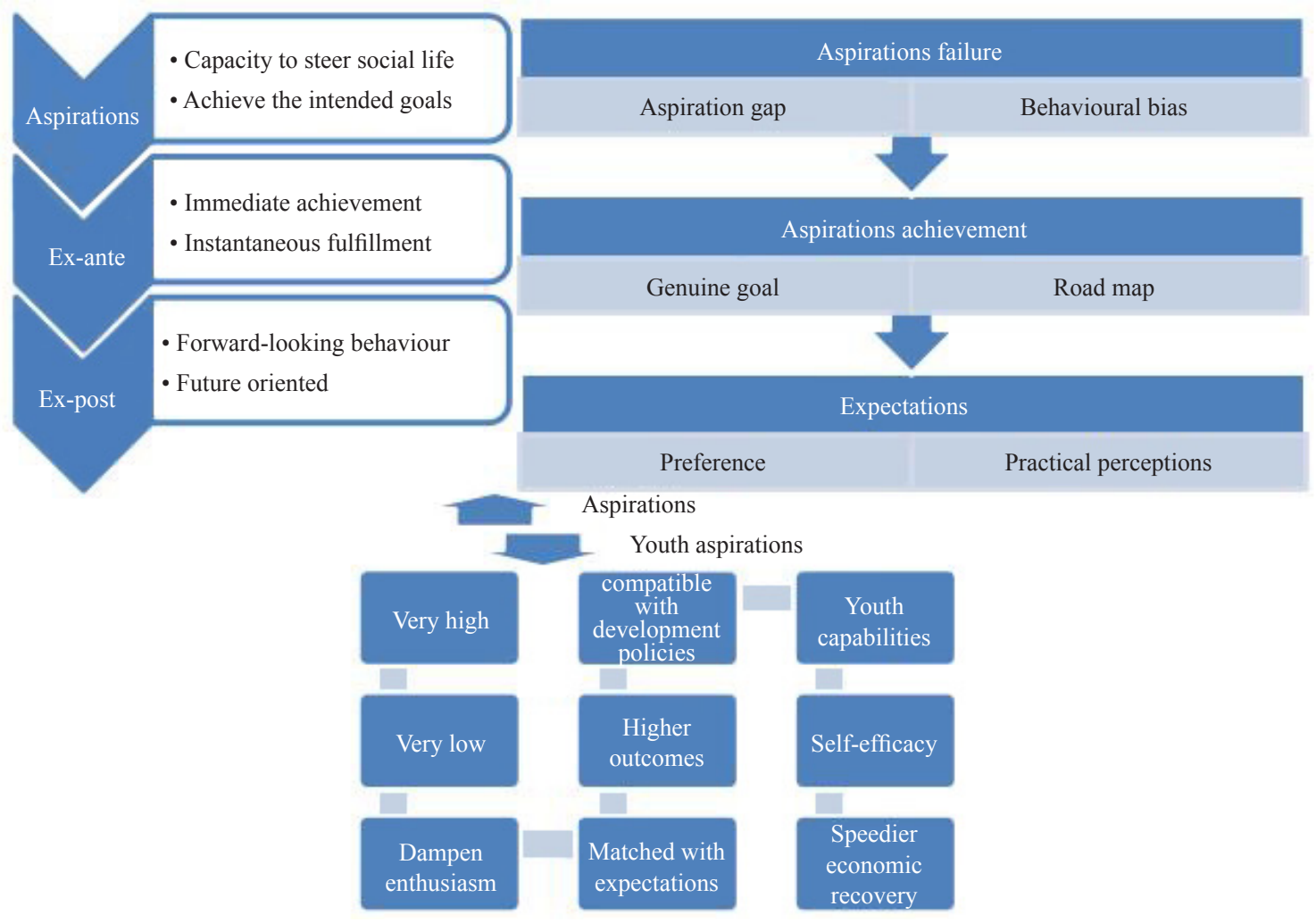

Figure 1. Framework of youth aspirations and economic recovery

\section{Objectives and methodology}

The main objectives of the study are to ascertain the perceptions of the youth regarding economic recovery of Jammu and Kashmir after the abrogation of Article 370, and to draw the policy implications for the speedier economic recovery of conflict-ridden regions. The study has been confined to Jammu region of the Jammu and Kashmir. The study is mainly focused on four selected districts of the Jammu region viz Jammu, Kathua, Rajouri, and Poonch. A cluster sampling technique has been used to select respondents from the selected districts. The study has been restricted to the educated youth of selected districts as they represent the hope for the future and they can better interpret economic recovery of Jammu and Kashmir and also help to understand their perceptions, attitudes and expectations in terms of economic conditions in the coming years. The primary data has been collected through a questionnaire survey of the youth of selected districts. A total of 120 educated youth (graduates, postgraduates, research degree holders belonging to different religious groups viz. Hindus and Muslims: 40 each, and Christians and Sikhs: 20 each) have been selected based on their approximate proportional representation in the total population. Data has been analyzed using simple descriptive statistics. 


\section{Profile of sample area}

The Article 370 of the Constitution of India granted special status to Jammu and Kashmir State, a region situated in the Himalayan Mountains of northern India. In the later half of 2019, the Government of India revoked the special status and divided the Jammu and Kashmir State into two Union Territories (UTs) viz. Jammu and Kashmir, and Ladakh. The UT of Jammu and Kashmir lies between $32^{\circ} 17^{\prime} \mathrm{N}$ to $36^{\circ} 58^{\prime} \mathrm{N}$ latitudes and $73^{\circ} 26^{\prime} \mathrm{E}$ to $8^{\circ} 26^{\prime}$ E longitudes. Jammu and Kashmir share a boundary with Afghanistan in the north-west, Pakistan in the west, with China in Ladakh sector, Tibet in the East and Punjab and Himachal Pradesh in the South. The map of UT Jammu and Kashmir is shown in Figure 2. Each region has diverse climatic and cultural characteristics. The winter capital is Jammu and the summer capital is Srinagar. The geographical area of Jammu and Kashmir is 42,241 km (16,309 sq). The total districts in Jammu and Kashmir are 20.

Jammu region is situated between $74^{\circ}-24^{\prime \prime}$ and $75^{\circ}-18^{\prime \prime}$ E longitude and $32^{\circ}-50^{\prime \prime}$ and $33^{\circ}-30^{\prime \prime} \mathrm{N}$. It is restricted in the north and northeast by the districts of Reasi and Udhampur, Gurdaspur district in Punjab and Sialkot district of Rawalpindi in the south and south-west and district Rajouri and parts of Bimber in the north-west. Jammu, one of the most crowded regions of the UT. It represents $12.16 \%$ of the total population and rank first in terms of population. It includes a region of $2,342 \mathrm{sq}$. $\mathrm{km}$ with population density of 653 persons per sq. $\mathrm{km}$. Jammu city is the main cultural and economic centers of the Jammu region and linked to other parts of the country by road, rail and air transports and hub of small manufacturing and trade.

The district Kathua is situated at $32^{\circ} 17^{\prime}$ to $32^{\circ} 55^{\prime} \mathrm{N}$ latitude and $75^{\circ} 70^{\prime}$ to $76^{\circ} 16^{\prime}$ E longitudes and lies at the southernmost part of Jammu and Kashmir. The district is encompassed by Punjab in the south-east, Doda, and Udhampur in north and north-west, Jammu in the west, Pakistan in the south-west. The district experienced a wide range of the atmosphere from sub-tropical to calm and indeed, even Alpine in higher locales. Agriculture and horticulture are dominant economic activities. Rajouri district is encircled by district Poonch in the north side, Jammu in the south, Reasi in the east, and Mirpur in the west. It covers an area of 26,430 sq. km. and the environment differs from semitropical in the southern part to mild in the mountainous northern part. People are mainly agriculture-oriented.

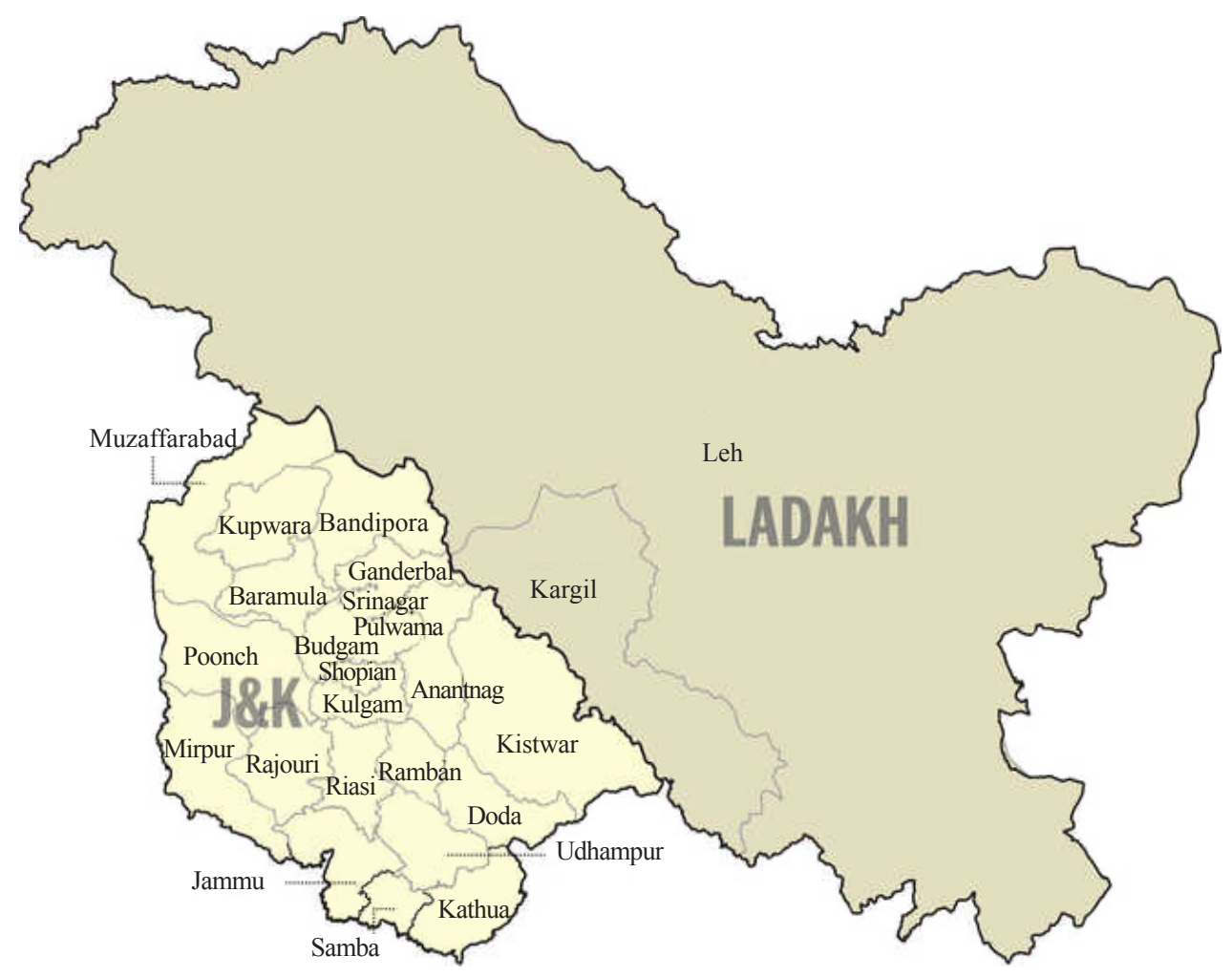

Figure 2. Map of Jammu and Kashmir 
Poonch district with an area of 1674 sq. is located at $33^{\circ} 7^{\prime} \mathrm{N}$ to $74^{\circ} 07^{\prime} \mathrm{E}$ with an altitude of 981 meters. It is bounded by Baramula, Budgam and Pulwama districts from north and northeast and district Rajouri on the east and Pakistan on the west with mountainous and hilly topography. Poonch is a small district but rich in forests with a cooler climate and predominantly rural and agriculture oriented.

\section{Results of the study}

The Article 370 under the Constitution of India was considered as the primary cause of economic backwardness of Jammu and Kashmir due to inadequate private and foreign investment in education, health, horticulture, handicrafts and tourism and also rampant corruption, poverty, unaccountability, high incidence of horizontal inequalities, lack of agriculture reforms, low industrialization, youth violence, unemployment, terrorism, and poor implementation of economic policies. All these factors denied Jammu and Kashmir to utilize the full productive capacity. Keeping the above in view, the Government of India abrogated Article 370 to put the Jammu and Kashmir on the path of economic growth by attracting substantial private investment in education, health, and tourism and creating employment opportunities. The abrogation of Article 370 will positively impact the people to usher in development and modernization of Jammu and Kashmir.

\subsection{Age, religion and educational profile of youth}

Table 1. Age profile of sample youth (No./\%)

\begin{tabular}{cccccc}
\hline \multirow{2}{*}{ Age Profile } & \multicolumn{5}{c}{ Districts } \\
\cline { 2 - 6 } & Jammu & Kathua & Rajouri & Poonch & N \\
\cline { 2 - 6 } $20-25$ & 19 & 11 & 06 & 13 & 49 \\
& $(38.7)^{\mathrm{a}}$ & $(40.7)^{\mathrm{a}}$ & $(30.0)^{\mathrm{a}}$ & $(54.1)^{\mathrm{a}}$ & $(40.8)^{\mathrm{a}}$ \\
& $(38.7)^{\mathrm{b}}$ & $(22.4)^{\mathrm{b}}$ & $(12.2)^{\mathrm{b}}$ & $(26.5)^{\mathrm{b}}$ & $(100.0)^{\mathrm{b}}$ \\
$25-30$ & 28 & 14 & 14 & 09 & 65 \\
& $(57.1)^{\mathrm{a}}$ & $(51.8)^{\mathrm{a}}$ & $(70.0)^{\mathrm{a}}$ & $(37.5)^{\mathrm{a}}$ & $(54.2)^{\mathrm{a}}$ \\
& $(43.1)^{\mathrm{b}}$ & $(21.5)^{\mathrm{b}}$ & $(21.5)^{\mathrm{b}}$ & $(13.8)^{\mathrm{b}}$ & $(100.0)^{\mathrm{b}}$ \\
& 02 & 02 & & 02 & 06 \\
& $(4.1)^{\mathrm{a}}$ & $(7.04)^{\mathrm{a}}$ & - & $(8.03)^{\mathrm{a}}$ & $(5.00)^{\mathrm{a}}$ \\
& $(33.3)^{\mathrm{b}}$ & $(33.3)^{\mathrm{b}}$ & & $(33.3)^{\mathrm{b}}$ & $(100.0)^{\mathrm{b}}$ \\
& 49 & 27 & 20 & 24 & 120 \\
$\mathrm{~N}$ & $(40.8)^{\mathrm{b}}$ & $(22.5)^{\mathrm{b}}$ & $(16.6)^{\mathrm{b}}$ & $(20.0)^{\mathrm{b}}$ & $(100.0)^{\mathrm{b}}$ \\
& $(100.0)^{\mathrm{a}}$ & $(100.0)^{\mathrm{a}}$ & $(100.0)^{\mathrm{a}}$ & $(100.0)^{\mathrm{a}}$ & $(100.0)^{\mathrm{a}}$ \\
\hline
\end{tabular}

Source: Survey Data

Note: Data in parenthesis shows percentage

a: shows column-wise percentage

b: shows row-wise percentage

Table 1 reveals that more than half of the sampled youth were in the age group of 25-30 years, followed by 2025 years $(40.8 \%)$ and $30-35$ years (5\%). Youth aged $25-30$ years constitutes $43.1 \%$ in Jammu, 21.5\% each in Kathua and Rajouri and $13.8 \%$ in Poonch district. Table 2 reveals that Muslims and Hindus youth were in the same proportion (33.3\% each) followed by Sikhs and Christians (16.7\% each). Hindus and Muslims were dominant in district Jammu (50\%), while Sikhs were dominant in Poonch and Christians were concentrated more in districts of Jammu and Kathua compared to other districts of Rajouri and Poonch. Table 3 reveals that $41.7 \%$ of sampled educated youth got their education in arts stream, followed by science (32.5\%), commerce (15.0\%), and law (10.8\%). In Jammu district, 44\% of sampled youth were educated in the arts, while in the districts of Rajouri and Poonch, a significant proportion of 
sampled youth were in the science stream and stood at $17.9 \%$ and $23.1 \%$ respectively, whereas $38.4 \%$ of them were educated in law stream in Kathua district. About $40.8 \%$ of sampled youth were postgraduates followed by M. Phil $(25.8 \%)$, graduates $(16.07 \%)$ and $\mathrm{Ph} . \mathrm{D}(16.7 \%)$. Thus, almost all sampled youth were highly qualified.

Table 2. Religion of sample youth (No./\%)

\begin{tabular}{|c|c|c|c|c|c|}
\hline \multirow{2}{*}{ Religion } & \multicolumn{5}{|c|}{ Districts } \\
\hline & Jammu & Kathua & Rajouri & Poonch & $\mathrm{N}$ \\
\hline \multirow[t]{3}{*}{ Hindu } & 20 & 07 & 06 & 07 & 40 \\
\hline & $(40.8)^{\mathrm{a}}$ & $(25.9)^{\mathrm{a}}$ & $(30.0)^{\mathrm{a}}$ & $(29.2)^{\mathrm{a}}$ & $(33.3)^{\mathrm{a}}$ \\
\hline & $(50.0)^{\mathrm{b}}$ & $(17.5)^{b}$ & $(15.0)^{\mathrm{b}}$ & $(17.5)^{b}$ & $(100.0)^{b}$ \\
\hline \multirow[t]{3}{*}{ Muslim } & 20 & 07 & 10 & 03 & 40 \\
\hline & $(40.8)^{\mathrm{a}}$ & $(25.9)^{\mathrm{a}}$ & $(50.0)^{\mathrm{a}}$ & $(12.5)^{\mathrm{a}}$ & $(33.3)^{\mathrm{a}}$ \\
\hline & $(50.0)^{\mathrm{b}}$ & $(17.5)^{\mathrm{b}}$ & $(25.0)^{\mathrm{b}}$ & $(7.5)^{\mathrm{b}}$ & $(100.0)^{b}$ \\
\hline \multirow[t]{3}{*}{ Sikh } & 03 & 06 & 01 & 10 & 20 \\
\hline & $(6.1)^{\mathrm{a}}$ & $(22.2)^{\mathrm{a}}$ & $(10.0)^{\mathrm{a}}$ & $(41.6)^{\mathrm{a}}$ & $(16.7)^{\mathrm{a}}$ \\
\hline & $(15.0)^{\mathrm{b}}$ & $(30.0)^{b}$ & $(5.0)^{\mathrm{b}}$ & $(50.0)^{\mathrm{b}}$ & $(100.0)^{b}$ \\
\hline \multirow[t]{3}{*}{ Christian } & 06 & 07 & 03 & 04 & 20 \\
\hline & $(12.2)^{\mathrm{a}}$ & $(25.9)^{\mathrm{a}}$ & $(30.0)^{\mathrm{a}}$ & $(16.6)^{\mathrm{a}}$ & $(16.7)^{\mathrm{a}}$ \\
\hline & $(30.0)^{\mathrm{b}}$ & $(35.0)^{\mathrm{b}}$ & $(15.0)^{\mathrm{b}}$ & $(20.0)^{\mathrm{b}}$ & $(100.0)^{b}$ \\
\hline \multirow[t]{3}{*}{$\mathrm{N}$} & 49 & 27 & 20 & 24 & 120 \\
\hline & $(40.8)^{\mathrm{b}}$ & $(22.3)^{\mathrm{b}}$ & $(16.4)^{\mathrm{b}}$ & $(20.0)^{\mathrm{b}}$ & $(100.0)^{\mathrm{b}}$ \\
\hline & $(100.0)^{\mathrm{a}}$ & $(100.0)^{\mathrm{a}}$ & $(100.0)^{\mathrm{a}}$ & $(100.0)^{\mathrm{a}}$ & $(100.0)^{\mathrm{a}}$ \\
\hline
\end{tabular}

Source: Same as Table 1

Note: Same as Table 1

Table 3. Educational streams of sample youth (No./\%)

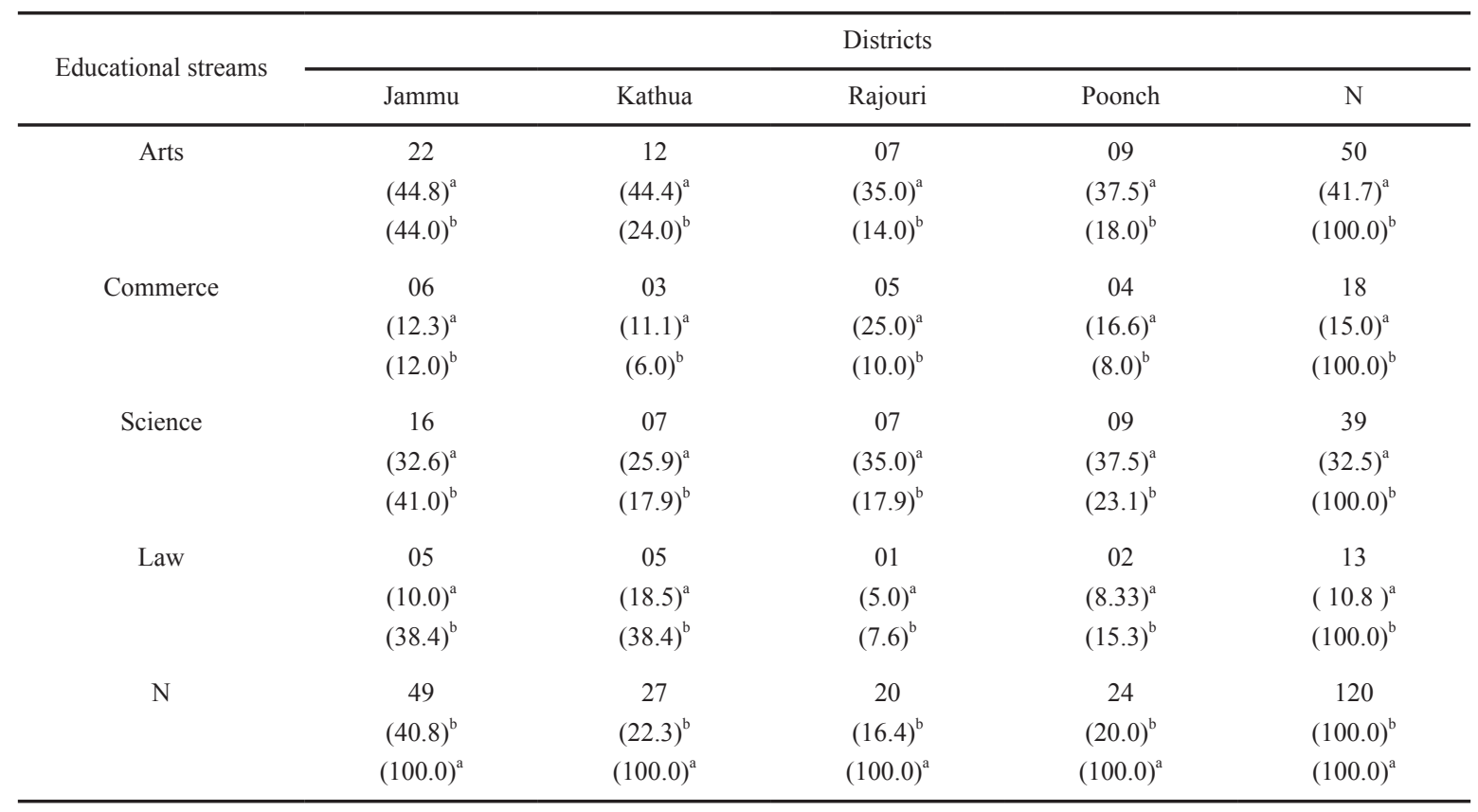

Source: Same as Table 1

Note: Same as Table 1 


\subsection{Knowledge and expectation of State Domestic Product}

Table 4 reveals that the majority of the sampled youth (78.3\%) have knowledge about State Domestic Product (SDP). About $61.7 \%$ of them revealed that agriculture contributes significantly to SDP, followed by the service sector (35.1\%) and the manufacturing sector $(3.1 \%)$. However, there were significant variations in responses in terms of district-wise analysis. For instance, the service sector contributed significantly in the Jammu district (45.4\%), followed by agriculture (37.9\%), and manufacturing (33.3\%). About $60.6 \%$ of sampled youth perceived that the SDP of Jammu and Kashmir stood low compared to other States of India due to lack of productive investment and ill conceived economic policies. With the new political and economic regime with abrogation of Article 370, 29.3\% of sampled youth expected an economic recovery $(26.6 \%)$ and improvement in agriculture $(18.6 \%)$, services $(29.3 \%)$, and manufacturing $(25.3 \%)$ due to enormous private investment opportunities in different sectors. The overall expectation of economic recovery including sectoral recovery has been expressed by $62.5 \%$ of youth (see Table 5 ).

Table 4. Knowledge about State Domestic Product (SDP) (No./\%)

\begin{tabular}{cccccc}
\hline \multirow{2}{*}{ Knowledge } & \multicolumn{5}{c}{ Districts } \\
\cline { 2 - 6 } & Jammu & Kathua & Rajouri & Poonch & $\mathrm{N}$ \\
\hline Yes & 38 & 22 & 15 & 19 & 94 \\
& $(77.5)^{\mathrm{a}}$ & $(81.4)^{\mathrm{a}}$ & $(75.0)^{\mathrm{a}}$ & $(79.7)^{\mathrm{a}}$ & $(78.3)^{\mathrm{a}}$ \\
& $(40.4)^{\mathrm{b}}$ & $(23.4)^{\mathrm{b}}$ & $(15.9)^{\mathrm{b}}$ & $(20.2)^{\mathrm{b}}$ & $(100.0)^{\mathrm{b}}$ \\
No & 11 & 05 & 05 & 05 & 26 \\
& $(22.4)^{\mathrm{a}}$ & $(18.5)^{\mathrm{a}}$ & $(25.0)^{\mathrm{a}}$ & $(20.8)^{\mathrm{a}}$ & $(21.6)^{\mathrm{a}}$ \\
& $(42.3)^{\mathrm{b}}$ & $(19.2)^{\mathrm{b}}$ & $(19.2)^{\mathrm{b}}$ & $(19.2)^{\mathrm{b}}$ & $(100.0)^{\mathrm{b}}$ \\
$\mathrm{N}$ & 49 & 27 & 20 & 24 & 120 \\
& $(40.8)^{\mathrm{a}}$ & $(22.5)^{\mathrm{a}}$ & $(16.6)^{\mathrm{a}}$ & $(20.0)^{\mathrm{a}}$ & $(100.0)^{\mathrm{a}}$ \\
& $(100.0)^{\mathrm{b}}$ & $(100.0)^{\mathrm{b}}$ & $(100.0)^{\mathrm{b}}$ & $(100.0)^{\mathrm{b}}$ & $(100.0)^{\mathrm{b}}$ \\
\hline
\end{tabular}

Source: Same as Table 1

Note: Same as Table 1

Table 5. Expectations of economic recovery and sectoral improvement

\begin{tabular}{|c|c|c|c|c|c|}
\hline $\begin{array}{l}\text { Expectations of } \\
\text { economic recovery }\end{array}$ & \multicolumn{5}{|c|}{ Districts } \\
\hline \multirow[t]{2}{*}{ Economic recovery } & 08 & 05 & 02 & 05 & 20 \\
\hline & $(40.0)^{\mathrm{b}}$ & $(25.0)^{\mathrm{b}}$ & $(10.0)^{\mathrm{b}}$ & $(25.0)^{\mathrm{b}}$ & $(100.0)^{\mathrm{b}}$ \\
\hline $\begin{array}{l}\text { Agriculture } \\
\text { Recovery }\end{array}$ & $(28.5)^{\mathrm{b}}$ & $(14.3)^{b}$ & $(21.4)^{\mathrm{b}}$ & $(35.7)^{\mathrm{b}}$ & $(100.0)^{\mathrm{b}}$ \\
\hline \multirow[t]{3}{*}{ Service recovery } & 08 & 06 & 04 & 04 & 22 \\
\hline & $(25.0)^{\mathrm{a}}$ & $(42.8)^{\mathrm{a}}$ & $(30.7)^{\mathrm{a}}$ & $(25.0)^{\mathrm{a}}$ & $(29.3)^{\mathrm{a}}$ \\
\hline & $(36.4)^{b}$ & $(27.2)^{b}$ & $(18.1)^{b}$ & $(18.1)^{\mathrm{b}}$ & $(100.0)^{b}$ \\
\hline \multirow[t]{3}{*}{$\mathrm{N}$} & 32 & 14 & 13 & 16 & 75 \\
\hline & $(42.6)^{\mathrm{b}}$ & $(18.6)^{\mathrm{b}}$ & $(17.3)^{\mathrm{b}}$ & $(21.3)^{\mathrm{b}}$ & $(100.0)^{\mathrm{b}}$ \\
\hline & $(100.0)^{\mathrm{a}}$ & $(100.0)^{\mathrm{a}}$ & $(100.0)^{\mathrm{a}}$ & $(100.0)^{\mathrm{a}}$ & $(100.0)^{\mathrm{a}}$ \\
\hline
\end{tabular}

Source: Same as Table 1

Note: Same as Table 1 


\subsection{Expectation of unemployment}

Table 6. Expectation of unemployment by sample youth (No./\%)

\begin{tabular}{cccccc}
\hline \multirow{2}{*}{ Expectation } & \multicolumn{5}{c}{ Districts } \\
\cline { 2 - 6 } & Jammu & Kathua & Rajouri & Poonch & $\mathrm{N}$ \\
\hline Very high & 30 & 11 & 08 & 12 & 61 \\
& $(61.2)^{\mathrm{a}}$ & $(40.7)^{\mathrm{a}}$ & $(40.0)^{\mathrm{a}}$ & $(50.0)^{\mathrm{a}}$ & $(50.8)^{\mathrm{a}}$ \\
& $(49.1)^{\mathrm{b}}$ & $(18.0)^{\mathrm{b}}$ & $(13.1)^{\mathrm{b}}$ & $(19.6)^{\mathrm{b}}$ & $(100.0)^{\mathrm{b}}$ \\
High & 13 & 11 & 09 & 07 & 40 \\
& $(26.5)^{\mathrm{a}}$ & $(40.7)^{\mathrm{a}}$ & $(45.0)^{\mathrm{a}}$ & $(29.1)^{\mathrm{a}}$ & $(33.3)^{\mathrm{a}}$ \\
& $(32.5)^{\mathrm{b}}$ & $(27.5)^{\mathrm{b}}$ & $(22.5)^{\mathrm{b}}$ & $(17.5)^{\mathrm{b}}$ & $(100.0)^{\mathrm{b}}$ \\
Low & 04 & 03 & 01 & 03 & 11 \\
& $(8.1)^{\mathrm{a}}$ & $(11.1)^{\mathrm{a}}$ & $(5.0)^{\mathrm{a}}$ & $(12.5)^{\mathrm{a}}$ & $(9.2)^{\mathrm{a}}$ \\
Very low & $(36.4)^{\mathrm{b}}$ & $(27.3)^{\mathrm{b}}$ & $(9.1)^{\mathrm{b}}$ & $(27.3)^{\mathrm{b}}$ & $(100.0)^{\mathrm{b}}$ \\
& 02 & 02 & 02 & 01 & 08 \\
& $(4.2)^{\mathrm{a}}$ & $(7.4)^{\mathrm{a}}$ & $(10.0)^{\mathrm{a}}$ & $(4.1)^{\mathrm{a}}$ & $(6.7)^{\mathrm{a}}$ \\
$\mathrm{N}$ & $(25.0)^{\mathrm{b}}$ & $(25.0)^{\mathrm{b}}$ & $(25.0)^{\mathrm{b}}$ & $(12.5)^{\mathrm{b}}$ & $(100.0)^{\mathrm{b}}$ \\
& 49 & 27 & 20 & 24 & 120 \\
& $(40.8)^{\mathrm{b}}$ & $(22.5)^{\mathrm{b}}$ & $(16.6)^{\mathrm{b}}$ & $(20.0)^{\mathrm{b}}$ & $(100.0)^{\mathrm{a}}$ \\
& $(100.0)^{\mathrm{a}}$ & $(100.0)^{\mathrm{a}}$ & $(100.0)^{\mathrm{a}}$ & $(100.0)^{\mathrm{a}}$ & $(100.0)^{\mathrm{b}}$ \\
\hline
\end{tabular}

Source: Same as Table 1

Note: Same as Table 1

Table 7. Barriers of educated unemployment (No./\%)

\begin{tabular}{|c|c|c|c|c|c|}
\hline \multirow{2}{*}{ Barriers } & \multicolumn{5}{|c|}{ Districts } \\
\hline & Jammu & Kathua & Rajouri & Poonch & $\mathrm{N}$ \\
\hline $\begin{array}{l}\text { Inadequate access } \\
\text { to information }\end{array}$ & $\begin{array}{c}06 \\
(5.7)^{\mathrm{a}} \\
(28.5)^{\mathrm{b}}\end{array}$ & $\begin{array}{c}07 \\
(10.6)^{\mathrm{a}} \\
(33.3)^{\mathrm{b}}\end{array}$ & $\begin{array}{c}04 \\
(11.4)^{\mathrm{a}} \\
(19.1)^{\mathrm{b}}\end{array}$ & $\begin{array}{c}04 \\
(7.1)^{\mathrm{a}} \\
(19.1)^{\mathrm{b}}\end{array}$ & $\begin{array}{c}21 \\
(8.04)^{\mathrm{a}} \\
(100.0)^{\mathrm{b}}\end{array}$ \\
\hline $\begin{array}{l}\text { Not enough } \\
\text { government jobs }\end{array}$ & $\begin{array}{c}14 \\
(13.4)^{\mathrm{a}} \\
(53.8)^{\mathrm{b}}\end{array}$ & $\begin{array}{c}09 \\
(13.6)^{\mathrm{a}} \\
(34.6)^{\mathrm{b}}\end{array}$ & $\begin{array}{c}01 \\
(2.8)^{\mathrm{a}} \\
(3.8)^{\mathrm{b}}\end{array}$ & $\begin{array}{c}02 \\
(3.5)^{\mathrm{a}} \\
(7.6)^{\mathrm{b}}\end{array}$ & $\begin{array}{c}26 \\
(9.96)^{\mathrm{a}} \\
(100.0)^{\mathrm{b}}\end{array}$ \\
\hline Lack of soft skills & $\begin{array}{c}07 \\
(6.7)^{\mathrm{a}} \\
(38.8)^{\mathrm{b}}\end{array}$ & $\begin{array}{c}04 \\
(6.1)^{\mathrm{a}} \\
(22.2)^{\mathrm{b}}\end{array}$ & $\begin{array}{c}03 \\
(8.6)^{\mathrm{a}} \\
(16.6)^{\mathrm{b}}\end{array}$ & $\begin{array}{c}04 \\
(7.1)^{\mathrm{a}} \\
(22.2)^{\mathrm{b}}\end{array}$ & $\begin{array}{c}18 \\
(6.8)^{\mathrm{a}} \\
(100.0)^{\mathrm{b}}\end{array}$ \\
\hline $\begin{array}{l}\text { Less aware of } \\
\text { job openings }\end{array}$ & $\begin{array}{c}36 \\
(34.6)^{\mathrm{a}} \\
(42.3)^{\mathrm{b}}\end{array}$ & $\begin{array}{c}19 \\
(28.7)^{\mathrm{a}} \\
(22.4)^{\mathrm{b}}\end{array}$ & $\begin{array}{c}10 \\
(28.5)^{\mathrm{a}} \\
(11.7)^{\mathrm{b}}\end{array}$ & $\begin{array}{c}20 \\
(35.7)^{\mathrm{a}} \\
(23.5)^{\mathrm{b}}\end{array}$ & $\begin{array}{c}85 \\
(32.5)^{\mathrm{a}} \\
(100.0)^{\mathrm{b}}\end{array}$ \\
\hline Lack of good jobs & $\begin{array}{c}17 \\
(16.3)^{\mathrm{a}} \\
(20.0)^{\mathrm{b}}\end{array}$ & $\begin{array}{c}11 \\
(16.6)^{\mathrm{a}} \\
(12.9)^{\mathrm{b}}\end{array}$ & $\begin{array}{c}07 \\
(20.0)^{\mathrm{a}} \\
(8.2)^{\mathrm{b}}\end{array}$ & $\begin{array}{c}09 \\
(16.1)^{\mathrm{a}} \\
(10.5)^{\mathrm{b}}\end{array}$ & $\begin{array}{c}44 \\
(16.8)^{\mathrm{a}} \\
(100.0)^{\mathrm{b}}\end{array}$ \\
\hline Lack of education & $\begin{array}{c}10 \\
(9.6)^{\mathrm{a}} \\
(33.3)^{\mathrm{b}}\end{array}$ & $\begin{array}{c}05 \\
(7.5)^{\mathrm{a}} \\
(16.6)^{\mathrm{b}}\end{array}$ & $\begin{array}{c}06 \\
(17.1)^{\mathrm{a}} \\
(20.0)^{\mathrm{b}}\end{array}$ & $\begin{array}{c}09 \\
(16.1)^{\mathrm{a}} \\
(30.0)^{\mathrm{b}}\end{array}$ & $\begin{array}{c}30 \\
(11.4)^{\mathrm{a}} \\
(100.0)^{\mathrm{b}}\end{array}$ \\
\hline $\begin{array}{c}\text { Lack of } \\
\text { good guidance }\end{array}$ & $\begin{array}{c}10 \\
(9.6)^{\mathrm{a}} \\
(47.6)^{\mathrm{b}}\end{array}$ & $\begin{array}{c}06 \\
(9.09)^{\mathrm{a}} \\
(28.5)^{\mathrm{b}}\end{array}$ & $\begin{array}{c}03 \\
(8.5)^{\mathrm{a}} \\
(14.2)^{\mathrm{b}}\end{array}$ & $\begin{array}{c}02 \\
(3.5)^{\mathrm{a}} \\
(9.5)^{\mathrm{b}}\end{array}$ & $\begin{array}{c}21 \\
(8.04)^{\mathrm{a}} \\
(100.0)^{\mathrm{b}}\end{array}$ \\
\hline $\begin{array}{c}\text { Lack of } \\
\text { work experience }\end{array}$ & $\begin{array}{c}04 \\
(3.8)^{\mathrm{a}} \\
(25.0)^{\mathrm{b}}\end{array}$ & $\begin{array}{c}05 \\
(7.5)^{\mathrm{a}} \\
(31.3)^{\mathrm{b}}\end{array}$ & $\begin{array}{c}01 \\
(2.8)^{\mathrm{a}} \\
(6.3)^{\mathrm{b}}\end{array}$ & $\begin{array}{c}06 \\
(10.7)^{\mathrm{a}} \\
(37.5)^{\mathrm{b}}\end{array}$ & $\begin{array}{c}16 \\
(6.1)^{\mathrm{a}} \\
(100.0)\end{array}$ \\
\hline $\mathrm{N}$ & $\begin{array}{c}104 \\
(39.8)^{\mathrm{b}} \\
(100.0)^{\mathrm{a}}\end{array}$ & $\begin{array}{c}66 \\
(25.3)^{\mathrm{b}} \\
(100.0)^{\mathrm{a}}\end{array}$ & $\begin{array}{c}35 \\
(13.4)^{\mathrm{b}} \\
(100.0)^{\mathrm{a}}\end{array}$ & $\begin{array}{c}56 \\
(21.4)^{\mathrm{b}} \\
(100.0)^{\mathrm{a}}\end{array}$ & $\begin{array}{c}261 \\
(100.0)^{\mathrm{b}} \\
(100.0)^{\mathrm{a}}\end{array}$ \\
\hline
\end{tabular}

Source: Same as Table 1

Note: Same as Table 1 
Table 6 reveals that more than half of sampled youth perceived that the unemployment rate was very high (50.8\%) and high (33.3\%), followed by low (12.5\%) and very low (4.1\%) due to slow economic recovery. Unemployment was high in district Jammu compared to other districts of the Jammu region. The main barriers of educated unemployment include lack of education, job openings, soft skills, public sector jobs, and work experience with significant variation across the selected districts (see Table 7). These barriers persisted due to closed economy, reliance on government jobs, unorganized and unskilled workers in tourism sector, and use of traditional methods in agriculture including inadequate awareness of self-employment schemes. Table 8 reveals the expectations of sampled youth on prospective job opportunities in the next 5 years. Only $10.8 \%$ of them opined that employment is expected to increase due to investment in different sectors including tourism, while an equal proportion of them also reported that unemployment is likely to rapidly increase after abrogation of Article 370 due to indifferent approach towards the youth.

Table 8. Expectations of job opportunities in the next 5 years (No./\%)

\begin{tabular}{|c|c|c|c|c|c|}
\hline \multirow{2}{*}{ Expectations } & \multicolumn{5}{|c|}{ Districts } \\
\hline & Jammu & Kathua & Rajouri & Poonch & $\mathrm{N}$ \\
\hline Increase & $\begin{array}{c}16 \\
(32.6)^{\mathrm{a}} \\
(35.5)^{\mathrm{b}}\end{array}$ & $\begin{array}{c}13 \\
(48.1)^{\mathrm{a}} \\
(28.8)^{\mathrm{b}}\end{array}$ & $\begin{array}{c}04 \\
(20.0)^{\mathrm{a}} \\
(8.8)^{\mathrm{b}}\end{array}$ & $\begin{array}{c}12 \\
(50.0)^{\mathrm{a}} \\
(26.6)^{\mathrm{b}}\end{array}$ & $\begin{array}{c}45 \\
(37.5)^{\mathrm{a}} \\
(100.0)^{\mathrm{b}}\end{array}$ \\
\hline Decrease & $\begin{array}{c}03 \\
(6.1)^{\mathrm{a}} \\
(23.1)^{\mathrm{b}}\end{array}$ & $\begin{array}{c}04 \\
(14.8)^{\mathrm{a}} \\
(30.7)^{\mathrm{b}}\end{array}$ & $\begin{array}{c}04 \\
(20.0)^{\mathrm{a}} \\
(30.7)^{\mathrm{b}}\end{array}$ & $\begin{array}{c}02 \\
(8.3)^{\mathrm{a}} \\
(15.4)^{\mathrm{b}}\end{array}$ & $\begin{array}{c}13 \\
(10.8)^{\mathrm{a}} \\
(100.0)^{\mathrm{b}}\end{array}$ \\
\hline Rapidly increase & $\begin{array}{c}01 \\
(2.04)^{\mathrm{a}} \\
(16.6)^{\mathrm{b}}\end{array}$ & $\begin{array}{c}02 \\
(7.40)^{\mathrm{a}} \\
(33.3)^{\mathrm{b}}\end{array}$ & $\begin{array}{c}01 \\
(5.0)^{\mathrm{a}} \\
(16.6)^{\mathrm{b}}\end{array}$ & $\begin{array}{c}02 \\
(8.3)^{\mathrm{a}} \\
(33.3)^{\mathrm{b}}\end{array}$ & $\begin{array}{c}06 \\
(5.0)^{\mathrm{a}} \\
(100.0)^{\mathrm{b}}\end{array}$ \\
\hline Rapidly decrease & $\begin{array}{c}08 \\
(16.3)^{\mathrm{a}} \\
(47.1)^{\mathrm{b}}\end{array}$ & $\begin{array}{c}03 \\
(11.1)^{\mathrm{a}} \\
(17.6)^{\mathrm{b}}\end{array}$ & $\begin{array}{c}04 \\
(20.0)^{\mathrm{a}} \\
(23.5)^{\mathrm{b}}\end{array}$ & $\begin{array}{c}02 \\
(8.3)^{\mathrm{a}} \\
(11.7)^{\mathrm{b}}\end{array}$ & $\begin{array}{c}17 \\
(14.2)^{\mathrm{a}} \\
(100.0)^{\mathrm{b}}\end{array}$ \\
\hline Cannot say & $\begin{array}{c}21 \\
(42.8)^{\mathrm{a}} \\
(53.8)^{\mathrm{b}}\end{array}$ & $\begin{array}{c}05 \\
(18.5)^{\mathrm{a}} \\
(12.8)^{\mathrm{b}}\end{array}$ & $\begin{array}{c}07 \\
(35.0)^{\mathrm{a}} \\
(17.9)^{\mathrm{b}}\end{array}$ & $\begin{array}{c}06 \\
(25.0)^{\mathrm{a}} \\
(15.4)^{\mathrm{b}}\end{array}$ & $\begin{array}{c}39 \\
(32.5)^{\mathrm{a}} \\
(100.0)^{\mathrm{b}}\end{array}$ \\
\hline $\mathrm{N}$ & $\begin{array}{c}49 \\
(40.8)^{\mathrm{b}} \\
(100.0)^{\mathrm{a}}\end{array}$ & $\begin{array}{c}27 \\
(22.5)^{\mathrm{b}} \\
(100.0)^{\mathrm{a}}\end{array}$ & $\begin{array}{c}20 \\
(16.6)^{\mathrm{b}} \\
(100.0)^{\mathrm{a}}\end{array}$ & $\begin{array}{c}24 \\
(20.0)^{\mathrm{b}} \\
(100.0)^{\mathrm{a}}\end{array}$ & $\begin{array}{c}120 \\
(100.0)^{\mathrm{b}} \\
(100.0)^{\mathrm{a}}\end{array}$ \\
\hline
\end{tabular}

Source: Same as Table 1

Note: Same as Table 1

\subsection{Educational and health expectations of youth}

The majority of sampled youth have attained high educational qualifications. Table 9 reveals that nearly onefourth were not satisfied with educational achievement before abrogation of the Article 370. Sampled youth expected improvement in educational facilities in the near future through more emphasis on practical skills, quality of libraries, modernize educational programs, use of modern teaching methods, market-oriented courses, better teachers and better educational environment (see Table 10). Table 11 reveals that more than two-thirds of sampled youth have reported inadequate availability of health facilities due to high cost, lack of specialist doctors, inaccessibility, inadequate awareness, lack of health infrastructure besides poor implementation of financial and social security and health measures. Table 12 reveals that nearly half of them expected an improvement in health facilities in near future and $8.3 \%$ of them expected no improvement in health facilities under new regime due to callous attitude of health administration and policymakers. The marginalized population remained discriminated compared to mainstream population, which required an end to discrimination of marginalized people, seek out marginalized choices, inclusion and wider support to 
marginalized movements. About one-fourth of sampled youth expected that discrimination of marginalized sections can be reduced by supporting marginalized movements.

Table 9. Satisfaction regarding educational qualifications (No./\%)

\begin{tabular}{|c|c|c|c|c|c|}
\hline \multirow{2}{*}{ Expectations } & \multicolumn{5}{|c|}{ Districts } \\
\hline & Jammu & Kathua & Rajouri & Poonch & $\mathrm{N}$ \\
\hline \multirow[t]{3}{*}{ Yes } & 38 & 24 & 10 & 19 & 91 \\
\hline & $(77.5)^{\mathrm{a}}$ & $(88.8)^{\mathrm{a}}$ & $(50.0)^{\mathrm{a}}$ & $(79.7)^{\mathrm{a}}$ & $(75.8)^{\mathrm{a}}$ \\
\hline & $(41.7)^{b}$ & $(26.4)^{\mathrm{b}}$ & $(10.9)^{\mathrm{b}}$ & $(20.8)^{b}$ & $(100.0)^{b}$ \\
\hline \multirow[t]{3}{*}{ No } & 11 & 03 & 10 & 05 & 29 \\
\hline & $(22.5)^{\mathrm{a}}$ & $(11.2)^{\mathrm{a}}$ & $(50.0)^{\mathrm{a}}$ & $(20.8)^{\mathrm{a}}$ & $(24.2)^{\mathrm{a}}$ \\
\hline & $(37.9)^{\mathrm{b}}$ & $(10.3)^{b}$ & $(34.4)^{b}$ & $(17.2)^{b}$ & $(100.0)^{b}$ \\
\hline \multirow[t]{3}{*}{$\mathrm{N}$} & 49 & 02 & 20 & 24 & 120 \\
\hline & $(40.8)^{\mathrm{b}}$ & $(1.6)^{\mathrm{b}}$ & $(16.6)^{b}$ & $(20.0)^{\mathrm{b}}$ & $(100.0)^{b}$ \\
\hline & $(100.0)^{\mathrm{a}}$ & $(100.0)^{\mathrm{a}}$ & $(100.0)^{\mathrm{a}}$ & $(100.0)^{\mathrm{a}}$ & $(100.0)^{\mathrm{a}}$ \\
\hline
\end{tabular}

Source: Same as Table 1

Note: Same as Table 1

Table 10. Necessary things to improve educational institutions (No./\%)

\begin{tabular}{|c|c|c|c|c|c|}
\hline \multirow{2}{*}{$\begin{array}{l}\text { Improving educational } \\
\text { institutions }\end{array}$} & \multicolumn{5}{|c|}{ Districts } \\
\hline & Jammu & Kathua & Rajouri & Poonch & $\mathrm{N}$ \\
\hline $\begin{array}{l}\text { Focus more } \\
\text { on practical skill }\end{array}$ & $\begin{array}{c}32 \\
(18.2)^{\mathrm{a}} \\
(43.2)^{\mathrm{b}}\end{array}$ & $\begin{array}{c}12 \\
(11.6)^{\mathrm{a}} \\
(16.2)^{\mathrm{b}}\end{array}$ & $\begin{array}{c}11 \\
(13.5)^{\mathrm{a}} \\
(14.8)^{\mathrm{b}}\end{array}$ & $\begin{array}{c}19 \\
(20.4)^{\mathrm{a}} \\
(25.6)^{\mathrm{b}}\end{array}$ & $\begin{array}{c}74 \\
(16.3)^{\mathrm{a}} \\
(100.0)^{\mathrm{b}}\end{array}$ \\
\hline Improve quality of libraries & $\begin{array}{c}15 \\
(8.5)^{\mathrm{a}} \\
(37.5)^{\mathrm{b}}\end{array}$ & $\begin{array}{c}08 \\
(7.7)^{\mathrm{a}} \\
(20.0)^{\mathrm{b}}\end{array}$ & $\begin{array}{c}10 \\
(12.3)^{\mathrm{a}} \\
(25.0)^{\mathrm{b}}\end{array}$ & $\begin{array}{c}07 \\
(7.5)^{\mathrm{a}} \\
(17.5)^{\mathrm{b}}\end{array}$ & $\begin{array}{c}40 \\
(8.8)^{\mathrm{a}} \\
(100.0)^{\mathrm{b}}\end{array}$ \\
\hline $\begin{array}{l}\text { Modernize educational } \\
\text { programmes }\end{array}$ & $\begin{array}{c}27 \\
(15.3)^{\mathrm{a}} \\
(41.5)^{\mathrm{b}}\end{array}$ & $\begin{array}{c}14 \\
(13.5)^{\mathrm{a}} \\
(21.5)^{\mathrm{b}}\end{array}$ & $\begin{array}{c}11 \\
(13.5)^{\mathrm{a}} \\
(16.9)^{\mathrm{b}}\end{array}$ & $\begin{array}{c}13 \\
(13.9)^{\mathrm{a}} \\
(20.0)^{\mathrm{b}}\end{array}$ & $\begin{array}{c}65 \\
(14.3)^{\mathrm{a}} \\
(100.0)^{\mathrm{b}}\end{array}$ \\
\hline $\begin{array}{l}\text { Use of modern } \\
\text { teaching methods }\end{array}$ & $\begin{array}{c}21 \\
(11.9)^{\mathrm{a}} \\
(31.3)^{\mathrm{b}}\end{array}$ & $\begin{array}{c}19 \\
(18.4)^{\mathrm{a}} \\
(28.3)^{\mathrm{b}}\end{array}$ & $\begin{array}{c}11 \\
(13.5)^{\mathrm{a}} \\
(16.4)^{\mathrm{b}}\end{array}$ & $\begin{array}{c}16 \\
(17.2)^{\mathrm{a}} \\
(23.8)^{\mathrm{b}}\end{array}$ & $\begin{array}{c}67 \\
(14.7)^{\mathrm{a}} \\
(100.0)^{\mathrm{b}}\end{array}$ \\
\hline $\begin{array}{l}\text { Modernize content } \\
\text { of courses }\end{array}$ & $\begin{array}{c}31 \\
(17.7)^{\mathrm{a}} \\
(46.3)^{\mathrm{b}}\end{array}$ & $\begin{array}{c}19 \\
(18.4)^{\mathrm{a}} \\
(28.3)^{\mathrm{b}}\end{array}$ & $\begin{array}{c}12 \\
(14.8)^{\mathrm{a}} \\
(17.9)^{\mathrm{b}}\end{array}$ & $\begin{array}{c}11 \\
(11.8)^{\mathrm{a}} \\
(16.4)^{\mathrm{b}}\end{array}$ & $\begin{array}{c}73 \\
(16.1)^{\mathrm{a}} \\
(100.0)^{\mathrm{b}}\end{array}$ \\
\hline Better teachers & $\begin{array}{c}25 \\
(14.2)^{\mathrm{a}} \\
(34.2)^{\mathrm{b}}\end{array}$ & $\begin{array}{c}17 \\
(16.5)^{\mathrm{a}} \\
(23.3)^{\mathrm{b}}\end{array}$ & $\begin{array}{c}15 \\
(18.5)^{\mathrm{a}} \\
(20.5)^{\mathrm{b}}\end{array}$ & $\begin{array}{c}16 \\
(17.2)^{\mathrm{a}} \\
(21.9)^{\mathrm{b}}\end{array}$ & $\begin{array}{c}73 \\
(16.1)^{\mathrm{a}} \\
(100.0)^{\mathrm{b}}\end{array}$ \\
\hline Better environment & $\begin{array}{c}25 \\
(14.2)^{\mathrm{a}} \\
(40.9)^{\mathrm{b}}\end{array}$ & $\begin{array}{c}14 \\
(13.5)^{\mathrm{a}} \\
(22.9)^{\mathrm{b}}\end{array}$ & $\begin{array}{c}11 \\
(13.5)^{\mathrm{a}} \\
(18.0)^{\mathrm{b}}\end{array}$ & $\begin{array}{c}11 \\
(11.8)^{\mathrm{a}} \\
(18.0)^{\mathrm{b}}\end{array}$ & $\begin{array}{c}61 \\
(13.4)^{\mathrm{a}} \\
(100.0)^{\mathrm{b}}\end{array}$ \\
\hline $\mathrm{N}$ & $\begin{array}{c}176 \\
(38.8)^{\mathrm{b}} \\
(100.0)^{\mathrm{a}}\end{array}$ & $\begin{array}{c}103 \\
(22.7)^{\mathrm{b}} \\
(100.0)^{\mathrm{a}}\end{array}$ & $\begin{array}{c}81 \\
(17.8)^{\mathrm{b}} \\
(100.0)^{\mathrm{a}}\end{array}$ & $\begin{array}{c}93 \\
(20.5)^{\mathrm{b}} \\
(100.0)^{\mathrm{a}}\end{array}$ & $\begin{array}{c}453 \\
(100.0)^{\mathrm{b}} \\
(100.0)^{\mathrm{a}}\end{array}$ \\
\hline
\end{tabular}

Source: Same as Table 1

Note: Same as Table 1 
Table 11. Problems with health facilities (No./\%)

\begin{tabular}{|c|c|c|c|c|c|}
\hline \multirow{2}{*}{ Problems } & \multicolumn{5}{|c|}{ Districts } \\
\hline & Jammu & Kathua & Rajouri & Poonch & $\mathrm{N}$ \\
\hline \multirow[t]{3}{*}{ Expensive } & 12 & 05 & 07 & 04 & 28 \\
\hline & $(10.2)^{\mathrm{a}}$ & $(9.6)^{\mathrm{a}}$ & $(14.6)^{\mathrm{a}}$ & $(10.2)^{\mathrm{a}}$ & $(10.9)^{\mathrm{a}}$ \\
\hline & $(42.8)^{\mathrm{b}}$ & $(17.8)^{\mathrm{b}}$ & $(25.0)^{\mathrm{b}}$ & $(14.2)^{\mathrm{b}}$ & $(100.0)^{b}$ \\
\hline \multirow[t]{3}{*}{ Lack of specialist doctors } & 18 & 08 & 08 & 10 & 44 \\
\hline & $(15.4)^{\mathrm{a}}$ & $(15.4)^{\mathrm{a}}$ & $(16.7)^{\mathrm{a}}$ & $(25.7)^{\mathrm{a}}$ & $(17.1)^{\mathrm{a}}$ \\
\hline & $(40.9)^{\mathrm{b}}$ & $(18.2)^{\mathrm{b}}$ & $(18.2)^{b}$ & $(22.7)^{b}$ & $(100.0)^{\mathrm{b}}$ \\
\hline \multirow[t]{3}{*}{ Distance problems } & 20 & 06 & 07 & 06 & 39 \\
\hline & $(17.1)^{\mathrm{a}}$ & $(11.5)^{\mathrm{a}}$ & $(14.6)^{\mathrm{a}}$ & $(15.4)^{\mathrm{a}}$ & $(15.2)^{\mathrm{a}}$ \\
\hline & $(51.3)^{\mathrm{b}}$ & $(15.4)^{\mathrm{b}}$ & $(17.9)^{\mathrm{b}}$ & $(15.4)^{\mathrm{b}}$ & $(100.0)^{b}$ \\
\hline \multirow[t]{3}{*}{ Cost of medical service store } & 10 & 05 & 03 & 02 & 20 \\
\hline & $(8.5)^{\mathrm{a}}$ & $(9.6)^{\mathrm{a}}$ & $(6.25)^{\mathrm{a}}$ & $(5.1)^{\mathrm{a}}$ & $(7.8)^{\mathrm{a}}$ \\
\hline & $(50.0)^{\mathrm{b}}$ & $(25.0)^{\mathrm{b}}$ & $(15.0)$ & $(10.0)^{\mathrm{b}}$ & $(100.0)^{\mathrm{b}}$ \\
\hline \multirow[t]{3}{*}{ Awareness problems } & 15 & 09 & 05 & 04 & 33 \\
\hline & $(12.8)^{\mathrm{a}}$ & $(17.3)^{\mathrm{a}}$ & $(10.4)^{\mathrm{a}}$ & $(10.2)^{\mathrm{a}}$ & $(12.8)^{\mathrm{a}}$ \\
\hline & $(45.4)^{\mathrm{b}}$ & $(27.3)^{\mathrm{b}}$ & $(15.2)^{b}$ & $(12.1)^{\mathrm{b}}$ & $(100.0)^{b}$ \\
\hline \multirow[t]{3}{*}{ Formalities for registration } & 14 & 08 & 04 & 06 & 32 \\
\hline & $(11.9)^{\mathrm{a}}$ & $(15.4)^{\mathrm{a}}$ & $(8.3)^{\mathrm{a}}$ & $(15.4)^{\mathrm{a}}$ & $(12.5)^{\mathrm{a}}$ \\
\hline & $(43.7)^{\mathrm{b}}$ & $(25.0)^{\mathrm{b}}$ & $(12.5)^{b}$ & $(18.7)^{\mathrm{b}}$ & $(100.0)^{\mathrm{b}}$ \\
\hline \multirow[t]{3}{*}{ Lack of infrastructure } & 17 & 05 & 08 & 02 & 32 \\
\hline & $(14.5)^{\mathrm{a}}$ & $(9.6)^{\mathrm{a}}$ & $(16.6)^{\mathrm{a}}$ & $(5.1)^{\mathrm{a}}$ & $(12.5)^{\mathrm{a}}$ \\
\hline & $(53.1)^{\mathrm{b}}$ & $(15.6)^{\mathrm{b}}$ & $(25.0)^{\mathrm{b}}$ & $(6.25)^{\mathrm{b}}$ & $(100.0)^{\mathrm{b}}$ \\
\hline \multirow[t]{3}{*}{ High expenses } & 11 & 06 & 06 & 05 & 28 \\
\hline & $(9.6)^{\mathrm{a}}$ & $(11.5)^{\mathrm{a}}$ & $(12.5)^{\mathrm{a}}$ & $(12.8)^{\mathrm{a}}$ & $(10.9)^{\mathrm{a}}$ \\
\hline & $(34.4)^{\mathrm{b}}$ & $(18.7)^{\mathrm{b}}$ & $(18.7)^{\mathrm{b}}$ & $(15.6)^{\mathrm{b}}$ & $(100.0)^{b}$ \\
\hline \multirow[t]{3}{*}{$\mathrm{N}$} & 117 & 52 & 48 & 39 & 256 \\
\hline & $(45.7)^{\mathrm{b}}$ & $(20.3)^{\mathrm{b}}$ & $(18.7)^{b}$ & $(15.2)^{\mathrm{b}}$ & $(100.0)^{b}$ \\
\hline & $(100.0)^{\mathrm{a}}$ & $(100.0)^{\mathrm{a}}$ & $(100.0)^{\mathrm{a}}$ & $(100.0)^{\mathrm{a}}$ & $(100.0)^{\mathrm{a}}$ \\
\hline
\end{tabular}

Source: Same as Table 1

Note: Same as Table 1

Table 12. Expectations regarding improvement in health facilities (No./\%)

\begin{tabular}{|c|c|c|c|c|c|}
\hline \multirow{2}{*}{ Response } & \multicolumn{5}{|c|}{ Districts } \\
\hline & Jammu & Kathua & Rajouri & Poonch & $\mathrm{N}$ \\
\hline \multirow[t]{3}{*}{ Yes } & 20 & 08 & 16 & 15 & 59 \\
\hline & $(40.8)^{\mathrm{a}}$ & $(29.6)^{\mathrm{a}}$ & $(80.0)^{\mathrm{a}}$ & $(62.5)^{\mathrm{a}}$ & $(49.2)^{\mathrm{a}}$ \\
\hline & $(33.8)^{\mathrm{b}}$ & $(13.5)^{\mathrm{b}}$ & $(27.1)^{\mathrm{b}}$ & $(25.4)^{\mathrm{b}}$ & $(100.0)^{\mathrm{b}}$ \\
\hline \multirow[t]{3}{*}{ No } & 05 & 03 & 02 & & 10 \\
\hline & $(10.2)^{\mathrm{a}}$ & $(15.0)^{\mathrm{a}}$ & $(10.0)^{\mathrm{a}}$ & - & $(8.3)^{\mathrm{a}}$ \\
\hline & $(50.0)^{\mathrm{b}}$ & $(30.0)^{\mathrm{b}}$ & $(20.0)^{b}$ & & $(100.0)^{b}$ \\
\hline \multirow[t]{3}{*}{ Cannot say } & 24 & 16 & 02 & 09 & 51 \\
\hline & $(48.9)^{\mathrm{a}}$ & $(59.2)^{\mathrm{a}}$ & $(10.0)^{\mathrm{a}}$ & $(37.5)^{\mathrm{a}}$ & $(42.5)^{\mathrm{a}}$ \\
\hline & $(47.1)^{\mathrm{b}}$ & $(31.4)^{b}$ & $(3.9)^{\mathrm{b}}$ & $(17.6)^{b}$ & $(100.0)^{b}$ \\
\hline \multirow[t]{3}{*}{$\mathrm{N}$} & 49 & 27 & 20 & 24 & 120 \\
\hline & $(40.8)^{\mathrm{b}}$ & $(22.5)^{\mathrm{b}}$ & $(16.6)^{b}$ & $(20.0)^{\mathrm{b}}$ & $(100.0)^{b}$ \\
\hline & $(100.0)^{\mathrm{a}}$ & $(100.0)^{\mathrm{a}}$ & $(100.0)^{\mathrm{a}}$ & $(100.0)^{\mathrm{a}}$ & $(100.0)^{\mathrm{a}}$ \\
\hline
\end{tabular}

Source: Same as Table 1

Note: Same as Table 1 


\subsection{Infrastructural and banking expectation of youth}

Table 13 reveals thatonly a small proportion of sampled youth was satisfied with drinking water supply (17\%) followed by social environment (15.4\%), power supply (14.2\%), transport facilities (12.6\%), road construction (11.9\%), education $(11.9 \%)$, health $(10.4 \%)$ and public administration $(6.3 \%)$. Table 14 reveals that a significant proportion of them expected an improvement in the health sector $(16.8 \%)$, followed by education infrastructure $(16.5 \%)$, road construction (15.7\%), industrial infrastructure (15.1\%), renewable energy (12.7\%), urban transport (9.6\%), railways $(7.02 \%)$ and airport infrastructure $(6.26 \%)$ with variation across selected districts. Youth of district Jammu expected major improvement in industrial and transportation infrastructure, while the youth of district Kathua wanted a major improvement in the railway infrastructure, whereas, the youth of districts of Rajouri and Poonch expected an improvement in road construction. Table 15 reveals that the majority of sampled youth-rated the availability of banking facilities as good (60.8\%). Major banking constraints included poor availability, lack of transparency, improper time management, bad customer services and loan issues with significant variation across selected districts (see Table 16). Table 17 reveals youth expectations regarding changes in availing banking services in the future focusing on transparency (32.4\%) followed by proper time management (27.2\%), cooperation from banking staff $(24.8 \%)$ and consistency in transactions $(16.4 \%)$.

Table 13. Satisfaction with basic facilities (No./\%)

\begin{tabular}{|c|c|c|c|c|c|}
\hline \multirow{2}{*}{ Facilities } & \multicolumn{5}{|c|}{ Districts } \\
\hline & Jammu & Kathua & Rajouri & Poonch & $\mathrm{N}$ \\
\hline \multirow[t]{3}{*}{ Drinking water facility } & 21 & 21 & 13 & 17 & 67 \\
\hline & $(16.1)^{\mathrm{a}}$ & $(17.7)^{\mathrm{a}}$ & $(17.8)^{\mathrm{a}}$ & $(16.8)^{\mathrm{a}}$ & $(17.0)^{\mathrm{a}}$ \\
\hline & $(31.3)^{\mathrm{b}}$ & $(23.8)^{\mathrm{b}}$ & $(19.4)^{\mathrm{b}}$ & $(25.4)^{\mathrm{b}}$ & $(100.0)^{\mathrm{b}}$ \\
\hline \multirow[t]{3}{*}{ Power supply } & 17 & 13 & 10 & 16 & 56 \\
\hline & $(13.1)^{\mathrm{a}}$ & $(14.4)^{\mathrm{a}}$ & $(13.6)^{\mathrm{a}}$ & $(15.9)^{\mathrm{a}}$ & $(14.2)^{\mathrm{a}}$ \\
\hline & $(30.3)^{\mathrm{b}}$ & $(23.2)^{\mathrm{b}}$ & $(17.8)^{\mathrm{b}}$ & $(28.5)^{\mathrm{b}}$ & $(100.0)^{\mathrm{b}}$ \\
\hline \multirow[t]{3}{*}{ Social environment } & 20 & 15 & 11 & 15 & 61 \\
\hline & $(15.3)^{\mathrm{a}}$ & $(16.6)^{\mathrm{a}}$ & $(15.1)^{\mathrm{a}}$ & $(14.8)^{\mathrm{a}}$ & $(15.4)^{\mathrm{a}}$ \\
\hline & $(32.7)^{\mathrm{b}}$ & $(24.5)^{\mathrm{b}}$ & $(18.0)^{\mathrm{b}}$ & $(24.5)^{\mathrm{b}}$ & $(100.0)^{\mathrm{b}}$ \\
\hline \multirow[t]{3}{*}{ Transport facilities } & 21 & 11 & 09 & 09 & 50 \\
\hline & $(16.1)^{\mathrm{a}}$ & $(12.2)^{\mathrm{a}}$ & $(12.3)^{\mathrm{a}}$ & $(8.9)^{\mathrm{a}}$ & $(12.6)^{\mathrm{a}}$ \\
\hline & $(42.0)^{\mathrm{b}}$ & $(22.0)^{\mathrm{b}}$ & $(18.0)^{\mathrm{b}}$ & $(18.0)^{\mathrm{b}}$ & $(100.0)^{\mathrm{b}}$ \\
\hline \multirow[t]{3}{*}{ Administration } & 09 & 05 & 05 & 06 & 25 \\
\hline & $(6.9)^{\mathrm{a}}$ & $(5.5)^{\mathrm{a}}$ & $(6.8)^{\mathrm{a}}$ & $(5.9)^{\mathrm{a}}$ & $(6.3)^{\mathrm{a}}$ \\
\hline & $(36.0)^{\mathrm{b}}$ & $(20.0)^{\mathrm{b}}$ & $(20.0)^{\mathrm{b}}$ & $(24.0)^{\mathrm{b}}$ & $(100.0)^{\mathrm{b}}$ \\
\hline \multirow[t]{3}{*}{ Road construction } & 17 & 11 & 07 & 12 & 47 \\
\hline & $(13.1)^{\mathrm{a}}$ & $(12.2)^{\mathrm{a}}$ & $(9.5)^{\mathrm{a}}$ & $(11.9)^{\mathrm{a}}$ & $(11.9)^{\mathrm{a}}$ \\
\hline & $(36.2)^{b}$ & $(23.4)^{\mathrm{b}}$ & $(14.8)^{\mathrm{b}}$ & $(25.5)^{\mathrm{b}}$ & $(100.0)^{b}$ \\
\hline \multirow[t]{3}{*}{ Education } & 13 & 11 & 08 & 15 & 47 \\
\hline & $(10.0)^{\mathrm{a}}$ & $(12.2)^{\mathrm{a}}$ & $(10.9)^{\mathrm{a}}$ & $(14.8)^{\mathrm{a}}$ & $(11.9)^{\mathrm{a}}$ \\
\hline & $(27.6)^{\mathrm{b}}$ & $(23.4)^{\mathrm{b}}$ & $(17.0)^{b}$ & $(31.9)^{\mathrm{b}}$ & $(100.0)^{\mathrm{b}}$ \\
\hline \multirow[t]{3}{*}{ Health } & 12 & 08 & 10 & 11 & 41 \\
\hline & $(9.2)^{\mathrm{a}}$ & $(8.8)^{\mathrm{a}}$ & $(13.6)^{\mathrm{a}}$ & $(10.9)^{\mathrm{a}}$ & $(10.4)^{\mathrm{a}}$ \\
\hline & $(29.3)^{b}$ & $(19.5)^{\mathrm{b}}$ & $(24.3)^{\mathrm{b}}$ & $(26.8)^{\mathrm{b}}$ & $(100.0)^{\mathrm{b}}$ \\
\hline \multirow[t]{3}{*}{$\mathrm{N}$} & 130 & 90 & 73 & 101 & 394 \\
\hline & $(32.9)^{\mathrm{b}}$ & $(22.8)^{\mathrm{b}}$ & $(18.5)^{b}$ & $(25.6)^{\mathrm{b}}$ & $(100.0)^{b}$ \\
\hline & $(100.0)^{\mathrm{a}}$ & $(100.0)^{\mathrm{a}}$ & $(100.0)^{\mathrm{a}}$ & $(100.0)^{\mathrm{a}}$ & $(100.0)^{\mathrm{a}}$ \\
\hline
\end{tabular}

Source: Same as Table 1

Note: Same as Table 1 
Table 14. Necessary things to improve infrastructure (No./\%)

\begin{tabular}{|c|c|c|c|c|c|}
\hline \multirow{2}{*}{ Improving infrastructure } & \multicolumn{5}{|c|}{ Districts } \\
\hline & Jammu & Kathua & Rajouri & Poonch & $\mathrm{N}$ \\
\hline \multirow[t]{3}{*}{ Road construction } & 32 & 19 & 15 & 17 & 83 \\
\hline & $(15.2)^{\mathrm{a}}$ & $(15.2)^{\mathrm{a}}$ & $(18.1)^{\mathrm{a}}$ & $(15.6)^{\mathrm{a}}$ & $(15.7)^{\mathrm{a}}$ \\
\hline & $(38.5)^{b}$ & $(22.8)^{\mathrm{b}}$ & $(18.1)^{\mathrm{b}}$ & $(20.5)^{b}$ & $(100.0)^{\mathrm{b}}$ \\
\hline \multirow[t]{3}{*}{ Railways } & 13 & 11 & 05 & 08 & 37 \\
\hline & $(6.1)^{\mathrm{a}}$ & $(8.8)^{\mathrm{a}}$ & $(6.02)^{\mathrm{a}}$ & $(7.3)^{\mathrm{a}}$ & $(7.02)^{\mathrm{a}}$ \\
\hline & $(35.1)^{\mathrm{b}}$ & $(29.7)^{b}$ & $(13.5)^{\mathrm{b}}$ & $(21.6)^{\mathrm{b}}$ & $(100.0)^{b}$ \\
\hline \multirow[t]{3}{*}{ Airport infrastructure } & 13 & 08 & 05 & 07 & 33 \\
\hline & $(6.1)^{\mathrm{a}}$ & $(6.4)^{\mathrm{a}}$ & $(6.02)^{\mathrm{a}}$ & $(6.4)^{\mathrm{a}}$ & $(6.26)^{\mathrm{a}}$ \\
\hline & $(35.1)^{\mathrm{b}}$ & $(21.6)^{b}$ & $(13.5)^{\mathrm{b}}$ & $(18.9)^{\mathrm{b}}$ & $(100.0)^{b}$ \\
\hline \multirow[t]{3}{*}{ Education infrastructure } & 35 & 22 & 13 & 17 & 87 \\
\hline & $(16.6)^{\mathrm{a}}$ & $(17.6)^{\mathrm{a}}$ & $(15.6)^{\mathrm{a}}$ & $(15.5)^{\mathrm{a}}$ & $(16.50)^{\mathrm{a}}$ \\
\hline & $(40.2)^{\mathrm{b}}$ & $(25.3)^{b}$ & $(14.9)^{\mathrm{b}}$ & $(19.5)^{b}$ & $(100.0)^{\mathrm{b}}$ \\
\hline \multirow[t]{3}{*}{ Industrial infrastructure } & 35 & 18 & 11 & 16 & 80 \\
\hline & $(16.6)^{\mathrm{a}}$ & $(14.4)^{\mathrm{a}}$ & $(13.3)^{\mathrm{a}}$ & $(14.6)^{\mathrm{a}}$ & $(15.1)^{\mathrm{a}}$ \\
\hline & $(43.7)^{b}$ & $(22.5)^{b}$ & $(13.7)^{\mathrm{b}}$ & $(20.0)^{\mathrm{b}}$ & $(100.0)^{b}$ \\
\hline \multirow[t]{3}{*}{ Renewable energy } & 26 & 15 & 11 & 15 & 67 \\
\hline & $(12.3)^{\mathrm{a}}$ & $(12.0)^{\mathrm{a}}$ & $(13.3)^{\mathrm{a}}$ & $(13.7)^{\mathrm{a}}$ & $(12.7)^{\mathrm{a}}$ \\
\hline & $(38.8)^{b}$ & $(22.4)^{\mathrm{b}}$ & $(16.4)^{\mathrm{b}}$ & $(22.4)^{b}$ & $(100.0)^{\mathrm{b}}$ \\
\hline \multirow[t]{3}{*}{ Health sector } & 37 & 21 & 15 & 16 & 89 \\
\hline & $(3.33)^{\mathrm{a}}$ & $(16.8)^{\mathrm{a}}$ & $(18.1)^{\mathrm{a}}$ & $(14.6)^{\mathrm{a}}$ & $(16.8)^{\mathrm{a}}$ \\
\hline & $(41.5)^{b}$ & $(23.5)^{\mathrm{b}}$ & $(16.8)^{b}$ & $(17.9)^{\mathrm{b}}$ & $(100.0)^{\mathrm{b}}$ \\
\hline \multirow[t]{3}{*}{ Transportation facilities } & 19 & 11 & 08 & 13 & 51 \\
\hline & $(9.04)^{\mathrm{a}}$ & $(8.8)^{\mathrm{a}}$ & $(9.6)^{\mathrm{a}}$ & $(11.9)^{\mathrm{a}}$ & $(9.6)^{\mathrm{a}}$ \\
\hline & $(37.2)^{b}$ & $(21.5)^{b}$ & $(15.6)^{\mathrm{b}}$ & $(25.5)^{b}$ & $(100.0)^{b}$ \\
\hline \multirow[t]{3}{*}{$\mathrm{N}$} & 210 & 125 & 83 & 109 & 527 \\
\hline & $(39.8)^{\mathrm{b}}$ & $(23.7)^{\mathrm{b}}$ & $(15.7)^{\mathrm{b}}$ & $(20.6)^{\mathrm{b}}$ & $(100.0)^{\mathrm{b}}$ \\
\hline & $(100.0)^{\mathrm{a}}$ & $(100.0)^{\mathrm{a}}$ & $(100.0)^{\mathrm{a}}$ & $(100.0)^{\mathrm{a}}$ & $(100.0)^{\mathrm{a}}$ \\
\hline
\end{tabular}

Source: Same as Table 1

Note: Same as Table 1

Table 15. Impression of banking facilities (No./\%)

\begin{tabular}{|c|c|c|c|c|c|}
\hline \multirow{2}{*}{ Response } & \multicolumn{5}{|c|}{ Districts } \\
\hline & Jammu & Kathua & Rajouri & Poonch & $\mathrm{N}$ \\
\hline \multirow[t]{3}{*}{ Good } & 37 & 13 & 09 & 14 & 73 \\
\hline & $(75.5)^{\mathrm{a}}$ & $(48.1)^{\mathrm{a}}$ & $(45.0)^{\mathrm{a}}$ & $(58.3)^{\mathrm{a}}$ & $(60.8)^{\mathrm{a}}$ \\
\hline & $(50.6)^{\mathrm{b}}$ & $(17.8)^{b}$ & $(12.3)^{b}$ & $(19.2)^{b}$ & $(100.0)^{b}$ \\
\hline \multirow[t]{3}{*}{$\mathrm{Bad}$} & 11 & 10 & 09 & 06 & 36 \\
\hline & $(22.4)^{\mathrm{a}}$ & $(37.03)^{\mathrm{a}}$ & $(45.0)^{\mathrm{a}}$ & $(25.0)^{\mathrm{a}}$ & $(30.0)^{\mathrm{a}}$ \\
\hline & $(30.5)^{b}$ & $(27.7)^{\mathrm{b}}$ & $(25.0)^{\mathrm{b}}$ & $(16.6)^{b}$ & $(100.0)^{b}$ \\
\hline \multirow[t]{3}{*}{ Cannot say } & 01 & 04 & 02 & 04 & 11 \\
\hline & $(2.04)^{\mathrm{a}}$ & $(14.8)^{\mathrm{a}}$ & $(10.0)^{\mathrm{a}}$ & $(16.6)^{\mathrm{a}}$ & $(9.3)^{\mathrm{a}}$ \\
\hline & $(9.1)^{\mathrm{b}}$ & $(36.4)^{\mathrm{b}}$ & $(18.2)^{b}$ & $(36.4)^{b}$ & $(100.0)^{b}$ \\
\hline \multirow[t]{3}{*}{$\mathrm{N}$} & 49 & 27 & 20 & 24 & 120 \\
\hline & $(40.8)^{\mathrm{b}}$ & $(22.5)^{\mathrm{b}}$ & $(16.6)^{b}$ & $(20.0)^{\mathrm{b}}$ & $(100.0)^{b}$ \\
\hline & $(100.0)^{\mathrm{a}}$ & $(100.0)^{\mathrm{a}}$ & $(100.0)^{\mathrm{a}}$ & $(100.0)^{\mathrm{a}}$ & $(100.0)^{\mathrm{a}}$ \\
\hline
\end{tabular}

Source: Same as Table 1

Note: Same as Table 1 
Table 16. Constraints faced in availing banking services (No./\%)

\begin{tabular}{|c|c|c|c|c|c|}
\hline \multirow{2}{*}{ Constraints } & \multicolumn{5}{|c|}{ Districts } \\
\hline & Jammu & Kathua & Rajouri & Poonch & $\mathrm{N}$ \\
\hline \multirow[t]{3}{*}{ Poor availability of services } & 07 & 03 & 04 & 04 & 18 \\
\hline & $(33.3)^{\mathrm{a}}$ & $(10.0)^{\mathrm{a}}$ & $(18.1)^{\mathrm{a}}$ & $(14.8)^{\mathrm{a}}$ & $(18.0)^{\mathrm{a}}$ \\
\hline & $(38.8)^{\mathrm{b}}$ & $(16.6)^{b}$ & $(22.2)^{\mathrm{b}}$ & $(22.2)^{\mathrm{b}}$ & $(100.0)^{\mathrm{b}}$ \\
\hline \multirow[t]{3}{*}{ Lack of consistency } & 04 & 03 & 01 & 04 & 12 \\
\hline & $(19.04)^{\mathrm{a}}$ & $(10.0)^{\mathrm{a}}$ & $(4.5)^{\mathrm{a}}$ & $(14.8)^{\mathrm{a}}$ & $(12.0)^{\mathrm{a}}$ \\
\hline & $(33.3)^{b}$ & $(25.0)^{\mathrm{b}}$ & $(8.3)^{\mathrm{b}}$ & $(33.3)^{\mathrm{b}}$ & $(100.0)^{b}$ \\
\hline \multirow[t]{3}{*}{ Lack of transparency } & 03 & 05 & 04 & 04 & 16 \\
\hline & $(14.2)^{\mathrm{a}}$ & $(16.6)^{\mathrm{a}}$ & $(18.1)^{\mathrm{a}}$ & $(14.8)^{\mathrm{a}}$ & $(16.3)^{\mathrm{a}}$ \\
\hline & $(18.7)^{\mathrm{b}}$ & $(31.2)^{\mathrm{b}}$ & $(25.0)^{\mathrm{b}}$ & $(25.0)^{\mathrm{b}}$ & $(100.0)^{b}$ \\
\hline \multirow[t]{3}{*}{ Improper time management } & 03 & 05 & 05 & 05 & 18 \\
\hline & $(14.2)^{\mathrm{a}}$ & $(16.6)^{\mathrm{a}}$ & $(22.7)^{\mathrm{a}}$ & $(18.5)^{\mathrm{a}}$ & $(18.0)^{\mathrm{a}}$ \\
\hline & $(16.6)^{b}$ & $(27.7)^{b}$ & $(27.7)^{b}$ & $(27.7)^{b}$ & $(100.0)^{\mathrm{b}}$ \\
\hline \multirow[t]{3}{*}{ Large over dues } & & 03 & 03 & 03 & 09 \\
\hline & - & $(10.0)^{\mathrm{a}}$ & $(13.6)^{\mathrm{a}}$ & $(11.1)^{\mathrm{a}}$ & $(9.0)^{\mathrm{a}}$ \\
\hline & & $(33.3)^{\mathrm{b}}$ & $(33.3)^{\mathrm{b}}$ & $(33.3)^{\mathrm{b}}$ & $(100.0)^{\mathrm{b}}$ \\
\hline \multirow[t]{3}{*}{ Bad customer service } & 02 & 05 & 04 & 02 & 13 \\
\hline & $(9.5)^{\mathrm{a}}$ & $(16.6)^{\mathrm{a}}$ & $(18.1)^{\mathrm{a}}$ & $(7.40)^{\mathrm{a}}$ & $(13.0)^{\mathrm{a}}$ \\
\hline & $(15.4)^{b}$ & $(38.5)^{\mathrm{b}}$ & $(30.7)^{b}$ & $(15.4)^{\mathrm{b}}$ & $(100.0)^{b}$ \\
\hline \multirow[t]{3}{*}{ Loan issues } & 02 & 06 & 01 & 05 & 14 \\
\hline & $(9.5)^{\mathrm{a}}$ & $(20.0)^{\mathrm{a}}$ & $(4.5)^{\mathrm{a}}$ & $(18.5)^{\mathrm{a}}$ & $(14.0)^{\mathrm{a}}$ \\
\hline & $(14.3)^{b}$ & $(42.8)^{b}$ & $(7.2)^{\mathrm{b}}$ & $(35.7)^{b}$ & $(100.0)^{b}$ \\
\hline \multirow[t]{3}{*}{$\mathrm{N}$} & 21 & 30 & 22 & 27 & 100 \\
\hline & $(21.0)^{\mathrm{b}}$ & $(30.0)^{\mathrm{b}}$ & $(22.0)^{\mathrm{b}}$ & $(27.0)^{\mathrm{b}}$ & $(100.0)^{b}$ \\
\hline & $(100.0)^{\mathrm{a}}$ & $(100.0)^{\mathrm{a}}$ & $(100.0)^{\mathrm{a}}$ & $(100.0)^{\mathrm{a}}$ & $(100.0)^{\mathrm{a}}$ \\
\hline
\end{tabular}

Source: Same as Table 1

Note: Same as Table 1

Table 17. Expectations regarding banking services in near future (No./\%)

\begin{tabular}{|c|c|c|c|c|c|}
\hline \multirow{2}{*}{ Changes } & \multicolumn{5}{|c|}{ Districts } \\
\hline & Jammu & Kathua & Rajouri & Poonch & $\mathrm{N}$ \\
\hline \multirow[t]{3}{*}{ Transparency } & 35 & 15 & 15 & 16 & 81 \\
\hline & $(37.2)^{\mathrm{a}}$ & $(27.3)^{\mathrm{a}}$ & $(32.6)^{a}$ & $(28.1)^{\mathrm{a}}$ & $(32.4)^{\mathrm{a}}$ \\
\hline & $(43.2)^{\mathrm{b}}$ & $(18.5)^{\mathrm{b}}$ & $(18.5)^{b}$ & $(19.7)^{\mathrm{b}}$ & $(100.0)^{\mathrm{b}}$ \\
\hline \multirow[t]{3}{*}{ Consistency in transactions } & 11 & 09 & 10 & 11 & 41 \\
\hline & $(11.7)^{\mathrm{a}}$ & $(16.4)^{\mathrm{a}}$ & $(21.7)^{\mathrm{a}}$ & $(19.3)^{\mathrm{a}}$ & $(16.4)^{\mathrm{a}}$ \\
\hline & $(26.8)^{\mathrm{b}}$ & $(21.9)^{\mathrm{b}}$ & $(24.4)^{\mathrm{b}}$ & $(26.8)^{\mathrm{b}}$ & $(100.0)^{\mathrm{b}}$ \\
\hline \multirow[t]{3}{*}{ Proper time management } & 25 & 19 & 09 & 15 & 68 \\
\hline & $(26.5)^{\mathrm{a}}$ & $(34.5)^{\mathrm{a}}$ & $(19.5)^{\mathrm{a}}$ & $(26.3)^{\mathrm{a}}$ & $(27.2)^{\mathrm{a}}$ \\
\hline & $(36.7)^{b}$ & $(27.9)^{\mathrm{b}}$ & $(13.2)^{\mathrm{b}}$ & $(22.1)^{\mathrm{b}}$ & $(100.0)^{\mathrm{b}}$ \\
\hline \multirow[t]{3}{*}{ Cooperation from banking staff } & 23 & 12 & 12 & 15 & 62 \\
\hline & $(24.4)^{\mathrm{a}}$ & $(21.8)^{\mathrm{a}}$ & $(26.1)^{\mathrm{a}}$ & $(26.3)^{\mathrm{a}}$ & $(24.8)^{\mathrm{a}}$ \\
\hline & $(37.1)^{\mathrm{b}}$ & $(19.3)^{\mathrm{b}}$ & $(19.3)^{b}$ & $(24.2)^{\mathrm{b}}$ & $(100.0)^{\mathrm{b}}$ \\
\hline \multirow[t]{3}{*}{$\mathrm{N}$} & 94 & 55 & 46 & 57 & 252 \\
\hline & $(37.3)^{\mathrm{b}}$ & $(21.8)^{\mathrm{b}}$ & $(18.3)^{\mathrm{b}}$ & $(22.6)^{\mathrm{b}}$ & $(100.0)^{\mathrm{b}}$ \\
\hline & $(100.0)^{\mathrm{a}}$ & $(100.0)^{\mathrm{a}}$ & $(100.0)^{\mathrm{a}}$ & $(100.0)^{\mathrm{a}}$ & $(100.0)^{\mathrm{a}}$ \\
\hline
\end{tabular}

Source: Same as Table 1

Note: Same as Table 1 


\subsection{Expectations of economic performance}

Table 18. Rating of Jammu and Kashmir as a poor state (No./\%)

\begin{tabular}{|c|c|c|c|c|c|}
\hline \multirow{2}{*}{ Response } & \multicolumn{5}{|c|}{ Districts } \\
\hline & Jammu & Kathua & Rajouri & Poonch & $\mathrm{N}$ \\
\hline Yes & $\begin{array}{c}16 \\
(32.6)^{\mathrm{a}} \\
(38.1)^{\mathrm{b}}\end{array}$ & $\begin{array}{c}11 \\
(40.7)^{\mathrm{a}} \\
(26.2)^{\mathrm{b}}\end{array}$ & $\begin{array}{c}08 \\
(40.0)^{\mathrm{a}} \\
(19.0)^{\mathrm{b}}\end{array}$ & $\begin{array}{c}07 \\
(29.7)^{\mathrm{a}} \\
(16.6)^{\mathrm{b}}\end{array}$ & $\begin{array}{c}42 \\
(35.0)^{\mathrm{a}} \\
(100.0)^{\mathrm{b}}\end{array}$ \\
\hline No & $\begin{array}{c}27 \\
(56.1)^{\mathrm{a}} \\
(47.4)^{\mathrm{b}}\end{array}$ & $\begin{array}{c}11 \\
(40.7)^{\mathrm{a}} \\
(19.3)^{\mathrm{b}}\end{array}$ & $\begin{array}{c}08 \\
(40.0)^{\mathrm{a}} \\
(14.0)^{\mathrm{b}}\end{array}$ & $\begin{array}{c}11 \\
(45.8)^{\mathrm{a}} \\
(19.3)^{\mathrm{b}}\end{array}$ & $\begin{array}{c}57 \\
(47.5)^{\mathrm{a}} \\
(100.0)^{\mathrm{b}}\end{array}$ \\
\hline Cannot say & $\begin{array}{c}06 \\
(0.6)^{\mathrm{a}} \\
(28.5)^{\mathrm{b}}\end{array}$ & $\begin{array}{c}05 \\
(18.5)^{\mathrm{a}} \\
(23.3)^{\mathrm{b}}\end{array}$ & $\begin{array}{c}04 \\
(20.0)^{\mathrm{a}} \\
(19.0)^{\mathrm{b}}\end{array}$ & $\begin{array}{c}06 \\
(25.0)^{\mathrm{a}} \\
(28.5)^{\mathrm{b}}\end{array}$ & $\begin{array}{c}21 \\
(17.5)^{\mathrm{a}} \\
(100.0)^{\mathrm{b}}\end{array}$ \\
\hline $\mathrm{N}$ & $\begin{array}{c}49 \\
(40.8)^{\mathrm{b}} \\
(100.0)^{\mathrm{a}}\end{array}$ & $\begin{array}{c}27 \\
(22.5)^{\mathrm{b}} \\
(100.0)^{\mathrm{a}}\end{array}$ & $\begin{array}{c}20 \\
(16.6)^{\mathrm{b}} \\
(100.0)^{\mathrm{a}}\end{array}$ & $\begin{array}{c}24 \\
(20.0)^{\mathrm{b}} \\
(100.0)^{\mathrm{a}}\end{array}$ & $\begin{array}{c}120 \\
(100.0)^{\mathrm{b}} \\
(100.0)^{\mathrm{a}}\end{array}$ \\
\hline
\end{tabular}

Source: Same as Table 1

Note: Same as Table 1

Table 19. Ways to reduce poverty in near future (No./\%)

\begin{tabular}{|c|c|c|c|c|c|}
\hline \multirow{2}{*}{ Ways } & \multicolumn{5}{|c|}{ Districts } \\
\hline & Jammu & Kathua & Rajouri & Poonch & $\mathrm{N}$ \\
\hline Literacy & $\begin{array}{c}10 \\
(11.2)^{\mathrm{a}} \\
(41.6)^{\mathrm{b}}\end{array}$ & $\begin{array}{c}04 \\
(7.4)^{\mathrm{a}} \\
(16.6)^{\mathrm{b}}\end{array}$ & $\begin{array}{c}05 \\
(11.9)^{\mathrm{a}} \\
(20.8)^{\mathrm{b}}\end{array}$ & $\begin{array}{c}05 \\
(13.8)^{\mathrm{a}} \\
(20.8)^{\mathrm{b}}\end{array}$ & $\begin{array}{c}24 \\
(20.0)^{\mathrm{a}} \\
(100.0)^{\mathrm{b}}\end{array}$ \\
\hline Training & $\begin{array}{c}08 \\
(8.9)^{\mathrm{a}} \\
(42.1)^{\mathrm{b}}\end{array}$ & $\begin{array}{c}04 \\
(7.4)^{\mathrm{a}} \\
(21.0)^{\mathrm{b}}\end{array}$ & $\begin{array}{c}02 \\
(4.7)^{\mathrm{a}} \\
(10.5)^{\mathrm{b}}\end{array}$ & $\begin{array}{c}05 \\
(13.8)^{\mathrm{a}} \\
(26.3)^{\mathrm{b}}\end{array}$ & $\begin{array}{c}19 \\
(15.8)^{\mathrm{a}} \\
(100.0)^{\mathrm{b}}\end{array}$ \\
\hline Education & $\begin{array}{c}09 \\
(10.1)^{\mathrm{a}} \\
(33.3)^{\mathrm{b}}\end{array}$ & $\begin{array}{c}07 \\
(12.9)^{\mathrm{a}} \\
(25.9)^{\mathrm{b}}\end{array}$ & $\begin{array}{c}06 \\
(14.2)^{\mathrm{a}} \\
(22.2)^{\mathrm{b}}\end{array}$ & $\begin{array}{c}05 \\
(13.8)^{\mathrm{a}} \\
(18.5)^{\mathrm{b}}\end{array}$ & $\begin{array}{c}27 \\
(22.5)^{\mathrm{a}} \\
(100.0)^{\mathrm{b}}\end{array}$ \\
\hline Housing facilities & $\begin{array}{c}08 \\
(8.9)^{\mathrm{a}} \\
(44.4)^{\mathrm{b}}\end{array}$ & $\begin{array}{c}05 \\
(9.2)^{\mathrm{a}} \\
(27.7)^{\mathrm{b}}\end{array}$ & $\begin{array}{c}02 \\
(4.7)^{\mathrm{a}} \\
(11.1)^{\mathrm{b}}\end{array}$ & $\begin{array}{c}03 \\
(8.3)^{\mathrm{a}} \\
(16.6)^{\mathrm{b}}\end{array}$ & $\begin{array}{c}18 \\
(15.0)^{\mathrm{a}} \\
(100.0)^{\mathrm{b}}\end{array}$ \\
\hline Health facilities & $\begin{array}{c}10 \\
(11.2)^{\mathrm{a}} \\
(38.4)^{\mathrm{b}}\end{array}$ & $\begin{array}{c}07 \\
(12.9)^{\mathrm{a}} \\
(26.9)^{\mathrm{b}}\end{array}$ & $\begin{array}{c}04 \\
(9.5)^{\mathrm{a}} \\
(15.4)^{\mathrm{b}}\end{array}$ & $\begin{array}{c}05 \\
(13.8)^{\mathrm{a}} \\
(19.2)^{\mathrm{b}}\end{array}$ & $\begin{array}{c}26 \\
(21.7)^{\mathrm{a}} \\
(100.0)^{\mathrm{b}}\end{array}$ \\
\hline Income and employment supporter & $\begin{array}{c}12 \\
(13.4)^{\mathrm{a}} \\
(42.8)^{\mathrm{b}}\end{array}$ & $\begin{array}{c}08 \\
(14.8)^{\mathrm{a}} \\
(28.5)^{\mathrm{b}}\end{array}$ & $\begin{array}{c}06 \\
(14.2)^{\mathrm{a}} \\
(21.4)^{\mathrm{b}}\end{array}$ & $\begin{array}{c}02 \\
(5.5)^{\mathrm{a}} \\
(7.1)^{\mathrm{b}}\end{array}$ & $\begin{array}{c}28 \\
(23.3)^{\mathrm{a}} \\
(100.0)^{\mathrm{b}}\end{array}$ \\
\hline Create jobs & $\begin{array}{c}11 \\
(12.3)^{\mathrm{a}} \\
(35.4)^{\mathrm{b}}\end{array}$ & $\begin{array}{c}08 \\
(14.8)^{\mathrm{a}} \\
(25.8)^{\mathrm{b}}\end{array}$ & $\begin{array}{c}05 \\
(11.9)^{\mathrm{a}} \\
(16.1)^{\mathrm{b}}\end{array}$ & $\begin{array}{c}07 \\
(19.4)^{\mathrm{a}} \\
(22.5)^{\mathrm{b}}\end{array}$ & $\begin{array}{c}31 \\
(25.8)^{\mathrm{a}} \\
(100.0)^{\mathrm{b}}\end{array}$ \\
\hline Access to credit & $\begin{array}{c}06 \\
(6.7)^{\mathrm{a}} \\
(37.5)^{\mathrm{b}}\end{array}$ & $\begin{array}{c}05 \\
(9.2)^{\mathrm{a}} \\
(31.2)^{\mathrm{b}}\end{array}$ & $\begin{array}{c}04 \\
(4.7)^{\mathrm{a}} \\
(25.0)^{\mathrm{b}}\end{array}$ & $\begin{array}{c}01 \\
(2.7)^{\mathrm{a}} \\
(6.2)^{\mathrm{b}}\end{array}$ & $\begin{array}{c}16 \\
(13.3)^{\mathrm{a}} \\
(100.0)^{\mathrm{b}}\end{array}$ \\
\hline Provide subsidies & $\begin{array}{c}09 \\
(10.1)^{\mathrm{a}} \\
(56.2)^{\mathrm{b}}\end{array}$ & $\begin{array}{c}02 \\
(3.7)^{\mathrm{a}} \\
(12.5)^{\mathrm{b}}\end{array}$ & $\begin{array}{c}04 \\
(4.7)^{\mathrm{a}} \\
(25.0)^{\mathrm{b}}\end{array}$ & $\begin{array}{c}01 \\
(2.7)^{\mathrm{a}} \\
(6.25)^{\mathrm{b}}\end{array}$ & $\begin{array}{c}16 \\
(13.3)^{\mathrm{a}} \\
(100.0)^{\mathrm{b}}\end{array}$ \\
\hline Minimum wage laws & $\begin{array}{c}06 \\
(6.7)^{\mathrm{a}} \\
(37.5)^{\mathrm{b}}\end{array}$ & $\begin{array}{c}04 \\
(7.4)^{\mathrm{a}} \\
(25.0)^{\mathrm{b}}\end{array}$ & $\begin{array}{c}04 \\
(4.7)^{\mathrm{a}} \\
(25.0)^{\mathrm{b}}\end{array}$ & $\begin{array}{c}02 \\
(5.5)^{\mathrm{a}} \\
(12.5)^{\mathrm{b}}\end{array}$ & $\begin{array}{c}16 \\
(13.3)^{\mathrm{a}} \\
(100.0)^{\mathrm{b}}\end{array}$ \\
\hline $\mathrm{N}$ & $\begin{array}{c}89 \\
(40.3)^{\mathrm{b}} \\
(100.0)^{\mathrm{a}}\end{array}$ & $\begin{array}{c}54 \\
(24.4)^{\mathrm{b}} \\
(100.0)^{\mathrm{a}}\end{array}$ & $\begin{array}{c}42 \\
(19.0)^{\mathrm{b}} \\
(100.0)^{\mathrm{a}}\end{array}$ & $\begin{array}{c}36 \\
(16.3)^{\mathrm{b}} \\
(100.0)^{\mathrm{a}}\end{array}$ & $\begin{array}{c}221 \\
(100.0)^{\mathrm{b}} \\
(100.0)^{\mathrm{a}}\end{array}$ \\
\hline
\end{tabular}

Source: Same as Table

Note: Same as Table 1 
Table 18 reveals that more than one-third of sampled youth-rated Jammu and Kashmir as a poor state while $47.5 \%$ rated otherwise and $17.5 \%$ of them were indifferent. Poverty remained the main cause of low economic growth, which needs to be addressed through better education and training, housing, health, greater income and employment support, access to credit, subsidies and better minimum wages (see Table 19). The majority of sampled youth (82.5\%) reported Jammu and Kashmir as the most corrupt (see Table 20). The Article 370 was the root cause of corruption and the biggest hurdle for industrial development and other development projects in Jammu and Kashmir. Table 21 reveals that sampled youth expected to reduce corruption in the future through greater transparency (34.5\%) followed by empowering citizens $(21.01 \%)$, strong punitive measures $(16.2 \%)$, reducing horizontal inequalities $(36.7 \%)$ and reforming finance management (13.2\%).

Table 20. Rating of Jammu and Kashmir as a corrupt State (No./\%)

\begin{tabular}{|c|c|c|c|c|c|}
\hline \multirow{2}{*}{ Response } & \multicolumn{5}{|c|}{ Districts } \\
\hline & Jammu & Kathua & Rajouri & Poonch & $\mathrm{N}$ \\
\hline \multirow[t]{3}{*}{ Yes } & 43 & 22 & 16 & 18 & 99 \\
\hline & $(87.7)^{\mathrm{a}}$ & $(81.4)^{\mathrm{a}}$ & $(80.0)^{\mathrm{a}}$ & $(75.0)^{\mathrm{a}}$ & $(82.5)^{a}$ \\
\hline & $(43.4)^{b}$ & $(22.2)^{\mathrm{b}}$ & $(16.2)^{b}$ & $(18.2)^{b}$ & $(100.0)^{b}$ \\
\hline \multirow[t]{3}{*}{ No } & 06 & 05 & 04 & 06 & 21 \\
\hline & $(12.3)^{\mathrm{a}}$ & $(18.5)^{\mathrm{a}}$ & $(20.0)^{\mathrm{a}}$ & $(25.0)^{\mathrm{a}}$ & $(17.5)^{\mathrm{a}}$ \\
\hline & $(28.5)^{\mathrm{b}}$ & $(23.8)^{\mathrm{b}}$ & $(19.0)^{\mathrm{b}}$ & $(28.5)^{\mathrm{b}}$ & $(100.0)^{\mathrm{b}}$ \\
\hline \multirow[t]{3}{*}{$\mathrm{N}$} & 49 & 27 & 20 & 24 & 120 \\
\hline & $(40.8)^{\mathrm{b}}$ & $(22.5)^{\mathrm{b}}$ & $(16.6)^{\mathrm{b}}$ & $(20.0)^{\mathrm{b}}$ & $(100.0)^{\mathrm{b}}$ \\
\hline & $(100.0)^{\mathrm{a}}$ & $(100.0)^{\mathrm{a}}$ & $(100.0)^{\mathrm{a}}$ & $(100.0)^{\mathrm{a}}$ & $(100.0)^{\mathrm{a}}$ \\
\hline
\end{tabular}

Source: Same as Table 1

Note: Same as Table 1

Table 21. Ways to reduce corruption in Jammu and Kashmir (No./\%)

\begin{tabular}{|c|c|c|c|c|c|}
\hline \multirow{2}{*}{ Ways } & \multicolumn{5}{|c|}{ Districts } \\
\hline & Jammu & Kathua & Rajouri & Poonch & $\mathrm{N}$ \\
\hline \multirow[t]{3}{*}{ Transparency } & 42 & 23 & 17 & 20 & 102 \\
\hline & $(35.0)^{\mathrm{a}}$ & $(35.3)^{\mathrm{a}}$ & $(33.3)^{\mathrm{a}}$ & $(33.8)^{\mathrm{a}}$ & $(34.5)^{\mathrm{a}}$ \\
\hline & $(41.2)^{\mathrm{b}}$ & $(22.5)^{\mathrm{b}}$ & $(16.6)^{b}$ & $(19.6)^{b}$ & $(100.0)^{b}$ \\
\hline \multirow[t]{3}{*}{ Empower citizens } & 24 & 15 & 10 & 13 & 62 \\
\hline & $(20.0)^{\mathrm{a}}$ & $(23.1)^{\mathrm{a}}$ & $(19.6)^{\mathrm{a}}$ & $(22.03)^{\mathrm{a}}$ & $(21.01)^{\mathrm{a}}$ \\
\hline & $(38.7)^{\mathrm{b}}$ & $(24.2)^{\mathrm{b}}$ & $(16.1)^{\mathrm{b}}$ & $(20.9)^{\mathrm{b}}$ & $(100.0)^{\mathrm{b}}$ \\
\hline \multirow[t]{3}{*}{ Reform finance management } & 16 & 12 & 06 & 05 & 39 \\
\hline & $(13.3)^{\mathrm{a}}$ & $(18.4)^{\mathrm{a}}$ & $(11.7)^{\mathrm{a}}$ & $(8.4)^{\mathrm{a}}$ & $(13.2)^{\mathrm{a}}$ \\
\hline & $(41.0)^{\mathrm{b}}$ & $(30.7)^{\mathrm{b}}$ & $(15.4)^{b}$ & $(12.8)^{\mathrm{b}}$ & $(100.0)^{b}$ \\
\hline \multirow[t]{3}{*}{ Strong punitive measures } & 22 & 08 & 09 & 09 & 48 \\
\hline & $(18.3)^{\mathrm{a}}$ & $(12.3)^{\mathrm{a}}$ & $(17.6)^{\mathrm{a}}$ & $(15.2)^{\mathrm{a}}$ & $(16.2)^{\mathrm{a}}$ \\
\hline & $(45.8)^{\mathrm{b}}$ & $(16.6)^{b}$ & $(18.7)^{b}$ & $(18.7)^{b}$ & $(100.0)^{b}$ \\
\hline \multirow[t]{3}{*}{ Reducing horizontal inequalities } & 16 & 07 & 09 & 12 & 44 \\
\hline & $(13.3)^{\mathrm{a}}$ & $(10.7)^{\mathrm{a}}$ & $(17.6)^{\mathrm{a}}$ & $(20.3)^{\mathrm{a}}$ & $(36.7)^{\mathrm{a}}$ \\
\hline & $(36.4)^{\mathrm{b}}$ & $(15.9)^{\mathrm{b}}$ & $(20.4)^{b}$ & $(27.3)^{\mathrm{b}}$ & $(100.0)^{b}$ \\
\hline \multirow[t]{3}{*}{$\mathrm{N}$} & 120 & 65 & 51 & 59 & 295 \\
\hline & $(40.6)^{\mathrm{b}}$ & $(22.0)^{\mathrm{b}}$ & $(17.3)^{\mathrm{b}}$ & $(20.0)^{\mathrm{b}}$ & $(100.0)^{b}$ \\
\hline & $(100.0)^{\mathrm{a}}$ & $(100.0)^{\mathrm{a}}$ & $(100.0)^{\mathrm{a}}$ & $(100.0)^{\mathrm{a}}$ & $(100.0)^{\mathrm{a}}$ \\
\hline
\end{tabular}

Source: Same as Table 1

Note: Same as Table 1 


\subsection{Expectations of youth violence}

Table 22. Rating of youth involvement in violence (No./\%)

\begin{tabular}{cccccc}
\hline \multirow{2}{*}{ Response } & \multicolumn{5}{c}{ Districts } \\
\cline { 2 - 6 } & Jammu & Kathua & Rajouri & Poonch & $\mathrm{N}$ \\
\hline Yes & 40 & 15 & 13 & 13 & 81 \\
& $(81.6)^{\mathrm{a}}$ & $(55.5)^{\mathrm{a}}$ & $(65.0)^{\mathrm{a}}$ & $(54.7)^{\mathrm{a}}$ & $(67.5)^{\mathrm{a}}$ \\
& $(49.4)^{\mathrm{b}}$ & $(18.5)^{\mathrm{b}}$ & $(16.0)^{\mathrm{b}}$ & $(16.0)^{\mathrm{b}}$ & $(100.0)^{\mathrm{b}}$ \\
No & 04 & 06 & 06 & 03 & 19 \\
& $(8.7)^{\mathrm{a}}$ & $(22.20)^{\mathrm{a}}$ & $(30.0)^{\mathrm{a}}$ & $(12.5)^{\mathrm{a}}$ & $(15.8)^{\mathrm{a}}$ \\
& $(21.1)^{\mathrm{b}}$ & $(31.5)^{\mathrm{b}}$ & $(31.5)^{\mathrm{b}}$ & $(15.7)^{\mathrm{b}}$ & $(100.0)^{\mathrm{b}}$ \\
Cannot say & 05 & 06 & 01 & 08 & 20 \\
& $(10.2)^{\mathrm{a}}$ & $(22.2)^{\mathrm{a}}$ & $(5.0)^{\mathrm{a}}$ & $(33.3)^{\mathrm{a}}$ & $(16.7)^{\mathrm{a}}$ \\
& $(25.0)^{\mathrm{b}}$ & $(30.0)^{\mathrm{b}}$ & $(5.0)^{\mathrm{b}}$ & $(40.0)^{\mathrm{b}}$ & $(100.0)^{\mathrm{b}}$ \\
$\mathrm{N}$ & 49 & 27 & 20 & 24 & 120 \\
& $(40.8)^{\mathrm{b}}$ & $(22.5)^{\mathrm{b}}$ & $(16.6)^{\mathrm{b}}$ & $(20.0)^{\mathrm{b}}$ & $(100.0)^{\mathrm{b}}$ \\
& $(100.0)^{\mathrm{a}}$ & $(100.0)^{\mathrm{a}}$ & $(100.0)^{\mathrm{a}}$ & $(100.0)^{\mathrm{a}}$ & $(100.0)^{\mathrm{a}}$ \\
\hline
\end{tabular}

Source: Same as Table 1

Note: Same as Table 1

Table 23. Reasons for youth involvement in violence (No./\%)

\begin{tabular}{|c|c|c|c|c|c|}
\hline \multirow{2}{*}{ Reasons } & \multicolumn{5}{|c|}{ Districts } \\
\hline & Jammu & Kathua & Rajouri & Poonch & $\mathrm{N}$ \\
\hline Widespread corruption & $\begin{array}{c}28 \\
(19.5)^{\mathrm{a}} \\
(48.3)^{\mathrm{b}}\end{array}$ & $\begin{array}{c}11 \\
(15.7)^{\mathrm{a}} \\
(18.9)^{\mathrm{b}}\end{array}$ & $\begin{array}{c}08 \\
(22.8)^{\mathrm{a}} \\
(13.7)^{\mathrm{b}}\end{array}$ & $\begin{array}{c}11 \\
(21.2)^{\mathrm{a}} \\
(18.9)^{\mathrm{b}}\end{array}$ & $\begin{array}{c}58 \\
(15.7)^{\mathrm{a}} \\
(100.0)^{\mathrm{b}}\end{array}$ \\
\hline Unequal access to relief & $\begin{array}{c}04 \\
(2.7)^{\mathrm{a}} \\
(33.3)^{\mathrm{b}}\end{array}$ & $\begin{array}{c}03 \\
(4.2)^{\mathrm{a}} \\
(25.0)^{\mathrm{b}}\end{array}$ & - & $\begin{array}{c}05 \\
(9.6)^{\mathrm{a}} \\
(41.6)^{\mathrm{b}}\end{array}$ & $\begin{array}{c}12 \\
(3.9)^{\mathrm{a}} \\
(100.0)^{\mathrm{b}}\end{array}$ \\
\hline $\begin{array}{c}\text { Unequal access to } \\
\text { developmental assistance }\end{array}$ & $\begin{array}{c}06 \\
(4.1)^{\mathrm{a}} \\
(40.0)^{\mathrm{b}}\end{array}$ & $\begin{array}{c}03 \\
(4.2)^{\mathrm{a}} \\
(20.0)^{\mathrm{b}}\end{array}$ & $\begin{array}{c}01 \\
(2.8)^{\mathrm{a}} \\
(6.6)^{\mathrm{b}}\end{array}$ & $\begin{array}{c}05 \\
(9.6)^{\mathrm{a}} \\
(33.3)^{\mathrm{b}}\end{array}$ & $\begin{array}{c}15 \\
(4.9)^{\mathrm{a}} \\
(100.0)^{\mathrm{b}}\end{array}$ \\
\hline Exclusion in education & $\begin{array}{c}15 \\
(10.5)^{\mathrm{a}} \\
(45.4)^{\mathrm{b}}\end{array}$ & $\begin{array}{c}09 \\
(12.8)^{\mathrm{a}} \\
(27.3)^{\mathrm{b}}\end{array}$ & $\begin{array}{c}03 \\
(8.6)^{\mathrm{a}} \\
(9.1)^{\mathrm{b}}\end{array}$ & $\begin{array}{c}06 \\
(11.5)^{\mathrm{a}} \\
(18.2)^{\mathrm{b}}\end{array}$ & $\begin{array}{c}33 \\
(10.9)^{\mathrm{a}} \\
(100.0)^{\mathrm{b}}\end{array}$ \\
\hline Exclusion in employment & $\begin{array}{c}20 \\
(13.9)^{\mathrm{a}} \\
(46.5)^{\mathrm{b}}\end{array}$ & $\begin{array}{c}11 \\
(15.7)^{\mathrm{a}} \\
(25.5)^{\mathrm{b}}\end{array}$ & $\begin{array}{c}07 \\
(20.0)^{\mathrm{a}} \\
(16.3)^{\mathrm{b}}\end{array}$ & $\begin{array}{c}05 \\
(9.6)^{\mathrm{a}} \\
(11.6)^{\mathrm{b}}\end{array}$ & $\begin{array}{c}43 \\
(14.2)^{\mathrm{a}} \\
(100.0)^{\mathrm{b}}\end{array}$ \\
\hline Unequal access to social services & $\begin{array}{c}14 \\
(9.7)^{\mathrm{a}} \\
(58.3)^{\mathrm{b}}\end{array}$ & $\begin{array}{c}02 \\
(2.8)^{\mathrm{a}} \\
(8.3)^{\mathrm{b}}\end{array}$ & $\begin{array}{c}04 \\
(11.4)^{\mathrm{a}} \\
(16.6)^{\mathrm{b}}\end{array}$ & $\begin{array}{c}04 \\
(7.6)^{\mathrm{a}} \\
(16.6)^{\mathrm{b}}\end{array}$ & $\begin{array}{c}24 \\
(7.9)^{\mathrm{a}} \\
(100.0)^{\mathrm{b}}\end{array}$ \\
\hline Lack of jobs & $\begin{array}{c}26 \\
(18.1)^{\mathrm{a}} \\
(44.0)^{\mathrm{b}}\end{array}$ & $\begin{array}{c}16 \\
(22.8)^{\mathrm{a}} \\
(27.1)^{\mathrm{b}}\end{array}$ & $\begin{array}{c}10 \\
(28.5)^{\mathrm{a}} \\
(16.9)^{\mathrm{b}}\end{array}$ & $\begin{array}{c}07 \\
(13.5)^{\mathrm{a}} \\
(11.8)^{\mathrm{b}}\end{array}$ & $\begin{array}{c}59 \\
(19.5)^{\mathrm{a}} \\
(100.0)^{\mathrm{b}}\end{array}$ \\
\hline $\begin{array}{l}\text { Restriction on cultural } \\
\text { and social practices }\end{array}$ & $\begin{array}{c}13 \\
(9.0)^{\mathrm{a}} \\
(56.5)^{\mathrm{b}}\end{array}$ & $\begin{array}{c}05 \\
(7.1)^{\mathrm{a}} \\
(21.7)^{\mathrm{b}}\end{array}$ & $\begin{array}{c}02 \\
(5.7)^{\mathrm{a}} \\
(8.6)^{\mathrm{b}}\end{array}$ & $\begin{array}{c}03 \\
(5.7)^{\mathrm{a}} \\
(13.0)^{\mathrm{b}}\end{array}$ & $\begin{array}{c}23 \\
(7.6)^{\mathrm{a}} \\
(100.0)^{\mathrm{b}}\end{array}$ \\
\hline Economic security & $\begin{array}{c}17 \\
(11.8)^{\mathrm{a}} \\
(48.5)^{\mathrm{b}}\end{array}$ & $\begin{array}{c}10 \\
(14.3)^{\mathrm{a}} \\
(28.5)^{\mathrm{b}}\end{array}$ & $\begin{array}{c}02 \\
(5.7)^{\mathrm{a}} \\
(5.7)^{\mathrm{b}}\end{array}$ & $\begin{array}{c}06 \\
(11.5)^{\mathrm{a}} \\
(17.1)^{\mathrm{b}}\end{array}$ & $\begin{array}{c}35 \\
(11.5)^{\mathrm{a}} \\
(100.0)^{\mathrm{b}}\end{array}$ \\
\hline $\mathrm{N}$ & $\begin{array}{c}143 \\
(47.3)^{\mathrm{a}} \\
(100.0)^{\mathrm{b}}\end{array}$ & $\begin{array}{c}70 \\
(23.2)^{\mathrm{a}} \\
(100.0)^{\mathrm{b}}\end{array}$ & $\begin{array}{c}35 \\
(11.5)^{\mathrm{a}} \\
(100.0)^{\mathrm{b}}\end{array}$ & $\begin{array}{c}52 \\
(17.2)^{\mathrm{a}} \\
(100.0)^{\mathrm{b}}\end{array}$ & $\begin{array}{c}302 \\
(100.0)^{\mathrm{a}} \\
(100.0)^{\mathrm{b}}\end{array}$ \\
\hline
\end{tabular}

Source: Same as Table 1

Note: Same as Table 1 
Table 22 reveals that more than two-thirds of sampled youth confirmed youth's involvement in violence, while $15.8 \%$ of them disagreed on this. The perceived reasons for youth involvement in violence included lack of jobs, unequal access to relief and developmental assistance, exclusion from education and employment, unequal access to social services, widespread corruption, restriction on cultural and social practices and economic security (see Table 23). Table 24 reveals that sampled youth expected reduction in youth violence after the abrogation of Article 370 through better employment opportunities (15.7\%), inclusive education (12.1\%), neutral and fair media (11.3\%), better governance and political institutions (10.5\%), economic security and reduced corruption $(10.1 \%)$ and skill development programs (8.1\%). Sampled youth were also aware of youth training schemes including skill development and entrepreneurship programs, but with considerably low utilization due to implementation apathy. However, about $40 \%$ of them expected better utilization of youth training schemes with robust implementation of these schemes after abrogation Article 370. About one-third of youth were aware of self-employment schemes and the level of awareness was reasonably high from Jammu district than other selected districts. More than half of sampled youth expected significant improvement in selfemployment schemes in near future.

Table 24. Ways to reduce youth violence after abrogation of Article 370 (No./\%)

\begin{tabular}{|c|c|c|c|c|c|}
\hline \multirow{2}{*}{ Ways } & \multicolumn{5}{|c|}{ Districts } \\
\hline & Jammu & Kathua & Rajouri & Poonch & $\mathrm{N}$ \\
\hline \multirow{3}{*}{$\begin{array}{l}\text { By providing employment } \\
\text { opportunities }\end{array}$} & 18 & 08 & 07 & 06 & 39 \\
\hline & $(20.6)^{\mathrm{a}}$ & $(8.7)^{\mathrm{a}}$ & $(22.6)^{\mathrm{a}}$ & $(15.7)^{\mathrm{a}}$ & $(15.7)^{\mathrm{a}}$ \\
\hline & $(46.2)^{b}$ & $(20.5)^{\mathrm{b}}$ & $(17.9)^{b}$ & $(15.4)^{\mathrm{b}}$ & $(100.0)^{\mathrm{b}}$ \\
\hline \multirow{3}{*}{$\begin{array}{l}\text { By providing skill development } \\
\text { programmes }\end{array}$} & 07 & 04 & 03 & 06 & 20 \\
\hline & $(8.04)^{\mathrm{a}}$ & $(4.3)^{\mathrm{a}}$ & $(9.6)^{\mathrm{a}}$ & $(15.7)^{\mathrm{a}}$ & $(8.1)^{\mathrm{a}}$ \\
\hline & $(35.0)^{\mathrm{b}}$ & $(20.0)^{\mathrm{b}}$ & $(15.0)^{\mathrm{b}}$ & $(30.0)^{b}$ & $(100.0)^{\mathrm{b}}$ \\
\hline \multirow[t]{3}{*}{ Neutral and fair media } & 11 & 07 & 06 & 04 & 28 \\
\hline & $(12.6)^{\mathrm{a}}$ & $(7.6)^{\mathrm{a}}$ & $(19.4)^{\mathrm{a}}$ & $(10.5)^{\mathrm{a}}$ & $(11.3)^{\mathrm{a}}$ \\
\hline & $(39.3)^{b}$ & $(25.0)^{\mathrm{b}}$ & $(21.4)^{\mathrm{b}}$ & $(14.2)^{b}$ & $(100.0)^{\mathrm{b}}$ \\
\hline \multirow{3}{*}{$\begin{array}{l}\text { Established well governed } \\
\text { political institution }\end{array}$} & 12 & 03 & 04 & 07 & 26 \\
\hline & $(13.7)^{\mathrm{a}}$ & $(3.3)^{\mathrm{a}}$ & $(12.9)^{\mathrm{a}}$ & $(18.4)^{\mathrm{a}}$ & $(10.5)^{\mathrm{a}}$ \\
\hline & $(46.2)^{b}$ & $(11.5)^{\mathrm{b}}$ & $(15.4)^{\mathrm{b}}$ & $(26.9)^{b}$ & $(100.0)^{\mathrm{b}}$ \\
\hline \multirow[t]{3}{*}{ Inclusion in education } & 14 & 06 & 05 & 05 & 30 \\
\hline & $(16.1)^{\mathrm{a}}$ & $(6.5)^{\mathrm{a}}$ & $(16.1)^{\mathrm{a}}$ & $(13.2)^{\mathrm{a}}$ & $(12.1)^{\mathrm{a}}$ \\
\hline & $(46.6)^{b}$ & $(20.0)^{\mathrm{b}}$ & $(16.6)^{b}$ & $(16.6)^{b}$ & $(100.0)^{b}$ \\
\hline \multirow[t]{3}{*}{ Economic security } & 11 & 06 & 02 & 06 & 25 \\
\hline & $(12.6)^{\mathrm{a}}$ & $(6.5)^{\mathrm{a}}$ & $(6.4)^{\mathrm{a}}$ & $(15.7)^{\mathrm{a}}$ & $(10.1)^{\mathrm{a}}$ \\
\hline & $(44.0)^{\mathrm{b}}$ & $(24.0)^{\mathrm{b}}$ & $(8.0)^{b}$ & $(24.0)^{\mathrm{b}}$ & $(100.0)^{\mathrm{b}}$ \\
\hline \multirow{3}{*}{$\begin{array}{l}\text { Need to address the issues of } \\
\text { corruption }\end{array}$} & 14 & 03 & 04 & 04 & 25 \\
\hline & $(16.1)^{\mathrm{a}}$ & $(3.3)^{\mathrm{a}}$ & $(12.9)^{\mathrm{a}}$ & $(10.5)^{\mathrm{a}}$ & $(10.1)^{\mathrm{a}}$ \\
\hline & $(56.0)^{\mathrm{b}}$ & $(12.0)^{\mathrm{b}}$ & $(16.0)^{b}$ & $(16.0)^{b}$ & $(100.0)^{\mathrm{b}}$ \\
\hline \multirow[t]{3}{*}{$\mathrm{N}$} & 87 & 91 & 31 & 38 & 247 \\
\hline & $(35.2)^{\mathrm{b}}$ & $(36.8)^{b}$ & $(12.5)^{\mathrm{b}}$ & $(15.3)^{\mathrm{b}}$ & $(100.0)^{b}$ \\
\hline & $(100.0)^{\mathrm{a}}$ & $(100.0)^{\mathrm{a}}$ & $(100.0)^{\mathrm{a}}$ & $(100.0)^{\mathrm{a}}$ & $(100.0)^{\mathrm{a}}$ \\
\hline
\end{tabular}

Source: Same as Table 1

Note: Same as Table 1

\subsection{Expectation of tourism development}

About $79.2 \%$ of sample youth reported a very high scope of tourism development in Jammu and Kashmir. Table 25 reveals that more than half of sampled youth expected flourishing tourism industry in coming years due to revocation of the Article 370 due to greater private investment in tourism infrastructure. Table 26 reveals that tourism generates more income $(17.8 \%)$, creates more jobs $(16.7 \%)$, provides more business for local people $(16.1 \%)$, improve the standard of 
living (13.8\%), attract more investment (13.1\%), diversify households (9.9\%), support museums and heritage sites (6.1\%) and generate foreign exchange earnings (6.1\%). Sampled youth expected high tourism development in near future with robust infrastructure development and compatible policies. The government is expected to provide the necessary infrastructure, transport facilities, tourism policies, financial investment, parking facilities, and restaurants at tourist places for the speedier development of the tourism industry.

Table 25. Expectations about flourishing of tourism industry (No./\%)

\begin{tabular}{|c|c|c|c|c|c|}
\hline \multirow{2}{*}{ Expectations } & \multicolumn{5}{|c|}{ Districts } \\
\hline & Jammu & Kathua & Rajouri & Poonch & $\mathrm{N}$ \\
\hline Same & $\begin{array}{c}05 \\
(10.2)^{\mathrm{a}} \\
(31.3)^{\mathrm{b}}\end{array}$ & $\begin{array}{c}06 \\
(22.2)^{\mathrm{a}} \\
(37.5)^{\mathrm{b}}\end{array}$ & $\begin{array}{c}03 \\
(15.0)^{\mathrm{a}} \\
(18.7)^{\mathrm{b}}\end{array}$ & $\begin{array}{c}02 \\
(8.3)^{\mathrm{a}} \\
(12.5)^{\mathrm{b}}\end{array}$ & $\begin{array}{c}16 \\
(13.3)^{\mathrm{a}} \\
(100.0)^{\mathrm{b}}\end{array}$ \\
\hline Improve & $\begin{array}{c}26 \\
(53.1)^{\mathrm{a}} \\
(39.4)^{\mathrm{b}}\end{array}$ & $\begin{array}{c}13 \\
(48.1)^{\mathrm{a}} \\
(19.6)^{\mathrm{b}}\end{array}$ & $\begin{array}{c}15 \\
(75.0)^{\mathrm{a}} \\
(22.7)^{\mathrm{b}}\end{array}$ & $\begin{array}{c}12 \\
(50.0)^{\mathrm{a}} \\
(18.2)^{\mathrm{b}}\end{array}$ & $\begin{array}{c}66 \\
(55.0)^{\mathrm{a}} \\
(100.0)^{\mathrm{b}}\end{array}$ \\
\hline Decline & $\begin{array}{c}02 \\
(4.1)^{\mathrm{a}} \\
(40.0)^{\mathrm{b}}\end{array}$ & $\begin{array}{c}01 \\
(3.7)^{\mathrm{a}} \\
(20.0)^{\mathrm{b}}\end{array}$ & - & $\begin{array}{c}02 \\
(8.3)^{\mathrm{a}} \\
(40.0)^{\mathrm{b}}\end{array}$ & $\begin{array}{c}05 \\
(4.2)^{\mathrm{a}} \\
(100.0)^{\mathrm{b}}\end{array}$ \\
\hline Cannot say & $\begin{array}{c}16 \\
(32.6)^{\mathrm{a}} \\
(48.5)^{\mathrm{b}}\end{array}$ & $\begin{array}{c}07 \\
(25.9)^{\mathrm{a}} \\
(21.2)^{\mathrm{b}}\end{array}$ & $\begin{array}{c}02 \\
(10.0)^{\mathrm{a}} \\
(6.1)^{\mathrm{b}}\end{array}$ & $\begin{array}{c}08 \\
(33.3)^{\mathrm{a}} \\
(24.2)^{\mathrm{b}}\end{array}$ & $\begin{array}{c}33 \\
(27.5)^{\mathrm{a}} \\
(100.0)^{\mathrm{b}}\end{array}$ \\
\hline $\mathrm{N}$ & $\begin{array}{c}49 \\
(40.8)^{\mathrm{b}} \\
(100.0)^{\mathrm{a}}\end{array}$ & $\begin{array}{c}27 \\
(22.5)^{\mathrm{b}} \\
(100.0)^{\mathrm{a}}\end{array}$ & $\begin{array}{c}20 \\
(16.6)^{\mathrm{b}} \\
(100.0)^{\mathrm{a}}\end{array}$ & $\begin{array}{c}24 \\
(20.0)^{\mathrm{b}} \\
(100.0)^{\mathrm{a}}\end{array}$ & $\begin{array}{c}120 \\
(100.0)^{\mathrm{b}} \\
(100.0)^{\mathrm{a}}\end{array}$ \\
\hline
\end{tabular}

Source: Same as Table 1

Note: Same as Table 1

Table 26. Contribution of tourism (No./\%)

\begin{tabular}{|c|c|c|c|c|c|}
\hline \multirow{2}{*}{ Contribution } & \multicolumn{5}{|c|}{ Districts } \\
\hline & Jammu & Kathua & Rajouri & Poonch & $\mathrm{N}$ \\
\hline Created more jobs & $\begin{array}{c}32 \\
(16.6)^{\mathrm{a}} \\
(40.5)^{\mathrm{b}}\end{array}$ & $\begin{array}{c}17 \\
(15.8)^{\mathrm{a}} \\
(21.5)^{\mathrm{b}}\end{array}$ & $\begin{array}{c}14 \\
(18.9)^{\mathrm{a}} \\
(17.7)^{\mathrm{b}}\end{array}$ & $\begin{array}{c}16 \\
(16.5)^{\mathrm{a}} \\
(20.2)^{\mathrm{b}}\end{array}$ & $\begin{array}{c}79 \\
(16.7)^{\mathrm{a}} \\
(100.0)^{\mathrm{b}}\end{array}$ \\
\hline Attract more investment & $\begin{array}{c}20 \\
(10.4)^{\mathrm{a}} \\
(32.2)^{\mathrm{b}}\end{array}$ & $\begin{array}{c}14 \\
(13.1)^{\mathrm{a}} \\
(22.5)^{\mathrm{b}}\end{array}$ & $\begin{array}{c}12 \\
(16.2)^{\mathrm{a}} \\
(19.3)^{\mathrm{b}}\end{array}$ & $\begin{array}{c}16 \\
(16.5)^{\mathrm{a}} \\
(25.8)^{\mathrm{b}}\end{array}$ & $\begin{array}{c}62 \\
(13.1)^{\mathrm{a}} \\
(100.0)^{\mathrm{b}}\end{array}$ \\
\hline Provide more business for local people & $\begin{array}{c}33 \\
(17.1)^{\mathrm{a}} \\
(43.4)^{\mathrm{b}}\end{array}$ & $\begin{array}{c}18 \\
(16.8)^{\mathrm{a}} \\
(23.6)^{\mathrm{b}}\end{array}$ & $\begin{array}{c}07 \\
(9.4)^{\mathrm{a}} \\
(9.2)^{\mathrm{b}}\end{array}$ & $\begin{array}{c}18 \\
(18.5)^{\mathrm{a}} \\
(23.6)^{\mathrm{b}}\end{array}$ & $\begin{array}{c}76 \\
(16.1)^{\mathrm{a}} \\
(100.0)^{\mathrm{b}}\end{array}$ \\
\hline Support museums and heritage site & $\begin{array}{c}12 \\
(6.2)^{\mathrm{a}} \\
(41.4)^{\mathrm{b}}\end{array}$ & $\begin{array}{c}06 \\
(5.6)^{\mathrm{a}} \\
(20.6)^{\mathrm{b}}\end{array}$ & $\begin{array}{c}06 \\
(8.1)^{\mathrm{a}} \\
(20.6)^{\mathrm{b}}\end{array}$ & $\begin{array}{c}05 \\
(5.1)^{\mathrm{a}} \\
(17.2)^{\mathrm{b}}\end{array}$ & $\begin{array}{c}29 \\
(6.1)^{\mathrm{a}} \\
(100.0)^{\mathrm{b}}\end{array}$ \\
\hline $\begin{array}{l}\text { Diversified households and community } \\
\text { livelihood choices and opportunities }\end{array}$ & $\begin{array}{c}19 \\
(9.8)^{\mathrm{a}} \\
(40.4)^{\mathrm{b}}\end{array}$ & $\begin{array}{c}13 \\
(12.1)^{\mathrm{a}} \\
(27.6)^{\mathrm{b}}\end{array}$ & $\begin{array}{c}07 \\
(9.4)^{\mathrm{a}} \\
(14.8)^{\mathrm{b}}\end{array}$ & $\begin{array}{c}08 \\
(8.2)^{\mathrm{a}} \\
(17.0)^{\mathrm{b}}\end{array}$ & $\begin{array}{c}47 \\
(9.9)^{\mathrm{a}} \\
(100.0)^{\mathrm{b}}\end{array}$ \\
\hline Generate more income & $\begin{array}{c}33 \\
(17.1)^{\mathrm{a}} \\
(39.3)^{\mathrm{b}}\end{array}$ & $\begin{array}{c}19 \\
(17.7)^{\mathrm{a}} \\
(22.6)^{\mathrm{b}}\end{array}$ & $\begin{array}{c}16 \\
(21.6)^{\mathrm{a}} \\
(19.0)^{\mathrm{b}}\end{array}$ & $\begin{array}{c}16 \\
(16.5)^{\mathrm{a}} \\
(19.0)^{\mathrm{b}}\end{array}$ & $\begin{array}{c}84 \\
(17.8)^{\mathrm{a}} \\
(100.0)^{\mathrm{b}}\end{array}$ \\
\hline Improvement in the standard of living & $\begin{array}{c}30 \\
(15.5)^{\mathrm{a}} \\
(46.2)^{\mathrm{b}}\end{array}$ & $\begin{array}{c}14 \\
(13.1)^{\mathrm{a}} \\
(21.5)^{\mathrm{b}}\end{array}$ & $\begin{array}{c}08 \\
(10.8)^{\mathrm{a}} \\
(12.3)^{\mathrm{b}}\end{array}$ & $\begin{array}{c}13 \\
(13.4)^{\mathrm{a}} \\
(20.0)^{\mathrm{b}}\end{array}$ & $\begin{array}{c}65 \\
(13.8)^{\mathrm{a}} \\
(100.0)^{\mathrm{b}}\end{array}$ \\
\hline Foreign exchange earnings & $\begin{array}{c}14 \\
(7.2)^{\mathrm{a}} \\
(48.3)^{\mathrm{b}}\end{array}$ & $\begin{array}{c}06 \\
(5.6)^{\mathrm{a}} \\
(20.6)^{\mathrm{b}}\end{array}$ & $\begin{array}{c}04 \\
(5.4)^{\mathrm{a}} \\
(13.7)^{\mathrm{b}}\end{array}$ & $\begin{array}{c}05 \\
(5.1)^{\mathrm{a}} \\
(17.2)^{\mathrm{b}}\end{array}$ & $\begin{array}{c}29 \\
(6.1)^{\mathrm{a}} \\
(100.0)^{\mathrm{b}}\end{array}$ \\
\hline $\mathrm{N}$ & $\begin{array}{c}193 \\
(40.9)^{\mathrm{b}} \\
(100.0)^{\mathrm{a}}\end{array}$ & $\begin{array}{c}107 \\
(22.7)^{\mathrm{b}} \\
(100.0)^{\mathrm{a}}\end{array}$ & $\begin{array}{c}74 \\
(15.7)^{\mathrm{b}} \\
(100.0)^{\mathrm{a}}\end{array}$ & $\begin{array}{c}97 \\
(20.5)^{\mathrm{b}} \\
(100.0)^{\mathrm{a}}\end{array}$ & $\begin{array}{c}471 \\
(100.0)^{\mathrm{b}} \\
(100.0)^{\mathrm{a}}\end{array}$ \\
\hline
\end{tabular}

Source: Same as Table 1

Note: Same as Table 1 


\subsection{Expectation of horticulture and handicrafts development}

Table 27. Contribution of horticulture sector in the economy (No./\%)

\begin{tabular}{|c|c|c|c|c|c|}
\hline \multirow{2}{*}{ Contribution } & \multicolumn{5}{|c|}{ Districts } \\
\hline & Jammu & Kathua & Rajouri & Poonch & $\mathrm{N}$ \\
\hline \multirow[t]{3}{*}{ Raise income level } & 20 & 11 & 11 & 12 & 54 \\
\hline & $(13.9)^{\mathrm{a}}$ & $(15.1)^{\mathrm{a}}$ & $(19.2)^{\mathrm{a}}$ & $(19.0)^{\mathrm{a}}$ & $(16.1)^{\mathrm{a}}$ \\
\hline & $(37.0)^{\mathrm{b}}$ & $(20.3)^{\mathrm{b}}$ & $(20.3)^{b}$ & $(22.2)^{\mathrm{b}}$ & $(100.0)^{\mathrm{b}}$ \\
\hline \multirow[t]{3}{*}{ Generate employment } & 29 & 14 & 08 & 10 & 61 \\
\hline & $(20.3)^{\mathrm{a}}$ & $(19.2)^{\mathrm{a}}$ & $(14.0)^{\mathrm{a}}$ & $(15.8)^{\mathrm{a}}$ & $(18.2)^{\mathrm{a}}$ \\
\hline & $(47.5)^{\mathrm{b}}$ & $(22.9)^{\mathrm{b}}$ & $(13.1)^{\mathrm{b}}$ & $(16.4)^{b}$ & $(100.0)^{b}$ \\
\hline \multirow[t]{3}{*}{ Alleviate poverty } & 20 & 10 & 06 & 08 & 44 \\
\hline & $(13.9)^{\mathrm{a}}$ & $(13.6)^{\mathrm{a}}$ & $(10.5)^{\mathrm{a}}$ & $(12.6)^{\mathrm{a}}$ & $(13.1)^{\mathrm{a}}$ \\
\hline & $(45.4)^{\mathrm{b}}$ & $(22.7)^{b}$ & $(13.6)^{b}$ & $(18.2)^{b}$ & $(100.0)^{\mathrm{b}}$ \\
\hline \multirow[t]{3}{*}{ Export promotion } & 20 & 09 & 13 & 11 & 53 \\
\hline & $(13.9)^{\mathrm{a}}$ & $(12.3)^{\mathrm{a}}$ & $(22.8)^{\mathrm{a}}$ & $(17.5)^{\mathrm{a}}$ & $(15.7)^{\mathrm{a}}$ \\
\hline & $(37.7)^{b}$ & $(16.9)^{\mathrm{b}}$ & $(24.5)^{\mathrm{b}}$ & $(20.7)^{b}$ & $(100.0)^{\mathrm{b}}$ \\
\hline \multirow{3}{*}{ Provides food and nutritional secure } & 23 & 11 & 09 & 10 & 53 \\
\hline & $(16.1)^{\mathrm{a}}$ & $(15.1)^{\mathrm{a}}$ & $(15.7)^{\mathrm{a}}$ & $(15.8)^{\mathrm{a}}$ & $(15.7)^{\mathrm{a}}$ \\
\hline & $(43.4)^{\mathrm{b}}$ & $(20.7)^{\mathrm{b}}$ & $(16.9)^{\mathrm{b}}$ & $(18.8)^{\mathrm{b}}$ & $(100.0)^{\mathrm{b}}$ \\
\hline \multirow[t]{3}{*}{ Maintain ecological balance } & 17 & 09 & 05 & 06 & 37 \\
\hline & $(11.8)^{\mathrm{a}}$ & $(12.3)^{\mathrm{a}}$ & $(8.7)^{\mathrm{a}}$ & $(9.5)^{\mathrm{a}}$ & $(11.0)^{\mathrm{a}}$ \\
\hline & $(45.9)^{\mathrm{b}}$ & $(24.3)^{\mathrm{b}}$ & $(13.5)^{b}$ & $(16.2)^{\mathrm{b}}$ & $(100.0)^{\mathrm{b}}$ \\
\hline \multirow[t]{3}{*}{ Provides raw materials } & 14 & 09 & 05 & 06 & 34 \\
\hline & $(9.7)^{\mathrm{a}}$ & $(12.3)^{\mathrm{a}}$ & $(8.7)^{\mathrm{a}}$ & $(9.5)^{\mathrm{a}}$ & $(10.1)^{\mathrm{a}}$ \\
\hline & $(41.2)^{\mathrm{b}}$ & $(26.5)^{\mathrm{b}}$ & $(14.7)^{b}$ & $(17.6)^{\mathrm{b}}$ & $(100.0)^{\mathrm{b}}$ \\
\hline \multirow[t]{3}{*}{$\mathrm{N}$} & 143 & 73 & 57 & 63 & 336 \\
\hline & $(42.5)^{b}$ & $(21.7)^{\mathrm{b}}$ & $(16.9)^{\mathrm{b}}$ & $(18.7)^{\mathrm{b}}$ & $(100.0)^{\mathrm{b}}$ \\
\hline & $(100.0)^{\mathrm{a}}$ & $(100.0)^{\mathrm{a}}$ & $(100.0)^{\mathrm{a}}$ & $(100.0)^{\mathrm{a}}$ & $(100.0)^{\mathrm{a}}$ \\
\hline
\end{tabular}

Source: Same as Table 1

Note: Same as Table 1

Table 28. Ways to boost horticulture sector (No./\%)

\begin{tabular}{|c|c|c|c|c|c|}
\hline \multirow{2}{*}{ Ways } & \multicolumn{5}{|c|}{ Districts } \\
\hline & Jammu & Kathua & Rajouri & Poonch & $\mathrm{N}$ \\
\hline Transportation & $\begin{array}{c}26 \\
(19.8)^{\mathrm{a}} \\
(41.9)^{\mathrm{b}}\end{array}$ & $\begin{array}{c}16 \\
(22.8)^{\mathrm{a}} \\
(25.8)^{\mathrm{b}}\end{array}$ & $\begin{array}{c}09 \\
(15.3)^{\mathrm{a}} \\
(14.5)^{\mathrm{b}}\end{array}$ & $\begin{array}{c}11 \\
(15.4)^{\mathrm{a}} \\
(17.7)^{\mathrm{b}}\end{array}$ & $\begin{array}{c}62 \\
(18.7)^{\mathrm{a}} \\
(100.0)^{\mathrm{b}}\end{array}$ \\
\hline Storage facilities & $\begin{array}{c}29 \\
(22.1)^{\mathrm{a}} \\
(43.3)^{\mathrm{b}}\end{array}$ & $\begin{array}{c}14 \\
(20.0)^{\mathrm{a}} \\
(20.8)^{\mathrm{b}}\end{array}$ & $\begin{array}{c}11 \\
(18.6)^{\mathrm{a}} \\
(16.4)^{\mathrm{b}}\end{array}$ & $\begin{array}{c}13 \\
(18.3)^{\mathrm{a}} \\
(19.4)^{\mathrm{b}}\end{array}$ & $\begin{array}{c}67 \\
(20.2)^{\mathrm{a}} \\
(100.0)^{\mathrm{b}}\end{array}$ \\
\hline Skilled labour & $\begin{array}{c}27 \\
(20.6)^{\mathrm{a}} \\
(38.0)^{\mathrm{b}}\end{array}$ & $\begin{array}{c}12 \\
(17.1)^{\mathrm{a}} \\
(16.9)^{\mathrm{b}}\end{array}$ & $\begin{array}{c}12 \\
(20.3)^{\mathrm{a}} \\
(16.9)^{\mathrm{b}}\end{array}$ & $\begin{array}{c}20 \\
(28.2)^{\mathrm{a}} \\
(28.2)^{\mathrm{b}}\end{array}$ & $\begin{array}{c}71 \\
(21.4)^{\mathrm{a}} \\
(100.0)^{\mathrm{b}}\end{array}$ \\
\hline Market strategy & $\begin{array}{c}28 \\
(21.4)^{\mathrm{a}} \\
(37.3)^{\mathrm{b}}\end{array}$ & $\begin{array}{c}16 \\
(22.8)^{\mathrm{a}} \\
(21.3)^{\mathrm{b}}\end{array}$ & $\begin{array}{c}16 \\
(27.1)^{\mathrm{a}} \\
(21.3)^{\mathrm{b}}\end{array}$ & $\begin{array}{c}15 \\
(21.1)^{\mathrm{a}} \\
(20.0)^{\mathrm{b}}\end{array}$ & $\begin{array}{c}75 \\
(22.6)^{\mathrm{a}} \\
(100.0)^{\mathrm{b}}\end{array}$ \\
\hline Finance & $\begin{array}{c}21 \\
(16.0)^{\mathrm{a}} \\
(28.0)^{\mathrm{b}}\end{array}$ & $\begin{array}{c}12 \\
(17.1)^{\mathrm{a}} \\
(16.0)^{\mathrm{b}}\end{array}$ & $\begin{array}{c}11 \\
(18.6)^{\mathrm{a}} \\
(14.6)^{\mathrm{b}}\end{array}$ & $\begin{array}{c}12 \\
(16.9)^{\mathrm{a}} \\
(16.0)^{\mathrm{b}}\end{array}$ & $\begin{array}{c}56 \\
(16.9)^{\mathrm{a}} \\
(100.0)^{\mathrm{b}}\end{array}$ \\
\hline $\mathrm{N}$ & $\begin{array}{c}131 \\
(39.5)^{\mathrm{b}} \\
(100.0)^{\mathrm{a}}\end{array}$ & $\begin{array}{c}70 \\
(21.1)^{\mathrm{b}} \\
(100.0)^{\mathrm{a}}\end{array}$ & $\begin{array}{c}59 \\
(17.8)^{\mathrm{b}} \\
(100.0)^{\mathrm{a}}\end{array}$ & $\begin{array}{c}71 \\
(21.4)^{\mathrm{b}} \\
(100.0)^{\mathrm{a}}\end{array}$ & $\begin{array}{c}331 \\
(100.0)^{\mathrm{b}} \\
(100.0)^{\mathrm{a}}\end{array}$ \\
\hline
\end{tabular}

Source: Same as Table 1

Note: Same as Table 1 
Horticulture sector plays an important role in the development of Jammu and Kashmir. Horticulture sector generates significant employment, increases income level, promotes exports, alleviates poverty and maintains ecological balance (see Table 27). Sampled youth argued that government should improve transportation facilities and storage facilities including supply of skilled labor and finance for horticulture development (see Table 28). More than half of sampled youth rate expected increase in the contribution of handicrafts sector in near future for which the problems of lack of skilled workers, low availability of capital, poor infrastructure, and absence of marketing intelligence should be addressed on priority (see Table 29). Besides, product marketing, financial assistance, interest free loan, and handicrafts clusters should be provided.

Table 29. Contribution of handicrafts to economy (No./\%)

\begin{tabular}{cccccc}
\hline \multirow{2}{*}{ Contribution } & \multicolumn{5}{c}{ Districts } \\
\cline { 2 - 6 } & Jammu & Kathua & Rajouri & Poonch & $\mathrm{N}$ \\
\hline Low & 14 & 05 & 06 & 04 & 29 \\
& $(35.0)^{\mathrm{a}}$ & $(20.8)^{\mathrm{a}}$ & $(46.2)^{\mathrm{a}}$ & $(25.0)^{\mathrm{a}}$ & $(31.2)^{\mathrm{a}}$ \\
& $(48.3)^{\mathrm{b}}$ & $(17.2)^{\mathrm{b}}$ & $(20.6)^{\mathrm{b}}$ & $(13.7)^{\mathrm{b}}$ & $(100.0)^{\mathrm{b}}$ \\
Moderate & 21 & 15 & 06 & 09 & 51 \\
& $(52.5)^{\mathrm{a}}$ & $(62.5)^{\mathrm{a}}$ & $(46.2) \mathrm{a}$ & $(56.2)^{\mathrm{a}}$ & $(54.8)^{\mathrm{a}}$ \\
& $(41.2)^{\mathrm{b}}$ & $(29.4)^{\mathrm{b}}$ & $(11.7)^{\mathrm{b}}$ & $(17.6)^{\mathrm{b}}$ & $(100.0)^{\mathrm{b}}$ \\
High & 05 & 04 & 01 & 03 & 13 \\
& $(12.5)^{\mathrm{a}}$ & $(16.6)^{\mathrm{a}}$ & $(7.6)^{\mathrm{a}}$ & $(18.7)^{\mathrm{a}}$ & $(13.9)^{\mathrm{a}}$ \\
& $(38.4)^{\mathrm{b}}$ & $(30.7)^{\mathrm{b}}$ & $(7.6)^{\mathrm{b}}$ & $(23.1)^{\mathrm{b}}$ & $(100.0)^{\mathrm{b}}$ \\
& 40 & 24 & 13 & 16 & 93 \\
& $(43.0)^{\mathrm{b}}$ & $(25.8)^{\mathrm{b}}$ & $(13.9)^{\mathrm{b}}$ & $(17.2)^{\mathrm{b}}$ & $(100.0)^{\mathrm{b}}$ \\
& $(100.0)^{\mathrm{a}}$ & $(100.0)^{\mathrm{a}}$ & $(100.0)^{\mathrm{a}}$ & $(100.0)^{\mathrm{a}}$ & $(100.0)^{\mathrm{a}}$ \\
\hline
\end{tabular}

Source: Same as Table 1

Note: Same as Table 1

Table 30. Ways to improve the performance of handicrafts sector (No./\%)

\begin{tabular}{|c|c|c|c|c|c|}
\hline \multirow{2}{*}{ Ways } & \multicolumn{5}{|c|}{ Districts } \\
\hline & Jammu & Kathua & Rajouri & Poonch & $\mathrm{N}$ \\
\hline Product marketing & $\begin{array}{c}21 \\
(16.2)^{\mathrm{a}} \\
(40.4)^{\mathrm{b}}\end{array}$ & $\begin{array}{c}15 \\
(21.1)^{\mathrm{a}} \\
(28.8)^{\mathrm{b}}\end{array}$ & $\begin{array}{c}11 \\
(21.5)^{\mathrm{a}} \\
(21.2)^{\mathrm{b}}\end{array}$ & $\begin{array}{c}05 \\
(9.6)^{\mathrm{a}} \\
(9.6)^{\mathrm{b}}\end{array}$ & $\begin{array}{c}52 \\
(17.1)^{\mathrm{a}} \\
(100.0)^{\mathrm{b}}\end{array}$ \\
\hline Financial assistance & $\begin{array}{c}23 \\
(17.6)^{\mathrm{a}} \\
(37.7)^{\mathrm{b}}\end{array}$ & $\begin{array}{c}12 \\
(16.9)^{\mathrm{a}} \\
(19.6)^{\mathrm{b}}\end{array}$ & $\begin{array}{c}08 \\
(15.6)^{\mathrm{a}} \\
(13.1)^{\mathrm{b}}\end{array}$ & $\begin{array}{c}10 \\
(19.2)^{\mathrm{a}} \\
(16.3)^{\mathrm{b}}\end{array}$ & $\begin{array}{c}61 \\
(20.1)^{\mathrm{a}} \\
(100.0)^{\mathrm{b}}\end{array}$ \\
\hline Interest free loan & $\begin{array}{c}21 \\
(16.2)^{\mathrm{a}} \\
(43.7)^{\mathrm{b}}\end{array}$ & $\begin{array}{c}09 \\
(12.6)^{\mathrm{a}} \\
(18.7)^{\mathrm{b}}\end{array}$ & $\begin{array}{c}08 \\
(15.6)^{\mathrm{a}} \\
(16.6)^{\mathrm{b}}\end{array}$ & $\begin{array}{c}10 \\
(19.2)^{\mathrm{a}} \\
(20.8)^{\mathrm{b}}\end{array}$ & $\begin{array}{c}48 \\
(15.7)^{\mathrm{a}} \\
(100.0)^{\mathrm{b}}\end{array}$ \\
\hline Establish clusters of Handicrafts & $\begin{array}{c}33 \\
(25.4)^{\mathrm{a}} \\
(42.8)^{\mathrm{b}}\end{array}$ & $\begin{array}{c}17 \\
(23.9)^{\mathrm{a}} \\
(22.1)^{\mathrm{b}}\end{array}$ & $\begin{array}{c}14 \\
(27.4)^{\mathrm{a}} \\
(18.2)^{\mathrm{b}}\end{array}$ & $\begin{array}{c}13 \\
(25.0)^{\mathrm{a}} \\
(16.8)^{\mathrm{b}}\end{array}$ & $\begin{array}{c}77 \\
(25.3)^{\mathrm{a}} \\
(100.0)^{\mathrm{b}}\end{array}$ \\
\hline Skilled labour & $\begin{array}{c}32 \\
(24.6)^{\mathrm{a}} \\
(43.2)^{\mathrm{b}}\end{array}$ & $\begin{array}{c}18 \\
(25.3)^{\mathrm{a}} \\
(24.3)^{\mathrm{b}}\end{array}$ & $\begin{array}{c}10 \\
(19.6)^{\mathrm{a}} \\
(13.5)^{\mathrm{b}}\end{array}$ & $\begin{array}{c}14 \\
(26.9)^{\mathrm{a}} \\
(18.9)^{\mathrm{b}}\end{array}$ & $\begin{array}{c}74 \\
(24.3)^{\mathrm{a}} \\
(100.0)^{\mathrm{b}}\end{array}$ \\
\hline $\mathrm{N}$ & $\begin{array}{c}130 \\
(42.7)^{\mathrm{b}} \\
(100.0)^{\mathrm{a}}\end{array}$ & $\begin{array}{c}71 \\
(23.3)^{\mathrm{b}} \\
(100.0)^{\mathrm{a}}\end{array}$ & $\begin{array}{c}51 \\
(16.7)^{\mathrm{b}} \\
(100.0)^{\mathrm{a}}\end{array}$ & $\begin{array}{c}52 \\
(17.1)^{\mathrm{b}} \\
(100.0)^{\mathrm{a}}\end{array}$ & $\begin{array}{c}304 \\
(100.0)^{\mathrm{b}} \\
(100.0)^{\mathrm{a}}\end{array}$ \\
\hline
\end{tabular}

Source: Same as Table 1

Note: Same as Table 1 


\subsection{Expectation of economic recovery}

There are different ways to start economic recovery, which include revenue mobilization, trade, investment, local participation, reduction in corruption, transparency, infrastructure development, agriculture reforms, and development of handicrafts and horticulture. The role of the Central government to start economic recovery is emphasized by large proportion of sampled youth. Besides, proper policy and planning, policy reforms, economic management, public expenditure and private investment are essential for speedier economic recovery. Health, education, trade, banking, agriculture, industry, transport, power, handicrafts, tourism and renewable energy should be adequately focused for economic recovery. It has been emphasized that people participation in development of Jammu and Kashmir is sine qua non for economic recovery after abrogation of the Article 370 . However, participatory economic recovery is not possible without addressing the challenges of inadequate public awareness, lack of social capital and trust of local people. Investment has been considered as an important determining factor in economic recovery. The revocation of Article 370 will allow the private sector to invest in different sectors and opens up the opportunities in pharmaceuticals, leather, education, health and information technology. Significant investment is needed for development of tourism, businesses, educational institutions, hospitals, roads, railways, industry and agriculture for overall prosperity and growth.

\section{Conclusion and policy implications}

From the decades, the economy of Jammu and Kashmir was considered as backward as it lacks economic and social development. This becomes one of the reasons for the revocation of special status of Jammu and Kashmir. The chief characteristics of economic backwardness of Jammu and Kashmir include the predominance of the agriculture sector, high birthrate, debilitated infrastructure, scattered population, low degree of urbanization, widespread illiteracy, corruption, abysmal mass poverty, institutional decay, Inequality, exclusion, low level of investment, low-income generation, natural disasters, poor connectivity, mountainous and remote area, inhospitable terrain, poor policy design, militancy, and economic mismanagement. Article 370 was abrogated by the Government of India on the fifth of August 2019 in light of fact that this was considered as the primary reason behind the economic backwardness Jammu and Kashmir. Article 370 restricted the economy of Jammu and Kashmir to grow compared to other Indian states. For instance, Jammu and Kashmir failed to attract much needed private and foreign investment specifically in education, health, horticulture, handicrafts and tourism. Economic reforms have not been initiated and even extended to Jammu and Kashmir. The economy was highly gripped by widespread corruption, poverty, poor accountability, prevailinghorizontal inequalities. Agriculture and industrial sectors remained underdeveloped. Youth violence, unemployment, and terrorism were pervasive. The development programs of the Government of India were not properly implemented. All these factors denied Jammu and Kashmir the benefits of robust economic growth in India in recent years. Therefore, the Government of India abrogated Article 370 in order to boost up the economy of Jammu and Kashmir by allowing the private investment in education, health, and tourism with the expectation that it will usher new opportunities of economic growth in Jammu and Kashmir, which may generate much needed employment and ameliorate the livelihoods of the masses.

The study reveals that youth participation in economic recovery is vital to give right direction to the economy specifically since the revocation of special status of Jammu and Kashmir. The developmental aspirations of the youth focused on agriculture reforms, investment in manufacturing, transportation, health, education, infrastructure, banking, and transparency in all development planning and implementation. Youth education and training and self-employment including the development of tourism, horticulture and handicrafts sectors were prioritized for economic recovery during the post-abrogation of Article 370. Addressing persistently high unemployment rate in Jammu and Kashmir has been ranked as one of the top priority area for economic recovery. There is need to rejuvenate the sick industrial units for creating sustained employment opportunities for youth. Tourism, handicrafts and horticulture sectors should be developed to reduce poverty, promote exports and generate employment for the youth through substantial funding from the Central government and private sector.

Investment in the manufacturing sector should focus on the promotion and development of agro-processing, pharmaceuticals, industry, textiles, films, motorcycles, and electronics, besides other small scale enterprises. The availability, accessibility, affordability and quality of health services should be improved substantially to provide high- 
quality preventive and corrective measures. The existing education sector should be strengthened to provide quality education to the youth and address the gaps between curriculum and industry demand to unlock multiple employment opportunities. Holistic growth and expansion of handicrafts and horticulture should be achieved. Handicrafts clusters should be developed throughout the region. Horticultural infrastructure such as proper market facilities, transportation, storage facilities, and skilled labor should be promoted. There is a need to involve the local community including youth in development planning and decision making for ensuring accountability and transparency in economic recovery of Jammu and Kashmir.

\section{References}

Abrol, V. (2014). Jammu \& Kashmir Bank : A case study. Electronic International Interdisciplinary Research Journal, 3(3), 76-101.

Amsami, A. (2019). Post-conflict peace-building and economic recovery in North Eastern Nigeria. International Journal of Scientific \& Engineering Research, 10(10), 333-347.

Appadurai, A. (2004). The capacity to aspire: Culture and the terms of recognition. In V. Rao \& M. Walton (Eds.), Culture and Public Action. Stanford, CA: Stanford University Press.

Bandura, A. (1977). Social Learning Theory. Englewood Cliffs, NJ: Prentice-Hall.

Bhat, B. A. (2019). A study on Jammu and Kashmir present, past and views of students on Article 370 Abrogation. International Journal of Latest Research in Humanities and Social Science, 2(12), 1-11.

Bhat, I. A. (2017). Economic potential of handicrafts of Jammu and Kashmir. International Journal of Science and Research, 6(12), 496-501.

Bhat, J., \& Yadav, P. (2016). Handicraft sector: The comforting sector of employment review. Management Studies and Economic Systems, 3(2), 111-117.

Bhattacharyya, S., \& Jha, R. (2013). Economic growth, law and corruption: Evidence from India. Comparative Economic Studies, 55(2), 287-313.

Bhosale (2014). Role of Indian banking sector in the economic growth and development. International General of Management Research, 2(12), 208-219.

Breuer, A., Frumusanu, N. M., \& Bilianciurea, J. (2010). Investments and economic recovery. Directory of open access Journals, 10(1), 29-36.

Chang, K.-S., Ben, F., \& Linda, W. (2012). Developmental Politics in Transition: The Neoliberal Era and Beyond. Switzerland: Springer Nature.

Chang, S. E., \& Rose, A. Z. (2012). Towards a theory of economic recovery from disasters. Clinical Research Educational Artistic And Therapeutic Endeavors, 32(2), 171-181.

Dalton, P., Ghosal, S., \& Mani, A. (2016). Poverty and aspirations failure. The Economic Journal, 126(590), $165-188$.

Darzi, M. I. (2016). Horticulture sector towards economic development of Jammu \& Kashmir. International Journal of Multidisciplinary Research and Development, 6(4), 238-240.

Dimant, E., \& Tosato, G. (2018). Causes and effects of economic corruption:What has past decade's empirical research teach us? A survey. Journal of Economic Surveys, 32(2), 335-356.

Drèze, J., \& Sen, A. (2002). India: Development and Participation. Oxford: Oxford University Press.

Erden, L., \& Holcombe, R. G. (2006). The linkage between the public and private investment A co-integration analysis of a panel of developing countries. Eastern Economic Journal, 32(3), 479-492.

Gachunga, M. J., \& Kuso, Y. (2019). Contribution of infrastructure to economic growth in Africa. International Journal of Business Marketing and Management, 4(4), 43-51.

ILO (2014). Transforming economies: Making industrial policy work for growth, jobs and development. Geneva: International Labour Organization.

ILO (2010). Local economic recovery in post-conflict: Guidelines. Geneva: International Labour Organization.

Islam, R. T., \& Shrivastava, S. (2017). A study on area, production and productivity of apples in J \& K from 2006-07 to 2015-16. International Journal of Scientific Research and Management, 5(7), 6513-6519.

Jomo, K. S., \& Chowdhury, A. (2020). COVID-19 pandemic: Recession and recovery. Development, 63, $226-237$.

Kedia, S., Pandey, R., \& Sinha R. (2020). Shaping the post-COVID-19 development paradigm in India: Some imperatives for greening the economic recovery. Millennial Asia, 11(3), 268-298.

Keen, D. (2003). Greedy elites, dwindling resources, alienated youths: The anatomy of protracted violence in Sierra Leone. International Politik and Gesellschaft, 3(2), 67-94. 
Khan, B. A. (2013). Unemployment and employment pattern in Jammu and Kashmir: A case study of Kupwara district. International Journal of Educational Research and Technology, 4(1), 79-89.

Khatik, D., \& Nag, A. (2012). Role of tourism industry in economic development of India. International Journal of Management and Business Affairs, 2(3), 44-51.

Liu, J., Tang, J., Zhou, B., \& Liang, Z. (2018). The effect of governance quality on economic growth: Based on China's provincial panel data. Economies, 6, 56.

Lone, R. A. (2014). Horticulture sector in Jammu and Kashmir economy. European Academic Research, 2(2), 24052431.

Lybbert, T. J., \& Wydick, B. (2018). Poverty, aspirations, and the economics of hope. Economic Development and Cultural Change, 66(4), 709-753.

Mahapatra, D. A., \& Shekhawat, S. (2008). The peace process and prospects for economic reconstruction in Kashmir. Peace and Conflict Review, 3(1), 1-17.

Mills, F. C. (1934). Economic recovery. American Journal of Sociology, 39(6), 738-748.

Mir, H. A. (2014). Impact of tourism industry on economic development of Jammu and Kashmir. International Journal of Scientific \& Engineering Research, 5(6), 592-598.

Mir, H. A., \& Bhat, S. A. (2018). Health status and access to health care services in Jammu and Kashmir state. Asian Review of Social Sciences, 7(3), 52-57.

Mir, R. A. (2019). The institutionalization of corruption and its impact on political culture in Jammu and Kashmir. International Journal of Social Science and Economic Research, 4(1), 447-456.

Mohanty, M. S. (1997). Macro-economic stability, growth and fiscal reform: The Indian perspective. Economic and Political Weekly, 32(6), 289-298.

Moore, K. (2019). The quest for sustainable employment: Challenges faced by young people during the job-search process. Australian Journal of Social Issues, 54, 91-108.

Nayyar, G. (2008). Economic growth and regional inequality in India. Economic and Political Weekly, 43(6), 58-67.

Nengroo, A. H. (2015). Employment and unemployment scenario of Jammu and Kashmir. International Journal of Scientific Research in Science and Technology, 1(3), 133-139.

Perkins, J. O. (1979). Aspects of macroeconomic policy. The Australian Economic Review, 12(1), 90-94.

Qadri, B., \& Kasab, M. A. (2017). Educational unemployment in Jammu and Kashmir: Causes, consequences and remedial measures. Asian Journal of Managerial Science, 6(2), 58-66.

Ray, D. (2006). Aspirations, poverty and economic change. In A. V. Banerjee, R. Bénabou \& D. Mookherjee (Eds.), Understanding Poverty. Oxford: Oxford University Press.

Rehman, O. U. (2016). Indian banking sector impact on economic growth rate and social development of rural areas of India. International Journal of Management and Commerce Innovations, 3(2), 612-623.

Robeyns, I. (2016). Capabilitarianism. Journal of Human Development and Capabilities, 17(3), 397-414.

Rose, A., \& Krausmann, E. (2013). An economic framework for the development of a resilience index for business recovery. International Journal of Disaster Risk Reduction, 5, 73-83.

Sen, A. (1985). Well-being, agency and freedom: The dewey lectures 1984. The Journal of Philosophy, 82(4), 169-221.

Shah, H. (2018). School education in Jammu and Kashmir: Issues and concerns. An International Jiurnal of Education and Applied Social Science, 9(1), 107-113.

Singh, S. (2017). Economic features of Jammu and Kashmir economy. The International Journal of Engineering Development and Research, 5(4), 578-581.

Snyder, C. R. (2002). Hope theory: Rainbows in the mind. Psychological Inquiry, 13(4), 249-275.

Sreenivasulu, S. E. (2013). Role and importance of educational for effective growth of Indian economy: An overview. Journal of Humanities and Social Science, 7(5), 32-35.

Strohmaier, R., Schuetz, M., \& Vannuccini, S. (2019). A systemic perspective on socioeconomic transformation in the digital age. Journal of Industrial and Business Economics, 46, 361-378.

Summers, L. H. (2010). Principles for economic recovery and renewal. Business Economics, 45(1), 3-7.

Thanoon, M. A., Baharumshah, A. Z., \& Rahman, A. A. (2014). Malaysia from economic recovery to sustained economic growth. Journal of Post Keynesian Economics, 28(2), 295-315.

Upadhyay, R. K. (2019). Opportunities for growth and investment in Jammu and Kashmir. AAYAM Journal of Management, 12(2), 11-15.

UN (2020). Recovering better: economic and social challenges and opportunities: A compilation of the High-level Advisory Board on Economic and Social Affairs. New York: United Nations, Department of Economic and Social Affairs. 
UNDP (2008). Strengthening the indigenous drivers of post-conflict economic recovery. In Post-Conflict Economic Recovery: Enabling Local Ingenuity (pp. 48-105). New York: Bureau for Crisis Prevention and Recovery (BCPR), United Nations Development Programme.

Zouhar, Y., Jellema, J., Lustig, N., \& Trabelsi, M. (2021). Public expenditure and inclusive growth: A survey. IMF Working Paper 21/83, Washington, DC: Institute for Capacity Development, International Monetary Fund. 


\section{Appendix}

\section{Economic recovery of Jammu and Kashmir: A study of youth's aspirations and expectations}

\section{Respondent's questionnaire}

Note: You are requested to please express your perceptions regarding economic recovery of Jammu and Kashmir under new regime after abrogation of article 370 .

Thanks in anticipation for your valuable contribution.

\section{Personal information of youth}

Table 1. Personal information of youth

\begin{tabular}{|c|c|c|c|c|c|}
\hline \multicolumn{6}{|c|}{ Personal information of youth } \\
\hline \multicolumn{6}{|l|}{ 1. Respondent name } \\
\hline \multicolumn{6}{|l|}{ 2. District } \\
\hline \multicolumn{6}{|l|}{ 3. Residence } \\
\hline 4. Gender & (a) Female & (b) Male & & & \\
\hline 5. Religion & (a) Hindu & (b) Muslim & (c) Sikh & (d) Christian & (e) Any other \\
\hline 6. Faculty & (a) Arts & (b) Commerce & (c) Science & (d) Law & (e) Any other \\
\hline 7. Age & (a) $20-25$ & (b) $25-30$ & (c) $30-35$ & (d) Above 35 & \\
\hline 8. Educational qualification & (a) Graduate & (b) Post graduate & (c) M. Phil & (d) $\mathrm{Ph} . \mathrm{D}$ & (e) Any other \\
\hline
\end{tabular}

\section{Perceptions of youth on economic recovery}

Table 2. State domestic product

State domestic product

1. Do you know what is State Domestic Product (SDP)?

2. If yes, which sector contributes maximum to the SDP?

3. Compared to other states, your rating of growth of SDP of Jammu and Kashmir (J \& K)?

4. Will there be any improvement in SDP in near future?

5. If yes, then in which priority sector? (a) Yes

(b) No

(a) Agriculture

(b) Service sector

(c) Manufacturing Sector

(d) Any other ( Please mention)

(a) Good

(b) Bad

(a) Yes

(b) No

(a) Agriculture

(b) Service

(c) Manufacturing sector

(d) Any other (Please mention) 
Table 3. Employment

Employment

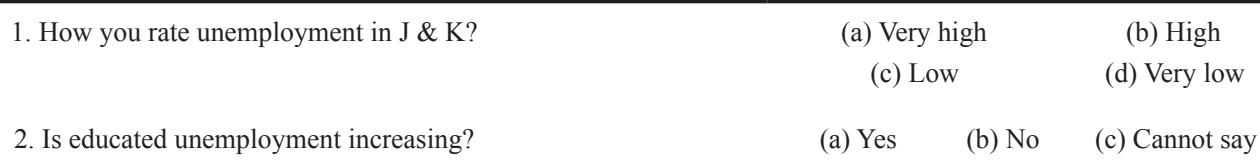

3. If yes, what is the major barrier?

(a) Lack of education

(b) Less aware of job openings

(c) Lack of soft skills

(d) Not enough government jobs

(e) Lack of good jobs

(f) Inadequate access to information

(g) Lack of guidance

(h) Lack of work experience

4. Will the government be able to employ all educated unemployed youth in J \& K?

(a) Yes
(b) No
(c) Cannot say

5. After abrogation of Article 370, job opportunities in the next 5 years will?
(a) Increase
(b) Decrease

(c) Rapidly increase

(d) Rapidly decrease

(e) Cannot say

Table 4. Food security

Food security

1. What is the main source of income in your area?

2. If agriculture, the average size of land holding (ha) $(1 \mathrm{ha}=19.76$ kanal $)$

3. Is the unpredictable and variable nature of climate threat on agricultural productivity?

4. If yes, how it affects the income?

5. What changes you are expecting in food security after abrogation of Article 370 ? (a) Agriculture
(b) Private jobs

(c) Government jobs

(d) Business

(e) Any other (Please mention)

(a) Marginal holdings (up to $1.0 \mathrm{ha}$ )

(b) Small holdings (1-2 ha)

(c) Small-medium holdings (2-4 ha)

(d) Large holdings (Above 10 ha)
(a) Yes
(b) No
(c) Cannot say

(a) Low productivity

(b) Excessive rains

(c) Droughts

(d) Fewer products to sell in the market
(a) Irrigation facilities
(b) Agricultural education
(c) Institutional credit
(d) Transport facilities
(e) Proper marketing facilities
(f) Provision of better manure
(g) Cooperative farming
(h) Supply of quality products
(i) Consolidation of holding
(j) Reduction of population on land 
Table 5. Health

\begin{tabular}{|c|c|}
\hline \multicolumn{2}{|c|}{ Health } \\
\hline 1. Are sufficient health facilities available for people of J \& K? & (c) Cannot say \\
\hline 2. Do you avail any benefit from health facilities in your area? & (a) Yes \\
\hline \multirow[t]{9}{*}{ 3. If yes, the general problems faced by you? } & (a) Expensive \\
\hline & (b) Lack of specialist doctors \\
\hline & (c) Distance problems \\
\hline & (d) Cost of medical service store \\
\hline & (e) Awareness problems \\
\hline & (f) Formalities for registration \\
\hline & (g) Lack of infrastructure \\
\hline & (h) High expenses \\
\hline & (i) Any other (Please mention) \\
\hline 4. Will there be any improvement in health services in near future? & (c) Cannot say \\
\hline
\end{tabular}

Table 6. Educational system

Educational system

1. Are you satisfied with your educational qualification?

2. Having in your mind your current education do you think you can easily find a job?

3. If no, who do you blame the most?

4. The necessary things that must be improved in educational institutions? (a) Yes

(b) No
(a) Self
(b) Your education
(c) Family
(d) Government

(a) Focus more on practical skills

(b) Improve quality of libraries

(c) Modernize educational programmes

(d) Use of modern teaching methods

(e) Modernize content of courses

(f) Better teachers

(g) Better environment

(h) Any other (please mention)

Table 7. Physical infrastructure

\begin{tabular}{|c|c|}
\hline $\begin{array}{l}\text { 1. Are you satisfied with the following facilities in your area? } \\
\text { (Please write Yes/No) }\end{array}$ & $\begin{array}{l}\text { 2. The most necessary things that must be improved in terms of } \\
\text { infrastructure in your area after abrogation of Article } 370 \text { ? }\end{array}$ \\
\hline (b) Power supply & (b) Railways \\
\hline (c) Social environment & (c) Airport infrastructure development \\
\hline (f) Road construction & (f) Highways \\
\hline (g) Education & (g) Renewable energy \\
\hline (h) Health & (h) Health sector \\
\hline (i) Any other (Please mention) & (i) Urban transport \\
\hline
\end{tabular}


Table 8. Marginalized populations

Marginalized populations

1. Is your area has a population of marginalized section?

(a) Yes

(b) No

2. If yes, which section prevails the most?

(a) $\mathrm{SC}$

(b) ST

(c) $\mathrm{OBC}$

(d) Any other

3. Are they discriminative in your area?

(a) Yes

(b) No

4. If yes, then what you think how to overcome?

(a) Avoid segregation

(b) Seek out marginalized choices

(c) Inclusion in our daily life

(d) Support marginalized movements

(e) Any other (Please mention)

Table 9. Banking facilities

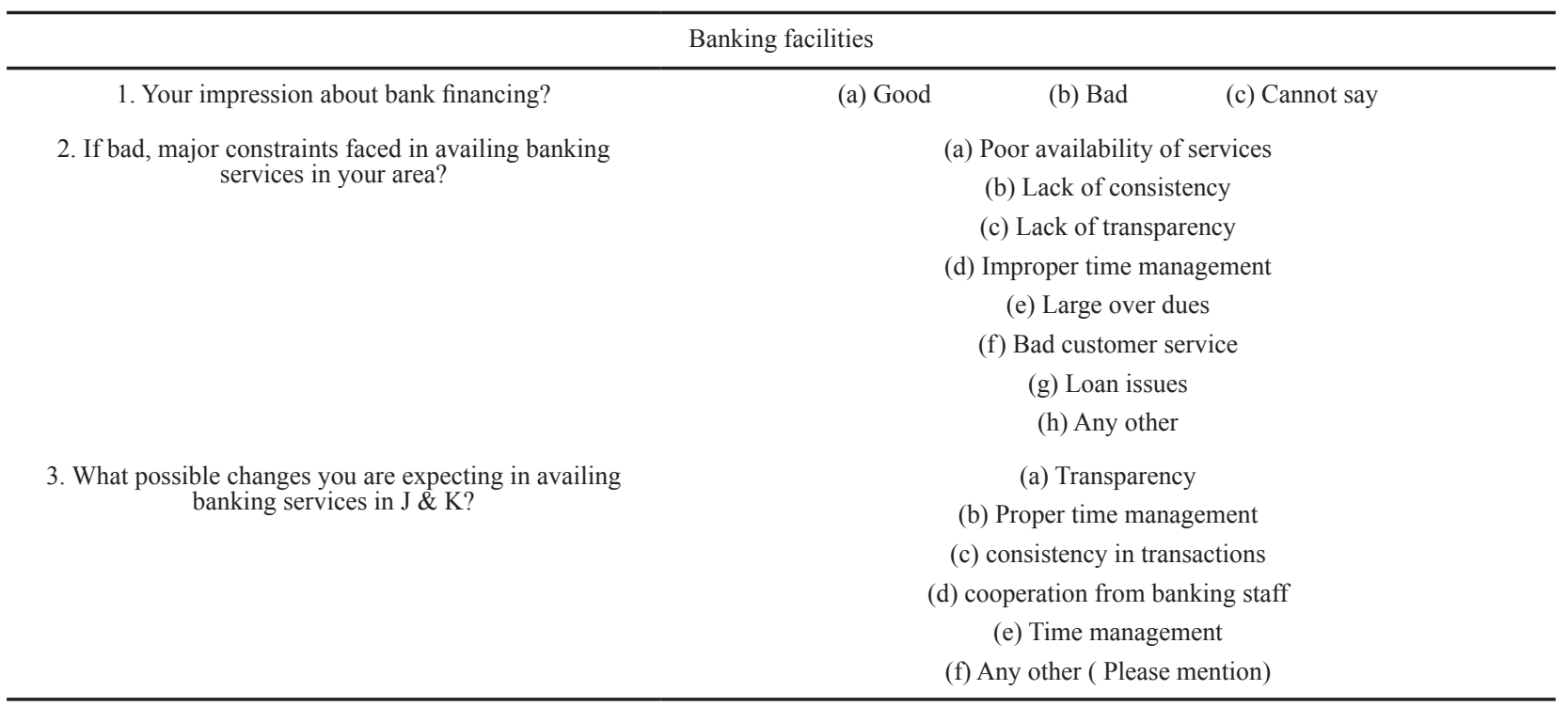

Table 10. Poverty

\section{Poverty}

1. Do you think $\mathrm{J} \& \mathrm{~K}$ is a poor state?

2. If yes, how much it affects you and your family?

3 . Will there be any reduction in poverty in the near future?

$$
\text { 4. If yes, then how? }
$$
(a) Yes
(b) No
(c) Cannot say

(a) Greatly

(b) Moderately

(c) Slightly

(d) Not at all

$\begin{array}{lll}\text { (a) Yes } & \text { (b) No } & \text { (c) Cannot say }\end{array}$
(a) Literacy
(b) Training
(c) Education

(d) Housing facilities

(e) Health facilities

(f) Income and employment

(g) Create jobs

(h) Access to credit

(i) Provide subsidies

(j) Minimum wage laws

(k) Any other (Please mention) 
Table 11. Corruption

\begin{tabular}{|c|c|}
\hline \multicolumn{2}{|c|}{ Corruption } \\
\hline 1. Is J \& $\mathrm{K}$ a corrupt state? & (a) Yes \\
\hline 2. If yes, how it affects the economy? & $\begin{array}{l}\text { (a) Low quality education and health care } \\
\text { (b) Lowers standard of living } \\
\text { (c) Reduces tax revenues } \\
\text { (d) Reduces effectiveness of public investment } \\
\text { (e) Reduces economic performance } \\
\text { (f) Any other (Please mention) }\end{array}$ \\
\hline 3. The ways to reduce corruption in $\mathrm{J} \& \mathrm{~K}$ ? & $\begin{array}{l}\text { (a) Transparency } \\
\text { (b) Empower citizens } \\
\text { (c) Reform finance management } \\
\text { (d) Strong punitive measures } \\
\text { (e) Reducing horizontal inequalities } \\
\text { (f) Any othe (Please mention) }\end{array}$ \\
\hline
\end{tabular}

Table 12. Return of displaced persons

\begin{tabular}{|c|c|c|}
\hline \multicolumn{3}{|c|}{ Return of displaced persons } \\
\hline 1. Do you know the displacement of Kashmiri Pandits? & (a) Yes & (b) No \\
\hline 2. Is their resettlement a good step? & (a) Yes & (b) No \\
\hline 3. What are the ways to resettle them? & \multicolumn{2}{|c|}{$\begin{array}{l}\text { (a) Providing jobs } \\
\text { (b) Providing basic facilities } \\
\text { (c) Free education } \\
\text { (d) By inclusion in the society } \\
\text { (e) Providing separate housing colonies in Kashmir } \\
\text { (f) Any other (Please mention) }\end{array}$} \\
\hline 4. After their resettlement, will there be any affect on societal integration? & (a) Yes & (b) No \\
\hline 5. If yes, what you think it acts as? & (a) Positively & (b) Negatively \\
\hline
\end{tabular}

Table 13. Youth's voilence

\begin{tabular}{|c|c|}
\hline \multicolumn{2}{|c|}{ Youth's voilence } \\
\hline 1. Are youth of $\mathrm{J} \& \mathrm{~K}$ involved in violence? & (c) Cannot say \\
\hline 2. If yes, what are the reasons of their involvement? & $\begin{array}{l}\text { (a) Lack of jobs } \\
\text { (b) Unequal access to relief } \\
\text { (c) Unequal access to development assistance } \\
\text { (d) Exclusion in education } \\
\text { (e) Exclusion in employment } \\
\text { (f) Unequal access to social services } \\
\text { (g) Widespread corruption } \\
\text { (h) Restriction on cultural and social practices } \\
\text { (i) Economic insecurity } \\
\text { (j) Any other (please mention) }\end{array}$ \\
\hline $\begin{array}{l}\text { 3. Will there be cessation of hostilities and violence of youth } \\
\text { after removal of Article } 370 \text { ? }\end{array}$ & $\begin{array}{ll}\text { (b) No } \quad \text { (c) Cannot say }\end{array}$ \\
\hline 4. If yes, then how? & $\begin{array}{l}\text { (a) By providing employment opportunities } \\
\text { (b) Need to address the issues of corruption } \\
\text { (c) Neutral and fair media } \\
\text { (d) Established well governed political institution } \\
\text { (e) Inclusion in education } \\
\text { (f) Economic security } \\
\text { (g) By providing skill development programmes } \\
\text { (h) Any other (please mention) }\end{array}$ \\
\hline
\end{tabular}


Table 14. Youth's training

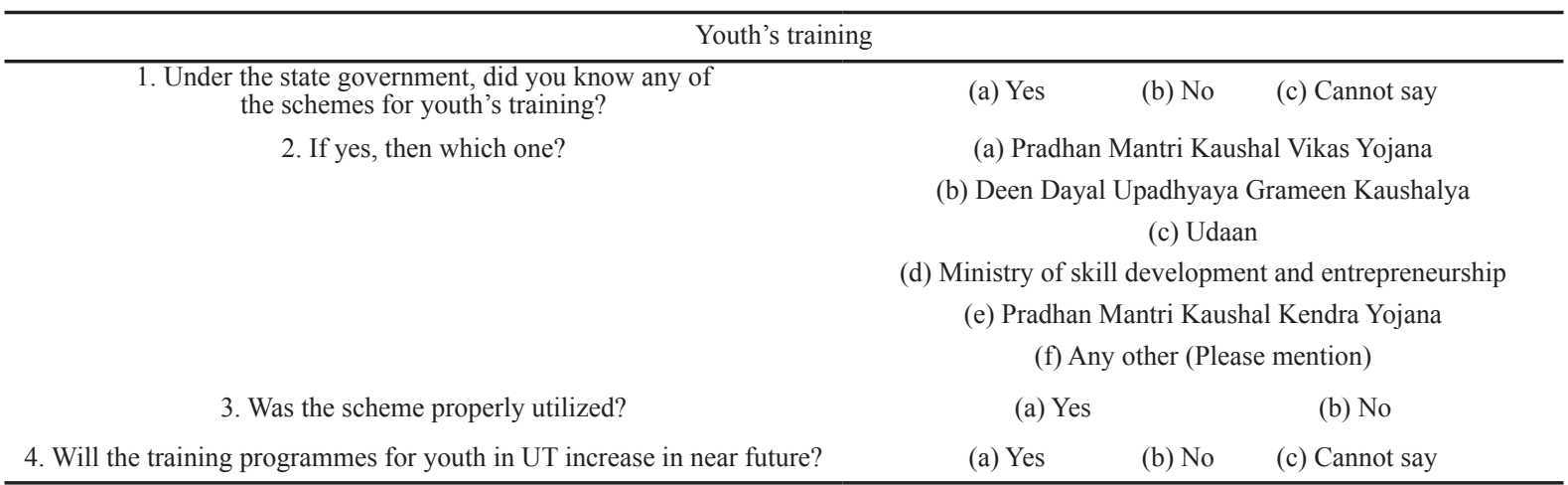

Table 15. Self employment echemes

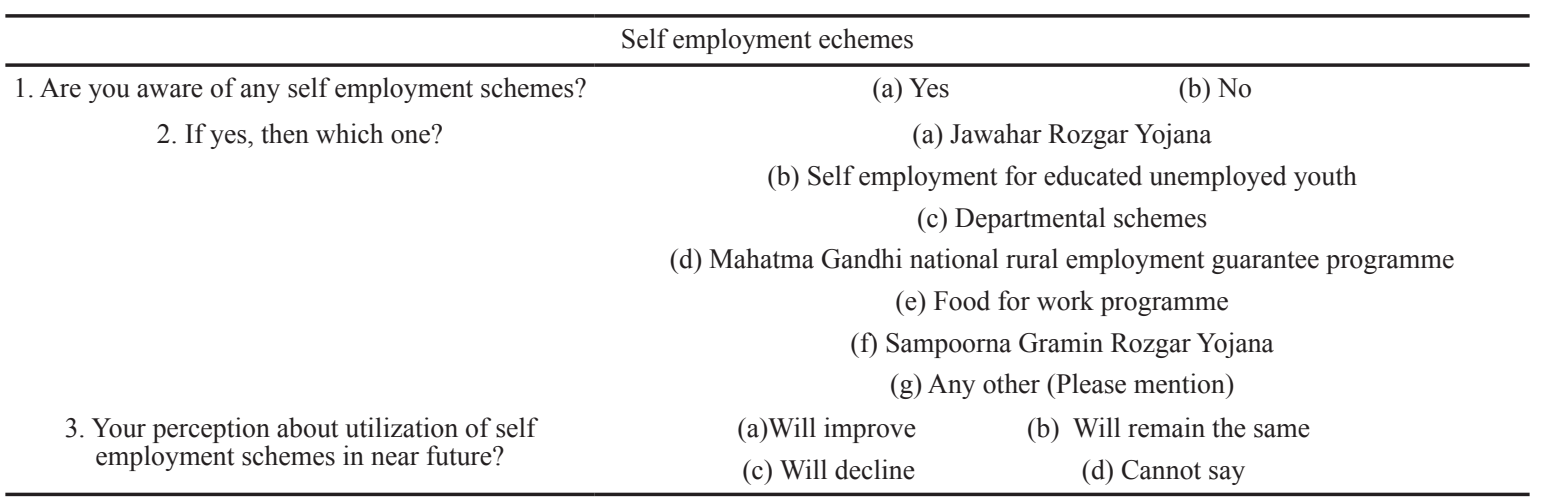

Table 16. Tourism

\begin{tabular}{|c|c|}
\hline & Tourism \\
\hline 1. Is there any scope of tourism in your area? & (a) Yes \\
\hline 2. If yes, on which ground is based on? & (b) Manmade resources \\
\hline 3. Will the Tourism industry flourished in the coming years? & (a) Same $\quad$ (b) Improve \\
\hline 4. How tourism contribute to life in your area? & $\begin{array}{c}\text { (a) Created more jobs } \\
\text { (b) Attract more investment } \\
\text { (c) Provide more business for local people } \\
\text { (d) Support museums and heritage site } \\
\text { (e) Diversified households/community livelihood choices and opportunities } \\
\text { (f) Generate more income } \\
\text { (g) Improvement in the standard of living } \\
\text { (h) Foreign exchange earnings } \\
\text { (i) Any other (Please mention) }\end{array}$ \\
\hline 5. Roles the government should play for development of tourism? & $\begin{array}{l}\text { (a) Good infrastructure } \\
\text { (b) Availability of transport facilities } \\
\text { (c) Formulate tourism policies } \\
\text { (d) Financial investment } \\
\text { (e)Transport facility } \\
\text { (f) Parking facility } \\
\text { (g) Bank (ATM) facility } \\
\text { (h) Super market } \\
\text { (i) Restaurant } \\
\text { (j) Any other (Please mention) }\end{array}$ \\
\hline
\end{tabular}


Table 17. Horizontal inequalities

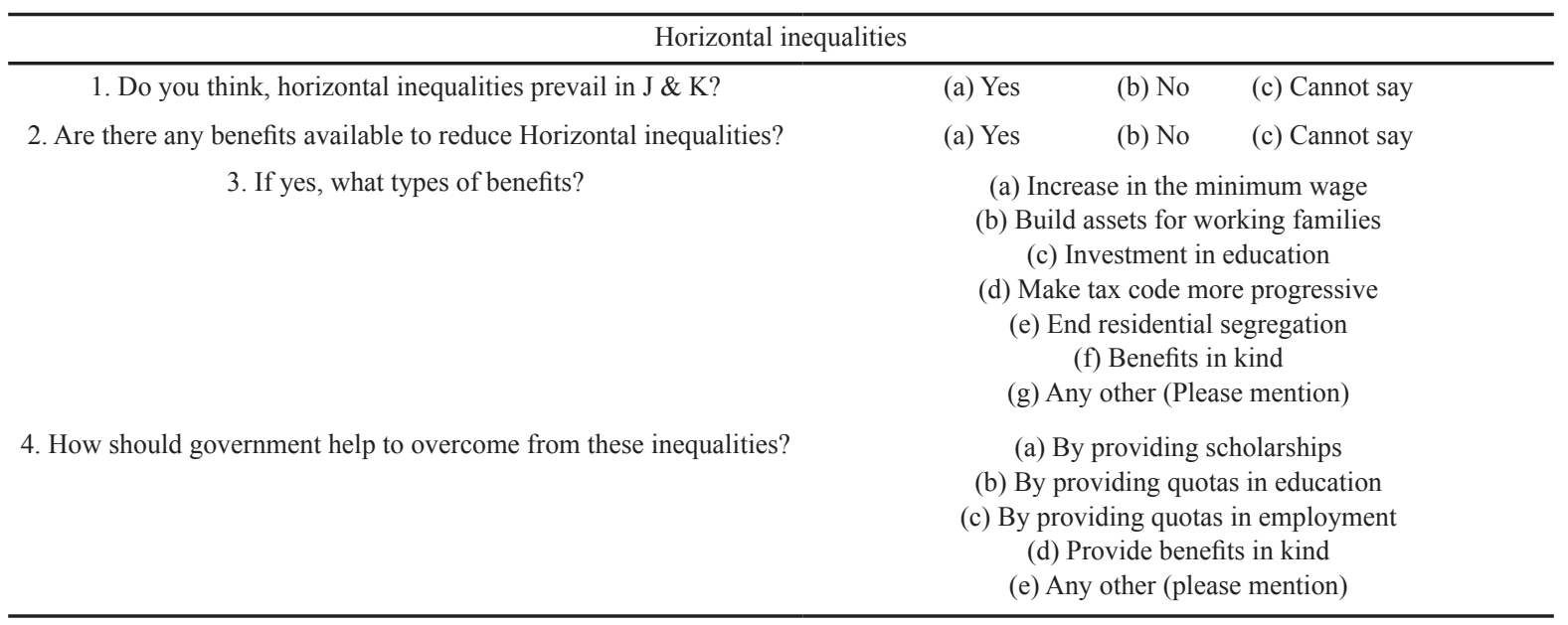

Table 18. Horticulture

\begin{tabular}{|c|c|}
\hline \multicolumn{2}{|c|}{ Horticulture } \\
\hline 1. Is horticulture, a backbone of J \& K economy? & (c) Cannot say \\
\hline 2. If yes, then how? & $\begin{array}{l}\text { (a) Raise income level } \\
\text { (b) Generate employment } \\
\text { (c) Alleviate poverty } \\
\text { (d) Export promotion } \\
\text { (e) Provides food and nutritional secure } \\
\text { (f) Maintain ecological balance } \\
\text { (g) Provides raw materials } \\
\text { (h) Any other (Please mention) }\end{array}$ \\
\hline $\begin{array}{l}\text { 3. On what issues government should focus to further } \\
\text { boost this sector? RANK (1-6) }\end{array}$ & $\begin{array}{c}\text { (a) Transportation } \\
\text { (b) Storage facilities } \\
\text { (c) Skilled labour } \\
\text { (d) Market strategy } \\
\text { (e) Finance } \\
\text { (f) Any other (Please mention) }\end{array}$ \\
\hline
\end{tabular}

Table 19. Handicrafts

\begin{tabular}{|c|c|}
\hline \multicolumn{2}{|c|}{ Handicrafts } \\
\hline 1. Is handicraft, a backbone of $\mathrm{J} \& \mathrm{~K}$ economy? & (c) Cannot say \\
\hline 2. If yes, how much it contributes to SDP? & (b) Moderate \\
\hline 3. The problems handicrafts sector faced? & $\begin{array}{c}\text { (a) Unorganized structure } \\
\text { (b) Lack of skilled education } \\
\text { (c) Low capital } \\
\text { (d) Poor Infrastructure } \\
\text { (e) Absence of market intelligence) } \\
\text { (f) Any other (Please mention) }\end{array}$ \\
\hline 4. How the performance of this sector will improve? & $\begin{array}{l}\text { (a) Product marketing } \\
\text { (b) Financial assistance } \\
\text { (c) Interest free loan } \\
\text { (d) Establish clusters of Handicrafts } \\
\text { (e) Skilled labour } \\
\text { (f) Any other (Please mention) }\end{array}$ \\
\hline
\end{tabular}


Table 20. Start of economic recovery

Start of economic recovery

1. Which is the best way to start economic recovery in $\mathrm{J} \& \mathrm{~K}$ ?

2. Is central government mainly responsible for it?

3. If yes, then how?

5. Which field needs more attention?
4. Will there be recovery in economic activities in near future?

(a) Revenue mobilization

(b) Trade

(c) Investment

(d) People participation

(e) Reduction in corruption
(f) Transparency
(g) Infrastructure

(h) Power development
(i) Agriculture
(j) Handicrafts
(k) Horticulture
(1) Any other
(a) Yes
(b) No

(a) Proper policy and planning

(b) By raising state revenues

(c) By policy reforms

(d) Economic management

(e) Government expenditure

(f) Policy reforms

(g) Fostering private investmen

(h) Any other (please mention)

(a) Yes

(b) No

(c) Cannot say

(a)Health

(b) Education

(c) Trade

(d) Banking

(e) Agriculture

(f) Industry

(g) Transport

(h) Power

(i) Handicrafts

(j) Tourism

(k) Renewable energy

(1) Any other (please mention)

Table 21. Participation of local people in the development

Participation of local people in the development

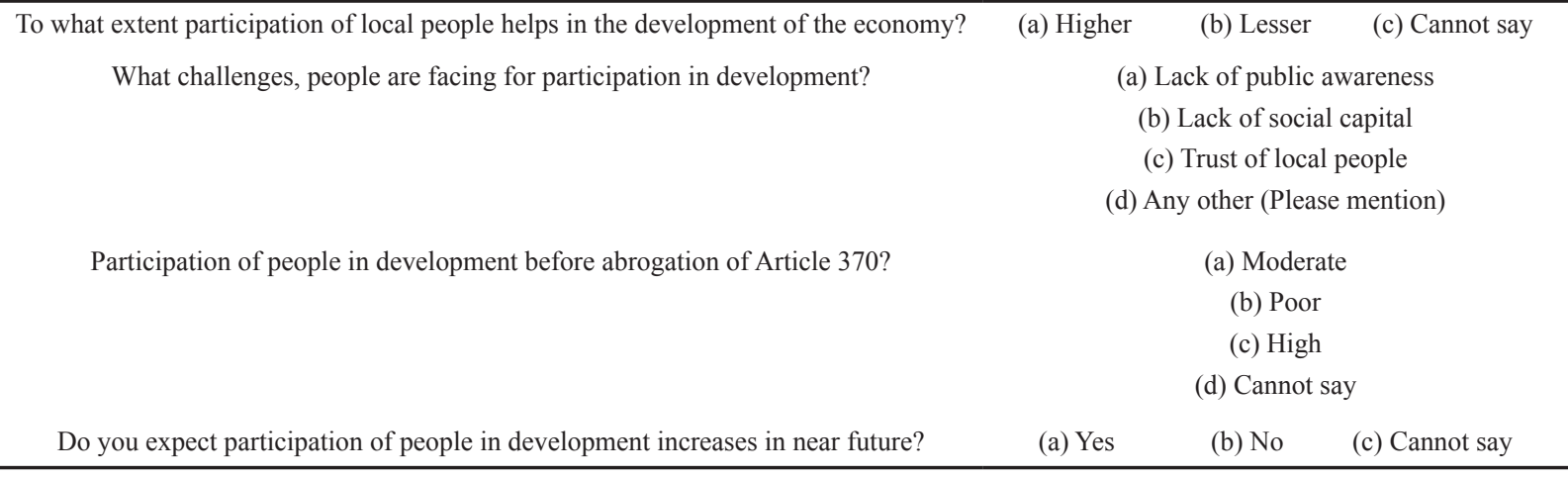


Table 22. Investment

\section{Investment}

1. Do you think the investment opportunity will foster after abrogation of Article 370 ?

(a) Yes

(b) No

(c) Cannot say

2 .if yes, what do you expect which field gets higher preference?

(a) Tourism

(b) Private business

(c) Roads

(d) Higher education institutions

(e) Railways

(f) Hospitals

(g) Increase in the number of hospitals

(h) Agriculture

(i) Any other (Please mention)

Table 23. Macroeconomic policies

Macroeconomic policies

1. In the regime of Article 370, what do you think about the macroeconomic policy of government?

(a) Good

(b) $\mathrm{Bad}$

(c) Cannot say

2. If good, did the benefits reach the public?

3. Do you think, the policy designed and its implementation will benefits the people after abrogation of Article 370?

4. If yes, government should focused on what types of policies to increase the economic growth? (a) Yes

(b) No

(c) Cannot say

(a) Yes

(b) No

(a) Increase government spending

(b) Cutting taxes

(c) Roads

(d) Tourism

(e) Improved education

(f) Agriculture

(g) Improved Infrastructure

(h) Power development

(i) Privatization

(j) Education

(k) Health

(1) Water

(m) Energy

(n) Employment

(o) Self employment

(p) Any other (Please mention) 